\title{
BIFURCATION OF A UNIQUE STABLE PERIODIC ORBIT FROM A HOMOCLINIC ORBIT IN INFINITE-DIMENSIONAL SYSTEMS
}

\author{
SHUI-NEE CHOW AND BO DENG
}

\begin{abstract}
Under some generic conditions, we show how a unique stable periodic orbit can bifurcate from a homoclinic orbit for semilinear parabolic equations and retarded functional differential equations. This is a generalization of a result of Šil'nikov for ordinary differential equations.
\end{abstract}

\section{INTRODUCTION}

Consider an autonomous differential equation in the plane with a real parameter $\varepsilon$ :

$$
\dot{x}=f(x, \varepsilon),
$$

where $x \in \mathbf{R}^{2}$. Assume that $x=0$ is a hyperbolic equilibrium for all small $\varepsilon$ and $D_{x} f(0, \varepsilon)=A$. Suppose that $\lambda>0$ and $\mu<0$ are the eigenvalues of $A$ and

$$
\lambda+\mu<0 .
$$

Assume that there exists a homoclinic orbit of $(0.1)$ to the origin 0 at $\varepsilon=0$. It is well known that under certain transversality conditions on equation $(0.1)$ an exponentially stable periodic orbit will bifurcate from the homoclinic orbit as the parameter $\varepsilon$ changes (see Andronov, Leontovich, Gordon and Maier [1] and Chow and Hale [3], for example, for more details). In [9] Neimark and Šil'nikov generalized the above result to $\mathbf{R}^{3}$ replacing condition $(0.2)$ by an appropriate condition on the eigenvalues. This generalization is nontrivial since we are not able to make a smooth change of coordinates to reduce the study of local behavior of solutions near a hyperbolic equilibrium to that of a linear system. In fact, all the difficulties are related to some fine estimates on the longtime behavior of solutions in a small neighborhood of a hyperbolic equilibrium. In [10], Šil'nikov considered similar problems in higher dimensions. (For some interesting applications, see, for example, Evans, Fenichel and Feroe [4] and Feroe [5].)

Received by the editors June 5, 1986 and, in revised form, June 8, 1987 and October 13, 1987.

1980 Mathematics Subject Classification (1985 Revision). Primary 35K22, 34K99; Secondary 35B33, 35B10.

The first author was partially supported by DARPA and NSF Grant DMS 8401719 . 
The purpose of this paper is to show that Šil'nikov's theorem may be generalized to some infinite-dimensional systems. We consider two kinds of infinitedimensional systems, namely, semilinear parabolic differential equations and retarded functional differential equations. Since the flows defined by these equations are semiflows (solutions may not be extended backwards in time), we are not able to use Šil' nikov's method directly for our problems. However, the basic approach is based on an idea of Šil'nikov [10] which reduces the bifurcation problem to continuation of fixed points for a one-parameter family of maps (see (iii) in $\S 2$ for semilinear parabolic equations or Lemma 3.11 in $\S 3$ for retarded functional differential equations). As in the finite-dimensional cases, the main difficulty in the proof is to obtain local estimates of solutions of our equations near hyperbolic equilibria. We note that in many aspects, our estimates are new even in the finite-dimensional cases. Our estimates are all related to linear variational equations along semiorbits of the nonlinear equations. By using these estimates, we are able to obtain smoothness properties of the above mentioned maps and to find the fixed points.

In [2 and 12], similar results are obtained by using different methods. In [2], Blazqueg generalized Šil'nikov's theorem to semilinear parabolic equations by using Šil' nikov's work [10] on finite-dimensional systems. This work seems to be sketchy. In [12], Walther generalized Šil' nikov's theorem to retarded functional differential equations. Walther's approach is based on the $\lambda$-lemma (inclination lemma) for retarded functional differential equations.

We also note that while we require the vector fields to be at least $C^{3}$, Walther needs only $C^{2}$ vector fields and obtains similar results for functional differential equations.

In $\S 1$, we consider semilinear parabolic equations. We derive all the necessary estimates required in the proof of our main result. In $\S 2$, we construct the oneparameter family of maps and prove our main result (Theorem 2.3). Retarded functional differential equations are treated in $\S 3$. An application is given in $\S 4$. In this paper, we will give detailed proofs for semilinear parabolic equations. For retarded functional differential equations, we will only give proofs when they are different from those for semilinear parabolic equations.

Acknowledgment. We thank the referee of this paper for many very helpful improvements. This paper has been substantially revised from its first version. Most of the improvements are due to the careful reading of the referee.

\section{LoCAL ANALYSIS}

In this section, we consider the behavior of solutions of a semilinear equation in a Banach space near a hyperbolic equilibrium. Our estimates are based on the work of Henry [8] and a modified Gronwall's inequality (Lemma 1.1). The main estimate is stated in Lemma 1.3 which gives the exponential bounds in finite time of solutions of the variational equation along a solution in a small neighborhood of the equilibrium. We also give the upper and lower bound for 
the time for which a solution will stay in a fixed neighborhood of the hyperbolic equilibrium. These estimates seem to be new even for ordinary differential equations in $\mathbf{R}^{d}$. The main purpose of this section is to define a map in a small neighborhood of the hyperbolic equilibrium which is closely related to the usual Poincaré map but is somewhat different. In Lemma 1.7, we show that this map is Lipschitz with a small Lipschitz constant. The construction of the map is based on Sil'nikov's ideas [10] and the smoothness is based on the estimates.

Let $X$ be a Banach space with norm $|\cdot|$ and $A: X \rightarrow X$ be a linear sectoral operator with dense domain $\mathscr{D}(A)$ which generates an analytic semigroup $\left\{e^{-A t}, t \geq 0\right\}$ on $X$. For $0 \leq \alpha<1$, let $A^{\alpha}$ be the $\alpha$-fractional power of $A$ (see Henry [8, pp. 24-30]) with domain $\mathscr{D}\left(A^{\alpha}\right)$. Let $\sigma(A)$ be the spectrum of $A$ and assume that $\operatorname{Re} \sigma(A)>0$. Define for each $0 \leq \alpha<1$,

$$
X^{\alpha}=\mathscr{D}\left(A^{\alpha}\right), \quad|x|_{\alpha}=\left|A^{\alpha} x\right| .
$$

It is well known that $X^{\alpha}$ is a Banach space with norm $|\cdot|_{\alpha}$ and $X^{0}=X$. Furthermore, for $\alpha \geq \beta \geq 0, X^{\alpha}$ is a dense subspace of $X^{\beta}$ with continuous inclusion. Let $Y_{1}$ and $Y_{2}$ be Banach spaces. We denote by $L\left(Y_{1}, Y_{2}\right)$ the Banach space of all bounded linear operators from $Y_{1}$ to $Y_{2}$ equipped with the operator norm induced by the norms of $Y_{1}$ and $Y_{2}$.

Let $U_{0} \subset X^{\alpha} \times \mathbf{R}$ be an open neighborhood of the origin and $\varepsilon_{0}>0$ be fixed. Consider the following semilinear autonomous equation with a parameter $\varepsilon \in\left[-\varepsilon_{0}, \varepsilon_{0}\right]$ :

$$
\begin{aligned}
& \dot{x}=-A x+f(x, y, \varepsilon), \quad(x, y) \in U_{0}, \varepsilon \in\left[-\varepsilon_{0}, \varepsilon_{0}\right], \\
& \dot{y}=\lambda y+g(x, y, \varepsilon), \quad(x, y) \in U_{0}, \varepsilon \in\left[-\varepsilon_{0}, \varepsilon_{0}\right],
\end{aligned}
$$

where $f: U_{0} \times\left[-\varepsilon_{0}, \varepsilon_{0}\right] \rightarrow X, g: U_{0} \times\left[-\varepsilon_{0}, \varepsilon_{0}\right] \rightarrow \mathbf{R}$ and $\lambda>0$ is fixed.

Let $\sigma(A)$ be the spectrum of $A$. Consider the following hypotheses:

(H1) $\operatorname{Re} \sigma(A) \geq \mu>\lambda>0$, where $\mu$ is a fixed constant;

(H2) $f \in C^{3}\left[U_{0} \times\left[-\varepsilon_{0}, \varepsilon_{0}\right], X\right], g \in C^{3}\left[U_{0} \times\left[-\varepsilon_{0}, \varepsilon_{0}\right], \mathbf{R}\right]$ and

$$
\begin{array}{lll}
f(0,0, \varepsilon)=0, & D_{(x, y)} f(0,0,0)=0, & \varepsilon \in\left[-\varepsilon_{0}, \varepsilon_{0}\right], \\
g(0,0, \varepsilon)=0, & D_{(x, y)} g(0,0,0)=0, & \varepsilon \in\left[-\varepsilon_{0}, \varepsilon_{0}\right],
\end{array}
$$

where the derivatives are taken in the Banach space $X^{\alpha} \times \mathbf{R}$.

By hypotheses (H1) and (H2), there exists a neighborhood $B\left(\delta_{1}\right)=\{(x, y)$ : $\left.|x|_{\alpha}<\delta_{1},|y|<\delta_{1}\right\}$ of $(0,0)$ contained in $U_{0}$ and $0<\varepsilon_{1}<\varepsilon_{0}$ such that the local stable and unstable manifolds $W_{\mathrm{loc}}^{s}(\varepsilon)$ and $W_{\mathrm{loc}}^{u}(\varepsilon)$ exist in $B\left(\delta_{1}\right)$ for $\varepsilon \in\left[-\varepsilon_{1}, \varepsilon_{1}\right]$, where $\delta_{1}>0$ is a small constant. Furthermore they are $C^{3}$-manifolds and are given by

$$
\begin{aligned}
& W_{\mathrm{loc}}^{s}(\varepsilon)=\left\{(x, y) \in B\left(\delta_{1}\right): y=h^{s}(x, \varepsilon),|x|_{\alpha}<\delta_{1}\right\}, \\
& W_{\mathrm{loc}}^{u}(\varepsilon)=\left\{(x, y) \in B\left(\delta_{1}\right): x=h^{u}(y, \varepsilon),|y|<\delta_{1}\right\},
\end{aligned}
$$


where $h^{s}$ and $h^{u}$ are $C^{3}$ in their arguments satisfying

$$
\begin{gathered}
h^{s}(0, \varepsilon)=0, \quad h^{u}(0, \varepsilon)=0, \quad \varepsilon \in\left[-\varepsilon_{1}, \varepsilon_{1}\right], \\
D_{x} h^{s}(0,0)=0, \quad D_{y} h^{u}(0,0)=0 .
\end{gathered}
$$

(See Henry [8, pp . 112-116].)

For $\varepsilon \in\left[-\varepsilon_{1}, \varepsilon_{1}\right]$ we define a diffeomorphism $H(\cdot, \cdot, \varepsilon)$ by

$$
(\bar{x}, \bar{y})=H(x, y, \varepsilon)=\left(x-h^{u}(y, \varepsilon), y\right)
$$

which is near the identity map by (1.5). Its inverse map is

$$
(x, y)=H^{-1}(\bar{x}, \bar{y}, \varepsilon)=\left(\bar{x}+h^{u}(\bar{y}, \varepsilon), \bar{y}\right), \quad \varepsilon \in\left[-\varepsilon_{1}, \varepsilon_{1}\right] .
$$

Let $(x(t), y(t))$ be a classical solution of (1.1) (see Henry [8, pp. 53-55]) and $\bar{U}_{1}(\varepsilon)=H\left(B\left(\delta_{1}\right), \varepsilon\right)$. Since $y(t)$ is finite dimensional, $H$ maps the solution $(x(t), y(t))$ of $(1.1)$ into the solution $(\bar{x}(t), \bar{y}(t))=H(x(t), y(t), \varepsilon)$ of

$$
\begin{array}{ll}
\dot{\bar{x}}=A \bar{x}+\bar{f}(\bar{x}, \bar{y}, \varepsilon), \quad & (\bar{x}, \bar{y}) \in \bar{U}_{1}(\varepsilon), \varepsilon \in\left[-\varepsilon_{1}, \varepsilon_{1}\right], \\
\dot{y}=\lambda \bar{y}+\bar{g}(\bar{x}, \bar{y}, \varepsilon), & (\bar{x}, \bar{y}) \in \bar{U}_{1}(\varepsilon), \varepsilon \in\left[-\varepsilon_{1}, \varepsilon_{1}\right],
\end{array}
$$

where

$$
\begin{aligned}
\bar{f}(\bar{x}, \bar{y}, \varepsilon)= & f\left(H^{-1}(\bar{x}, \bar{y}, \varepsilon), \varepsilon\right)+A h^{u}(\bar{y}, \varepsilon) \\
& -D_{\bar{y}} h^{u}(\bar{y}, \varepsilon)\left[\lambda \bar{y}+g\left(H^{-1}(\bar{x}, \bar{y}, \varepsilon), \varepsilon\right)\right], \\
\bar{g}(\bar{x}, \bar{y}, \varepsilon)= & g\left(H^{-1}(\bar{x}, \bar{y}, \varepsilon), \varepsilon\right) .
\end{aligned}
$$

Conversely, let $(\bar{x}(t), \bar{y}(t))$ be a solution of (1.8). Note that $\bar{y}(t)$ is finite dimensional. By (1.7), $(x(t), y(t))=H^{-1}(\bar{x}(t), \bar{y}(t), \varepsilon)$ is a solution of $(1.1)$. Thus, we have shown that equation (1.1) is conjugate to equation (1.8) via the change of variables (1.6).

Since $H(\cdot, \cdot, \varepsilon)$ is near the identity map for all $\varepsilon \in\left[-\varepsilon_{1}, \varepsilon_{1}\right]$, without loss of generality we assume there is a neighborhood $\bar{U}_{1}$ of $(0,0)$ such that $\bar{U}_{1} \subset \bar{U}_{1}(\varepsilon)$ for every $\varepsilon \in\left[-\varepsilon_{1}, \varepsilon_{1}\right]$. Let $\delta_{2}>0$ be so small that $B\left(\delta_{2}\right)=$ $\left\{(\bar{x}, \bar{y}):|\bar{x}|_{\alpha}<\delta_{2},|y|<\delta_{2}\right\} \subset \bar{U}_{1}$.

Note that (1.9) implies that $\bar{f} \in C^{2}\left[B\left(\delta_{2}\right) \times\left[-\varepsilon_{1}, \varepsilon_{1}\right], X\right]$. By the definition of $H,\left\{(\bar{x}, \bar{y}): \bar{x}=0,(\bar{x}, \bar{y}) \in \bar{U}_{1}\right\}$ is the local invariant unstable manifold of equation $(1.8)$ for all $\varepsilon \in\left[-\varepsilon_{1}, \varepsilon_{1}\right]$. Hence $\bar{f}(0, \bar{y}, \varepsilon)=0$ for $(0, \bar{y}) \in \bar{U}_{1}$ and $\varepsilon \in\left[-\varepsilon_{1}, \varepsilon_{1}\right]$. Therefore, we have

$$
\bar{f}(\bar{x}, \bar{y}, \varepsilon)=\bar{P}(\bar{x}, \bar{y}, \varepsilon) \cdot \bar{x},
$$

where

$$
\bar{P}(\bar{x}, \bar{y}, \varepsilon)=\int_{0}^{1} D_{\bar{x}} \bar{f}(s \bar{x}, \bar{y}, \varepsilon) d s: B\left(\delta_{2}\right) \times\left[-\varepsilon_{1}, \varepsilon_{1}\right] \rightarrow L\left(X^{\alpha}, X\right) .
$$

Note that $\bar{P}$ is $C^{1}$ since $\bar{f}$ is $C^{2}$. 
It follows that by an appropriate change of variables we may assume that the local unstable manifold of equation (1.1) is given by the $y$-axis in the neighborhood $U_{1}$ of $(0,0)$ and

$$
f(x, y, \varepsilon)=P(x, y, \varepsilon) \cdot x
$$

However, we will lose two derivatives in this process. This says that if we assume $f$ has the form (1.10), then the function $P$ is only $C^{1}$.

In the following, we assume $f$ takes the form (1.10), $P$ is $C^{1}$ and $U_{1}=$ $B\left(\delta_{2}\right)$. By hypothesis (H2) we choose $\delta_{2}>0,0<\varepsilon_{2}<\varepsilon_{1}$ and a constant $C_{1}>0$ so that for every $(x, y, \varepsilon) \in B\left(\delta_{2}\right) \times\left[-\varepsilon_{2}, \varepsilon_{2}\right]$, we have

$$
\begin{gathered}
\left|D_{x} h^{s}(x, \varepsilon)\right| \leq C_{1}|x|_{\alpha}, \\
\left|D_{y} h^{u}(y, \varepsilon)\right|_{\alpha} \leq C_{1}|y|, \\
|P(x, y, \varepsilon)|_{\alpha} \leq C_{1}\left(|x|_{\alpha}+|y|\right), \\
\left|D_{(x, y)} P(x, y, \varepsilon)\right|_{\alpha} \leq C_{1}, \\
|g(x, y, \varepsilon)| \leq C_{1}\left(|x|_{\alpha}+|y|\right)^{2}, \\
\left|D_{(x, y)} g(x, y, \varepsilon)\right| \leq C_{1}\left(|x|_{\alpha}+|y|\right) .
\end{gathered}
$$

For each $(\xi, \eta, \varepsilon) \in B\left(\delta_{2}\right) \times\left[-\varepsilon_{2}, \varepsilon_{2}\right]$, there exists a unique classical solution $(x(t ; \xi, \eta, \varepsilon), y(t ; \xi, \eta, \varepsilon)) \in B\left(\delta_{2}\right), 0 \leq t<T \leq+\infty$, for some $T$ which depends on $(\xi, \eta, \varepsilon)$ satisfying the variation of constants formula

$$
\begin{aligned}
& x(t ; \xi, \eta, \varepsilon)=e^{-A t} \xi+\int_{0}^{t} e^{-A(t-s)} f(x(s ; \xi, \eta, \varepsilon), y(s ; \xi, \eta, \varepsilon), \varepsilon) d s \\
& y(t ; \xi, \eta, \varepsilon)=e^{\lambda t} \eta+\int_{0}^{t} e^{\lambda(t-s)} g(x(s ; \xi, \eta, \varepsilon), y(s ; \xi, \eta, \varepsilon), \varepsilon) d s
\end{aligned}
$$

Furthermore, for fixed $t, x(t ; \cdot, \cdot, \cdot): B\left(\delta_{2}\right) \times\left[-\varepsilon_{2}, \varepsilon_{2}\right] \rightarrow X^{\alpha}$ and $y(t ; \cdot, \cdot, \cdot)$ : $B\left(\delta_{2}\right) \times\left[-\varepsilon_{2}, \varepsilon_{2}\right] \rightarrow \mathbf{R}$ are $C^{3}$ and the derivatives $p(t)=D_{(\xi, \eta)} x(t ; \xi, \eta, \varepsilon)$ and $q(t)=D_{(\xi, \eta)} y(t ; \xi, \eta, \varepsilon)$ are the unique solutions of the integral equations

$$
\begin{aligned}
& p(t)=e^{-A t} p(0)+\int_{0}^{t} e^{-A(t-s)}\left[D_{x} f(x(s ; \xi, \eta, \varepsilon), y(s ; \xi, \eta, \varepsilon), \varepsilon) p(s)\right. \\
& \left.+D_{y} f(x(s ; \xi, \eta, \varepsilon), y(s ; \xi, \eta, \varepsilon), \varepsilon) q(s)\right] d s, \\
& q(t)=e^{\lambda t} q(0)+\int_{0}^{t} e^{\lambda(t-s)}\left[D_{x} g(x(s ; \xi, \eta, \varepsilon), y(s ; \xi, \eta, \varepsilon), \varepsilon) p(s)\right. \\
& \left.+D_{y} g(x(s ; \xi, \eta, \varepsilon), y(s ; \xi, \eta, \varepsilon), \varepsilon) q(s)\right] d s
\end{aligned}
$$

for $0 \leq t<T$. Note that $(p(t), q(t)) \in L\left(X^{\alpha} \times \mathbf{R}, X^{\alpha} \times \mathbf{R}\right)$ and $(p(0), q(0))=$ $I: X^{\alpha} \times \mathbf{R} \rightarrow X^{\alpha} \times \mathbf{R}$ (see Henry [8, pp. 62-65]). 
Under the assumption on the operator $A$ and its spectrum $\sigma(A)$ we may assume the norm $|\cdot|$ on $X^{0}=X$ has been chosen so that

$$
\begin{aligned}
& \left|e^{-A t} x\right|_{\alpha} \leq e^{-\mu t}|x|_{\alpha}, \quad t \geq 0, \\
& \left|e^{-A t} x\right|_{\alpha} \leq \frac{C_{\alpha}}{t^{\alpha}} e^{-\mu t}|x|, \quad t>0,
\end{aligned}
$$

where $C_{\alpha}$ is a constant depending on $\alpha$ (see Henry [8, p. 31]).

To obtain growth estimates for solutions in $B\left(\delta_{2}\right) \times\left[-\varepsilon_{2}, \varepsilon_{2}\right]$, we need the following version of Gronwall's inequalities:

Lemma 1.1. Let $u, a:[0, \infty) \rightarrow[0, \infty)$ be locally integrable, and $b$ be a positive constant. If $0 \leq \alpha<1$ and

$$
u(t) \leq a(t)+b \int_{0}^{t} \frac{1}{(t-s)^{\alpha}} u(s) d s, \quad 0 \leq t<\infty,
$$

then

$$
u(t) \leq a(t)+[b \Gamma(1-\alpha)]^{1 / 1-\alpha} \int_{0}^{t} E_{\alpha}^{\prime}\left((t-s)[b \Gamma(1-\alpha)]^{1 / 1-\alpha}\right) a(s) d s,
$$

where

$$
[b \Gamma(1-\alpha)]^{1 / 1-\alpha}>0, \quad E_{\alpha}(t)=\sum_{n=0}^{\infty} t^{n(1-\alpha)} / \Gamma(n(1-\alpha)+1)
$$

and $\Gamma$ is the usual Gamma function.

Proof. See Henry [8, pp. 188-189].

Remarks. (1) $E_{\alpha}^{\prime}(t) \cdot t^{\alpha} \Gamma(1-\alpha)$ is bounded as $t \rightarrow 0^{+}$. Both $E_{\alpha}^{\prime}(t) \cdot(1-\alpha) e^{-t}$ and $E_{\alpha}(t) \cdot(1-\alpha) e^{-t}$ are bounded as $t \rightarrow+\infty$, which implies $E_{\alpha}(t)(1-\alpha) e^{-t}$ is bounded on $[0,+\infty)$.

(2) If $a(t) \equiv a$, a constant, then

$$
u(t) \leq a\left(1+E_{\alpha}\left(t[b \Gamma(1-\alpha)]^{1 / 1-\alpha}\right)\right) .
$$

Lemma 1.2. Let $T>0$ be fixed and $\varepsilon \in\left[-\varepsilon_{2}, \varepsilon_{2}\right]$. Suppose

$$
(x(t ; \xi, \eta, \varepsilon), y(t ; \xi, \eta, \varepsilon)) \in B\left(\delta_{2}\right)
$$

for all $0 \leq t \leq T$. Then

$$
|x(t ; \xi, \eta, \varepsilon)|_{\alpha} \leq|\xi|_{\alpha}\left(1+E_{\alpha}\left(\theta\left(\delta_{2}\right) t\right)\right) e^{-\mu t}, \quad 0 \leq t \leq T,
$$

where $\theta(\delta)=\left[C_{\alpha} C_{1} \Gamma(1-\alpha) \delta / 2\right]^{1 / 1-\alpha}$.

Proof. From the variation of constants formula (1.17),

$$
x(t ; \xi, \eta, \varepsilon)=e^{-A t} \xi+\int_{0}^{t} e^{-A(t-s)} f(x(s ; \xi, \eta, \varepsilon), y(s ; \xi, \eta, \varepsilon), \varepsilon) d s
$$


Since $f(x(s ; \xi, \eta, \varepsilon), y(s ; \xi, \eta, \varepsilon), \varepsilon) \in X$ for $(x(s ; \xi, \eta, \varepsilon), y(s ; \xi, \eta, \varepsilon)) \in$ $B\left(\delta_{2}\right), 0 \leq s \leq T, \varepsilon \in\left[-\varepsilon_{2}, \varepsilon_{2}\right]$, by $(1.20)$,

$$
\begin{aligned}
& \left|e^{-A(t-s)} f(x(s ; \xi, \eta, \varepsilon), y(s ; \xi, \eta, \varepsilon), \varepsilon)\right|_{\alpha} \\
& \quad \leq \frac{C_{\alpha}}{(t-s)^{\alpha}}|f(x(s ; \xi, \eta, \varepsilon), y(s ; \xi, \eta, \varepsilon), \varepsilon)| .
\end{aligned}
$$

By (1.12) and (1.13), we have

$$
|f(x(s ; \xi, \eta, \varepsilon), y(s ; \xi, \eta, \varepsilon), \varepsilon)| \leq C_{1} \delta_{2}|x(s ; \xi, \eta, \varepsilon)|_{\alpha} .
$$

Thus,

$$
|x(t ; \xi, \eta, \varepsilon)|_{\alpha} \leq e^{-\mu t}|\xi|_{\alpha}+\int_{0}^{t} \frac{C_{\alpha}}{(t-s)^{\alpha}} e^{-\mu(t-s)} C_{1} \delta_{2}|x(s ; \xi, \eta, \varepsilon)|_{\alpha} d s
$$

Let $u(t)=e^{\mu t}|x(t ; \xi, \eta, \varepsilon)|_{\alpha}$. Then

$$
u(t) \leq u(0)+\int_{0}^{t} \frac{C_{\alpha} C_{1} \delta_{2}}{(t-s)^{\alpha}} u(s) d s
$$

By Lemma 1.1, the desired estimate is obtained.

Next, we will derive an estimate for the exponential growth of

$$
\left(D_{(\xi, \eta)} x(t ; \xi, \eta, \varepsilon), D_{(\xi, \eta)} y(t ; \xi, \eta, \varepsilon)\right)
$$

which is the unique solution of the variational equation (1.18) with initial value $I$, the identity map on $X^{\alpha} \times \mathbf{R}$.

By the remarks after Lemma 1.1, $\left[1+E_{\alpha}\left(t \theta\left(\delta_{2}\right)\right)\right] \cdot(1-\alpha) \exp \left(-t \theta\left(\delta_{2}\right)\right)$ is bounded on $(0,+\infty)$. Hence, there exist $0<\delta_{3}<\delta_{2}$, a constant $C_{2} \geq 2$ and a fixed $\gamma_{1}>0$ such that

$$
\begin{gathered}
\sigma=(\mu-\lambda) / 4-\theta(\delta) \geq \gamma_{1}>\theta(\delta)>0 \quad \text { for every } 0 \leq \delta \leq \delta_{3}, \\
\tilde{\mu}=(\mu-\lambda) / 4<\mu, \\
\tilde{\lambda}=(\mu+\lambda) / 2>\lambda, \\
\left(1+E_{\alpha}(t \theta(\delta))\right) e^{-\sigma t} \leq C_{2}, \quad 0 \leq t \leq+\infty, 0 \leq \delta \leq \delta_{3}, \\
\int_{0}^{+\infty} \frac{1}{s^{\alpha}} e^{(-\mu+\tilde{\mu}) s} d s \leq C_{2}, \\
\int_{0}^{+\infty} e^{(-\tilde{\lambda}+\lambda) s} d s \leq C_{2}, \\
\int_{0}^{+\infty} e^{(-\mu+\tilde{\mu}) s} d s \leq C_{2} .
\end{gathered}
$$

In the following, $\tilde{\tilde{\lambda}} \in(0, \lambda)$ is a given constant, and $\delta_{4}>0$ is a small constant satisfying

$$
\begin{aligned}
\delta_{4} \leq \min \left\{\delta_{3},(\lambda-\tilde{\lambda}) /\left(2 C_{1}\right), 1 /\left(2 \sqrt{C_{1}} C_{2}\right),(\lambda+1) /\left(8 C_{1}\right),\right. \\
\left.1 /\left[2 C_{\alpha} C_{1} C_{2}\left(3+2 C_{2}\right)+8 C_{1} C_{2}\right]\right\} .
\end{aligned}
$$


To simplify the notation, we will use $|\cdot|_{\alpha}$ and $|\cdot|$ as the operator norms in spaces $L\left(X^{\alpha} \times \mathbf{R}, X^{\alpha}\right)$ and $L\left(X^{\alpha} \times \mathbf{R}, \mathbf{R}\right)$, respectively. Also, we will use $\|\cdot\|_{\alpha}$ as a norm in $X^{\alpha} \times \mathbf{R}$ which is defined by

$$
\|(x, y)\|_{\alpha}=|x|_{\alpha}+|y|, \quad(x, y) \in X^{\alpha} \times \mathbf{R} .
$$

Lemma 1.3. Let $\tilde{\tilde{\lambda}}, \delta_{4}$ be as above, and $\tilde{\mu}, \tilde{\lambda}$ be as in (1.22) and (1.23). Let $T>0$. Suppose $(x(t ; \xi, \eta, \varepsilon), y(t ; \xi, \eta, \varepsilon)) \in B\left(\delta_{4}\right)$ for $0 \leq t \leq T$. Then

$$
\begin{array}{cc}
\left|D_{(\xi, \eta)} x(t ; \xi, \eta, \varepsilon)\right|_{\alpha} \leq 2 e^{-\mu t}, & 0 \leq t \leq T, \varepsilon \in\left[-\varepsilon_{2}, \varepsilon_{2}\right], \\
\left|D_{(\xi, \eta)} y(t ; \xi, \eta, \varepsilon)\right| \leq 2 e^{\tilde{\lambda} t}, & 0 \leq t \leq T, \varepsilon \in\left[-\varepsilon_{2}, \varepsilon_{2}\right], \\
\left|D_{\eta} y(t ; \xi, \eta, \varepsilon)\right| \geq \frac{1}{2} e^{\tilde{\tilde{\lambda} t}}, & 0 \leq t \leq T, \varepsilon \in\left[-\varepsilon_{2}, \varepsilon_{2}\right] .
\end{array}
$$

Proof. Note that $\left(D_{(\xi, \eta)} x(t ; \xi, \eta, \varepsilon), D_{(\xi, \eta)} y(t ; \xi, \eta, \varepsilon)\right)$ is the unique solution of $(1.18)$ such that $\left(D_{(\xi, \eta)} x(0 ; \xi, \eta, \varepsilon), D_{(\xi, \eta)} y(0 ; \xi, \eta, \varepsilon)\right)=I$, the identity on $X^{\alpha} \times \mathbf{R}$ (see Henry [8, pp. 64-65]).

Define a metric space

$$
\widetilde{V}=\left\{(p, q): p:[0, T] \rightarrow L\left(X^{\alpha} \times \mathbf{R}, X^{\alpha}\right) \text { and } q:[0, T] \rightarrow L\left(X^{\alpha} \times \mathbf{R}, \mathbf{R}\right)\right.
$$

are continuous in the operator norms $|\cdot|_{\alpha},|\cdot|$, respectively $\}$

with metric

$$
d\left(\left(p_{1} q_{1}\right),\left(p_{2}, q_{2}\right)\right)=\max _{0 \leq t \leq T}\left(\left|p_{1}(t)-p_{2}(t)\right|_{\alpha}+\left|q_{1}(t)-q_{2}(t)\right|\right),
$$

where $|\cdot|_{\alpha}$ and $|\cdot|$ also denote the norms in $L\left(X^{\alpha} \times \mathbf{R}, X^{\alpha}\right)$ and $L\left(X^{\alpha} \times \mathbf{R}, \mathbf{R}\right)$, respectively.

Let $V$ be the subset in $\tilde{V}$ defined by

$$
\begin{array}{r}
V=\left\{(p, q):(p, q) \in \widetilde{V},(p(0), q(0))=I, \text { the identity map on } X^{\alpha} \times \mathbf{R},\right. \\
\text { and } \left.|p(t)|_{\alpha} \leq 2 e^{-\tilde{\mu} t},|q(t)| \leq 2 e^{\tilde{\lambda} t}, 0 \leq t \leq T\right\} .
\end{array}
$$

Clearly, $V$ is a closed subset of $\tilde{V}$. Let $\Phi: V \rightarrow \widetilde{V},(\bar{p}, \bar{q})=\Phi(p, q)$, be defined by

$$
\begin{aligned}
& \bar{p}(t)=e^{-A t} p(0)+\int_{0}^{t} e^{-A(t-s)}\left[D_{x} f(x(s ; \xi, \eta, \varepsilon), y(s ; \xi, \eta, \varepsilon), \varepsilon) p(s)\right. \\
&\left.\quad D_{y} f(x(s ; \xi, \eta, \varepsilon), y(s ; \xi, \eta, \varepsilon), \varepsilon) q(s)\right] d s, \\
& \bar{q}(t)=e^{\lambda t} q(0)+\int_{0}^{t} e^{\lambda(t-s)}\left[D_{x} g(x(s ; \xi, \eta, \varepsilon), y(s ; \xi, \eta, \varepsilon, \varepsilon), \varepsilon) p(s)\right. \\
&\left.+D_{y} g(x(s ; \xi, \eta, \varepsilon), y(s ; \xi, \eta, \varepsilon), \varepsilon) q(s)\right] d s .
\end{aligned}
$$

First we show that $\Phi$ maps $V$ into itself. Because of (1.12), (1.14), (1.16), (1.19) and $(p(0), q(0))=I$, by using similar arguments as in the proof of 
Lemma 1.2, we have

$$
\begin{aligned}
|\bar{p}(t)|_{\alpha} \leq & e^{-\mu t}+\int_{0}^{t} \frac{C_{\alpha}}{(t-s)^{\alpha}} 3 C_{1} \delta_{4} e^{-\mu(t-s)}|p(s)|_{\alpha} d s \\
& +\int_{0}^{t} \frac{C_{\alpha}}{(t-s)^{\alpha}} 2 C_{1} e^{-\mu(t-s)}|x(s ; \xi, \eta, \varepsilon)|_{\alpha}|q(s)| d s \\
= & I_{1}+I_{2}+I_{3} \text { for }(p, q) \in V .
\end{aligned}
$$

Hence, we have $I_{1}=e^{-\mu t}$ and

$$
\begin{aligned}
I_{2} & =\int_{0}^{t} \frac{C_{\alpha}}{(t-s)^{\alpha}} 3 C_{1} \delta_{4} e^{-\mu(t-s)}|p(s)|_{\alpha} d s \\
& \leq 6 C_{\alpha} C_{1} \delta_{4} \int_{0}^{t} \frac{1}{(t-s)^{\alpha}} e^{-\mu(t-s)} e^{-\tilde{\mu} s} d s \\
& =6 C_{\alpha} C_{1} \delta_{4} e^{-\tilde{\mu} t} \int_{0}^{t} \frac{1}{(t-s)^{\alpha}} e^{(-\mu+\tilde{\mu})(t-s)} d s .
\end{aligned}
$$

By (1.25) we have

$$
I_{2} \leq 6 C_{\alpha} C_{1} C_{2} \delta_{4} e^{-\tilde{\mu} t}
$$

By Lemma 1.2 and (1.24), we have

$$
|x(t ; \xi, \eta, \varepsilon)|_{\alpha} \leq|\xi|_{\alpha}\left(1+E_{\alpha}\left(t \theta\left(\delta_{4}\right)\right)\right) e^{-\mu t} \leq C_{2} \delta_{4} e^{(-\mu+\tilde{\mu}) t} .
$$

Hence,

By (1.25) we have

$$
\begin{aligned}
I_{3} & =\int_{0}^{t} \frac{C_{\alpha}}{(t-s)^{\alpha}} 2 C_{1} e^{-\mu(t-s)}|x(s ; \xi, \eta, \varepsilon)|_{\alpha}|q(s)| d s \\
& \leq 4 C_{\alpha} C_{1} C_{2} \delta_{4} \int_{0}^{t} \frac{1}{(t-s)^{\alpha}} e^{-\mu(t-s)} e^{(-\mu+\tilde{\mu}) s} e^{\tilde{\lambda} s} d s \\
& \leq 4 C_{\alpha} C_{1} C_{2} \delta_{4} e^{-\tilde{\mu} t} \int_{0}^{t} \frac{1}{(t-s)^{\alpha}} e^{(-\mu+\tilde{\mu})(t-s)} d s .
\end{aligned}
$$

Therefore,

$$
I_{3} \leq 4 C_{\alpha} C_{1} C_{2}^{2} \delta_{4} e^{-\tilde{\mu} t} .
$$

$$
|\bar{p}(t)|_{\alpha} \leq\left(1+C_{\alpha} C_{1} C_{2}\left(6+4 C_{2}\right) \delta_{4}\right) e^{-\tilde{\mu} t} \leq 2 e^{-\tilde{\mu} t}
$$

since $\delta_{4} \leq 1 /\left[C_{\alpha} C_{1} C_{2}\left(6+4 C_{2}\right)\right]$ by (1.28). Similarly,

$$
\begin{aligned}
|\bar{q}(t)| & \leq e^{\lambda t}+\int_{0}^{t} e^{\lambda(t-s)} 2 C_{1} \delta_{4}|p(s)|_{\alpha}+\int_{0}^{t} e^{\lambda(t-s)} 2 C_{1} \delta_{4}|q(s)| d s \\
& =\tilde{I}_{1}+\tilde{I}_{2}+\tilde{I}_{3},
\end{aligned}
$$

where $\tilde{I}_{1}=e^{\lambda t}$ and

$$
\begin{aligned}
\tilde{I}_{2} & =\int_{0}^{t} e^{\lambda(t-s)} 2 C_{1} \delta_{4}|p(s)|_{\alpha} d s \\
& \leq 4 C_{1} \delta_{4} \int_{0}^{t} e^{\lambda(t-s)} e^{-\tilde{\mu} s} d s \\
& =4 C_{1} \delta_{4} e^{\tilde{\lambda} t} \int_{0}^{t} e^{(-\tilde{\lambda}+\lambda)(t-s)} e^{-(\tilde{\lambda}+\tilde{\mu}) s} d s .
\end{aligned}
$$


Because of $(1.23),(1.27)$ and $-(\tilde{\lambda}+\tilde{\mu})=-\mu+\tilde{\mu}$,

$$
\begin{gathered}
\tilde{I}_{2} \leq 4 C_{1} C_{2} \delta_{4} e^{\lambda t}, \\
\tilde{I}_{3}=\int_{0}^{t} e^{\lambda(t-s)} 2 C_{1} \delta_{4}|q(s)| d s \\
\leq 4 C_{1} \delta_{4} \int_{0}^{t} e^{\lambda(t-s)} e^{\dot{\lambda} s} d s \\
=4 C_{1} \delta_{4} e^{\tilde{\lambda} t} \int_{0}^{t} e^{(-\tilde{\lambda}+\lambda)(t-s)} d s .
\end{gathered}
$$

By (1.26) we have $\tilde{I}_{3} \leq 4 C_{1} C_{2} \delta_{4} e^{\tilde{\lambda} t}$. Hence,

$$
|\bar{q}(t)| \leq\left(1+8 C_{1} C_{2} \delta_{4}\right) e^{\bar{\lambda} t} \leq 2 e^{\bar{\lambda} t}
$$

since $\delta \leq 1 /\left[8 C_{1} C_{2}\right]$ by (1.28). It follows that $\Phi: V \rightarrow V$. Next, we will equip $V$ with another metric $\bar{d}$ such that $(V, \bar{d})$ is complete and the map $\Phi: V \rightarrow V$ under this new topology $(V, \bar{d})$ is a contraction mapping.

Let $\left(p_{1}, q_{1}\right),\left(p_{2}, q_{2}\right) \in V$ and define

$$
\bar{d}\left(\left(p_{1}, q_{1}\right),\left(p_{2}, q_{2}\right)\right)=\max _{0 \leq t \leq T}\left(e^{\tilde{\mu} t}\left|p_{1}(t)-p_{2}(t)\right|_{\alpha}+e^{-\tilde{\lambda} t}\left|q_{1}(t)-q_{2}(t)\right|\right) .
$$

It is easy to verify that $(V, \bar{d})$ is complete. To show $\Phi:(V, \bar{d}) \rightarrow(V, \bar{d})$ is contractive, let $\left(\bar{p}_{i}, \bar{q}_{i}\right)=\Phi\left(p_{i}, q_{i}\right),\left(p_{i}, q_{i}\right) \in V$ for $i=1,2$. From (1.29), we have

$$
\begin{aligned}
\left|\bar{p}_{1}(t)-\bar{p}_{2}(t)\right|_{\alpha} \leq & \int_{0}^{t} \frac{C_{\alpha}}{(t-s)^{\alpha}} 3 C_{1} \delta_{4} e^{-\mu(t-s)}\left|p_{1}(s)-p_{2}(s)\right|_{\alpha} d s \\
& +\int_{0}^{t} \frac{C_{\alpha}}{(t-s)^{\alpha}} 2 C_{1} e^{-\mu(t-s)}|x(s ; \xi, \eta, \varepsilon)|_{\alpha}\left|q_{1}(s)-q_{1}(s)\right| d s \\
= & \tilde{I}_{1}+\tilde{\tilde{I}}_{2},
\end{aligned}
$$

where

$$
\begin{aligned}
\tilde{\tilde{I}}_{1} & =3 C_{\alpha} C_{1} \delta_{4} \int_{0}^{t} \frac{1}{(t-s)^{\alpha}} e^{-\mu(t-s)}\left|p_{1}(s)-p_{2}(s)\right|_{\alpha} d s \\
& =3 C_{\alpha} C_{1} \delta_{4} \int_{0}^{t} \frac{1}{(t-s)^{\alpha}} e^{-\mu(t-s)} e^{-\tilde{\mu} s}\left(e^{\tilde{\mu} s}\left|p_{1}(s)-p_{2}(s)\right|_{\alpha}\right) d s \\
& \leq 3 C_{\alpha} C_{1} \delta_{4} e^{-\tilde{\mu} t} \int_{0}^{t} \frac{1}{(t-s)^{\alpha}} e^{(-\mu+\tilde{\mu})(t-s)} d s \bar{d}\left(\left(p_{1}, q_{1}\right),\left(p_{2}, q_{2}\right)\right) .
\end{aligned}
$$

By (1.25) we have

$$
\tilde{\tilde{I}}_{1} \leq 3 C_{\alpha} C_{1} C_{2} \delta_{4} e^{-\tilde{\mu} t} \bar{d}\left(\left(p_{1}, q_{1}\right),\left(p_{2}, q_{2}\right)\right)
$$


Because of (1.27) and $-\mu+2 \tilde{\mu}+\tilde{\lambda}=0$ we have

$$
\begin{aligned}
\tilde{\tilde{I}}_{2} & =\int_{0}^{t} \frac{C_{\alpha}}{(t-s)^{\alpha}} 2 C_{1} e^{-\mu(t-s)}|x(s ; \xi, \eta, \varepsilon)|_{\alpha}\left|q_{1}(s)-q_{2}(s)\right| d s \\
& \leq 2 C_{\alpha} C_{1} C_{2} \delta_{4} \int_{0}^{t} \frac{1}{(t-s)^{\alpha}} e^{-\mu(t-s)} e^{(-\mu+\tilde{\mu}+\tilde{\lambda}) s}\left(e^{-\tilde{\lambda} s}\left|q_{1}(s)-q_{2}(s)\right|\right) d s \\
& \leq 2 C_{\alpha} C_{1} C_{2} \delta_{4} e^{-\tilde{\mu} t} \int_{0}^{t} \frac{1}{(t-s)^{\alpha}} e^{(-\mu+\tilde{\mu})(t-s)} e^{(-\mu+2 \tilde{\mu}+\tilde{\lambda}) s} d s \bar{d}\left(\left(p_{1}, q_{1}\right),\left(p_{2}, q_{2}\right)\right) \\
& =2 C_{\alpha} C_{1} C_{2} \delta_{4} e^{-\tilde{\mu} t} \int_{0}^{t} \frac{1}{(t-s)^{\alpha}} e^{(-\mu+\tilde{\mu})(t-s)} d s \bar{d}\left(\left(p_{1}, q_{1}\right),\left(p_{2}, q_{2}\right)\right) .
\end{aligned}
$$

By (1.25) we have

$$
\tilde{\tilde{I}}_{2} \leq 2 C_{\alpha} C_{1} C_{2}^{2} \delta_{4} e^{-\tilde{\mu} t} \bar{d}\left(\left(p_{1}, q_{1}\right),\left(p_{2}, q_{2}\right)\right)
$$

Hence,

$$
e^{\tilde{\mu} t}\left|\bar{p}_{1}(t)-\bar{p}_{2}(t)\right|_{\alpha} \leq C_{\alpha} C_{1} C_{2}\left(3+2 C_{2}\right) \delta_{4} \bar{d}\left(\left(p_{1}, q_{1}\right),\left(p_{1}, q_{2}\right)\right)
$$

Moreover, from (1.26) we have

$$
\begin{aligned}
\left|\bar{q}_{1}(t)-\bar{q}_{2}(t)\right| \leq & \int_{0}^{t} e^{\lambda(t-s)} 2 C_{1} \delta_{4}\left|p_{1}(s)-p_{2}(s)\right|_{\alpha} d s \\
& +\int_{0}^{t} e^{\lambda(t-s)} 2 C_{1} \delta_{4}\left|q_{1}(s)-q_{2}(s)\right| d s \\
= & \tilde{\tilde{I}}_{1}+\tilde{\tilde{I}}_{2},
\end{aligned}
$$

where

$$
\begin{aligned}
\tilde{\tilde{I}}_{1} & =\int_{0}^{t} e^{\lambda(t-s)} 2 C_{1} \delta_{4}\left|p_{1}(s)-p_{2}(s)\right| d s \\
& =2 C_{1} \delta_{4} \int_{0}^{t} e^{\lambda(t-s)} e^{-\tilde{\mu} s}\left(e^{\tilde{\mu} s}\left|p_{1}(s)-p_{2}(s)\right|\right) d s \\
& =2 C_{1} \delta_{4} e^{\tilde{\lambda} t} \int_{0}^{t} e^{(-\tilde{\lambda}+\lambda)(t-s)} e^{-(\tilde{\mu}+\tilde{\lambda}) s} d s \bar{d}\left(\left(p_{1}, q_{1}\right),\left(p_{2}, q_{2}\right)\right)
\end{aligned}
$$

By (1.26) we have

$$
\begin{aligned}
& \tilde{\tilde{I}}_{1} \leq 2 C_{1} C_{2} \delta_{4} e^{\tilde{\lambda} t} \bar{d}\left(\left(p_{1}, q_{1}\right),\left(p_{2}, q_{2}\right)\right) \\
\tilde{\tilde{I}}_{2}= & \int_{0}^{t} e^{\lambda(t-s)} 2 C_{1} \delta_{4}\left|q_{1}(s)-q_{2}(s)\right| d s \\
= & 2 C_{1} \delta_{4} \int_{0}^{t} e^{\lambda(t-s)} e^{\tilde{\lambda} s}\left(e^{-\tilde{\lambda} s}\left|q_{1}(s)-q_{2}(s)\right|\right) d s \\
\leq & 2 C_{1} \delta_{4} e^{\bar{\lambda} t} \int_{0}^{t} e^{(-\tilde{\lambda}+\lambda)(t-s)} d s \bar{d}\left(\left(p_{1}, q_{1}\right),\left(p_{2}, q_{2}\right)\right) .
\end{aligned}
$$

By (1.26) we have

$$
\tilde{\tilde{I}}_{2} \leq 2 C_{1} C_{2} \delta_{4} e^{\tilde{\lambda} t} \bar{d}\left(\left(p_{1}, q_{1}\right),\left(p_{2}, q_{2}\right)\right)
$$


Hence,

$$
e^{-\bar{\lambda} t}\left|\bar{q}_{1}(t)-\bar{q}_{2}(t)\right| \leq 4 C_{1} C_{2} \delta_{4} \bar{d}\left(\left(p_{1}, q_{1}\right),\left(p_{2}, q_{2}\right)\right) .
$$

Therefore, from (1.32) and the estimate above we have

$$
\begin{aligned}
\bar{d}\left(\left(\bar{p}_{1}, \bar{q}_{1}\right),\left(\bar{p}_{2}, \bar{q}_{2}\right)\right) & =\max _{0 \leq t \leq T}\left(e^{\tilde{\mu} t}\left|\bar{p}_{1}(t)-\bar{p}_{2}(t)\right|_{\alpha}+e^{-\bar{\lambda} t}\left|\bar{q}_{1}(t)-\bar{q}_{2}(t)\right|\right) \\
& \leq\left[C_{\alpha} C_{1} C_{2}\left(3+2 C_{2}\right)+4 C_{1} C_{2}\right] \delta_{4} \bar{d}\left(\left(p_{1}, q_{1}\right),\left(p_{2}, q_{2}\right)\right) \\
& \leq \frac{1}{2} \bar{d}\left(\left(p_{1}, q_{1}\right),\left(p_{2}, q_{2}\right)\right)
\end{aligned}
$$

since $\delta_{4} \leq 1 /\left[C_{\alpha} C_{1} C_{2}\left(6+4 C_{2}\right)+8 C_{1} C_{2}\right]$ by (1.28). By the contraction mapping theorem, there exists a unique $(\tilde{p}, \tilde{q}) \in V$, such that $(\tilde{p}, \tilde{q})=\Phi(\tilde{p}, \tilde{q})$. By the uniqueness of the solution of (1.18), we have

$$
\tilde{p}(t)=D_{(\xi, \eta)} x(t ; \xi, \eta, \varepsilon), \quad \tilde{q}(t)=D_{(\xi, \eta)} y(t ; \xi, \eta, \varepsilon) .
$$

Thus, the first two estimates of the theorem hold:

$$
\begin{array}{cc}
\left|D_{(\xi, \eta)} x(t ; \xi, \eta, \varepsilon)\right|_{\alpha} \leq 2 e^{-\tilde{\mu} t}, & 0 \leq t \leq T, \varepsilon \in\left[-\varepsilon_{2}, \varepsilon_{2}\right], \\
\left|D_{(\xi, \eta)} y(t ; \xi, \eta, \varepsilon)\right| \leq 2 e^{\tilde{\lambda} t}, & 0 \leq t \leq T, \varepsilon \in\left[-\varepsilon_{2}, \varepsilon_{2}\right] .
\end{array}
$$

Next, we show

$$
\left|D_{\eta} y(t ; \xi, \eta, \varepsilon)\right| \geq \frac{1}{2} e^{\tilde{\tilde{\lambda}}}, \quad 0 \leq t \leq T .
$$

By (1.18), we have

$D_{\eta} y(t ; \xi, \eta, \varepsilon)$

$$
\begin{aligned}
& =e^{\lambda t}+\int_{0}^{t} e^{\lambda(t-s)}\left[D_{x} g(x(s ; \xi, \eta, \varepsilon), y(s ; \xi, \eta, \varepsilon), \varepsilon)\right. \\
& \cdot D_{\eta} x(s ; \xi, \eta, \varepsilon)+D_{y} g(x(s ; \xi, \eta, \varepsilon), y(s ; \xi, \eta, \varepsilon), \varepsilon) \\
& \left.\cdot D_{\eta} y(s ; \xi, \eta, \varepsilon)\right] d s,
\end{aligned}
$$

Let

$$
T_{1}=\sup \left\{t: 0 \leq t \leq T, D_{\eta} y(t ; \xi, \eta, \varepsilon) \geq 0\right\} .
$$

Since $D_{\eta} y(0, \xi, \eta, \varepsilon)=1, T_{1}>0$. We will show $T_{1}=T$. Suppose the contrary, $T_{1}<T, D_{\eta} y\left(T_{1}, \xi, \eta, \varepsilon\right)=0$. By differentiating (1.33) in $t$, we have

$$
\begin{aligned}
\frac{d D_{\eta} y(t ; \xi, \eta, \varepsilon)}{d t}= & {\left[\lambda+D_{y} g(x(t ; \xi, \eta, \varepsilon), y(t ; \xi, \eta, \varepsilon), \varepsilon)\right] \cdot D_{\eta} y(t ; \xi, \eta, \varepsilon) } \\
& +D_{x} g(x(t ; \xi, \eta, \varepsilon), y(t ; \xi, \eta, \varepsilon), \varepsilon) \cdot D_{\eta} x(t ; \xi, \eta, \varepsilon)
\end{aligned}
$$


Thus, by (1.15), (1.16), (1.28) and (1.30), we have for $0 \leq t \leq T_{1}$

$$
\begin{aligned}
\frac{d D_{\eta} y(t ; \xi, \eta, \varepsilon)}{d t} & \geq\left[\lambda-2 C_{1} \delta_{4}\right] D_{\eta} y(t ; \xi, \eta, \varepsilon)-2 C_{1} C_{2} \delta_{4}^{2} e^{(-\mu+\tilde{\mu}) t} \\
& \geq \widetilde{\tilde{\lambda}} D_{\eta} y(t ; \xi, \eta, \varepsilon)-2 C_{1} C_{2} \delta_{4}^{2} e^{(-\mu+\tilde{\mu}) t} .
\end{aligned}
$$

It follows from the above inequality and (1.28) that

$$
D_{\eta} y(t ; \xi, \eta, \varepsilon) \geq \frac{1}{2} e^{\tilde{\lambda} t}, \quad 0 \leq t \leq T_{1} .
$$

Thus, $D_{\eta} y\left(T_{1} ; \xi, \eta, \varepsilon\right) \geq \frac{1}{2} e^{\tilde{\lambda} T_{1}} \neq 0$. This is a contradiction. Hence, $T_{1}=T$ and the desired estimate holds.

By (1.21) and (1.24), we have

$$
\left|\left[1+E_{\alpha}(t \theta(\delta))\right] e^{-\mu t}\right| \leq C_{2} \text { for } t \geq 0,0<\delta \leq \delta_{4} .
$$

Let

$$
\Omega\left(\delta_{4}, \rho, \varepsilon\right)=\left\{(x, y):|x|_{\alpha} \leq \delta_{4} / 2 C_{2},\left|y-h^{s}(x, \varepsilon)\right|<\rho\right\},
$$

$$
\Omega^{+}\left(\delta_{4}, \rho, \varepsilon\right)=\left\{(x, y):(x, y) \in \Omega\left(\delta_{4}, \rho, \varepsilon\right), 0<y-h^{s}(x, \varepsilon)<\rho\right\}
$$

$$
\Omega^{-}\left(\delta_{4}, \rho, \varepsilon\right)=\left\{(x, y):(x, y) \in \Omega\left(\delta_{4}, \rho, \varepsilon\right),-\rho<y-h^{s}(x, \varepsilon)<0\right\},
$$

where $\varepsilon \in\left[-\varepsilon_{2}, \varepsilon_{2}\right]$. Note that by (1.11) and (1.28), $\delta_{4} / 4-C_{1} \delta_{4}^{2}>0$ and $\left|h^{s}(x, \varepsilon)\right| \leq C_{1} \delta_{4}^{2}$ for $|x|_{\alpha} \leq \delta_{4}$ and $\varepsilon \in\left[-\varepsilon_{2}, \varepsilon_{2}\right]$. Thus, for every $(x, y) \in$ $\Omega\left(\delta_{4}, \rho, \varepsilon\right)$ with $0<\rho<\delta_{4} / 4-C_{1} \delta_{4}^{2},|y| \leq\left|y-h^{s}(x, \varepsilon)\right|+\left|h^{s}(x, \varepsilon)\right|<\delta_{4} / 4$. Hence, $\Omega\left(\delta_{4}, \rho, \varepsilon\right) \subset B\left(\delta_{4} / 2\right)$ for every $\varepsilon \in\left[-\varepsilon_{2}, \varepsilon_{2}\right]$.

Lemma 1.4. Let $(x(t ; \xi, \eta, \varepsilon), y(t ; \xi, \eta, \varepsilon))$ be a solution of $(1.1)$ in $B\left(\delta_{4}\right)$ and $0<\rho<\delta_{4} / 4-C_{1} \delta_{4}^{2}$. Then for every $(\xi, \eta) \in \Omega^{+}\left(\delta_{4}, \rho, \varepsilon\right) \cup \Omega^{-}\left(\delta_{4}, \rho, \varepsilon\right)$, there exists $\tau(\xi, \eta, \varepsilon)>0$ such that

$$
\begin{gathered}
y(\tau(\xi, \eta, \varepsilon) ; \xi, \eta, \varepsilon)-y\left(\tau(\xi, \eta, \varepsilon) ; \xi, h^{s}(\xi, \varepsilon), \varepsilon\right) \\
= \begin{cases}\delta_{4} / 2, & \text { if }(\xi, \eta) \in \Omega^{+}\left(\delta_{4}, \rho, \varepsilon\right), \\
-\delta_{4} / 2, & \text { if }(\xi, \eta) \in \Omega^{-}\left(\delta_{4}, \rho, \varepsilon\right) .\end{cases}
\end{gathered}
$$

Furthermore, $\tau: \Omega^{+}\left(\delta_{4}, \rho, \varepsilon\right) \cup \Omega^{-}\left(\delta_{4}, \rho, \varepsilon\right) \rightarrow \mathbf{R}$ is $C^{2}$ and satisfies

$$
\begin{gathered}
\tau(\xi, \eta, \varepsilon) \geq \frac{1}{\tilde{\lambda}} \ln \frac{\delta_{4}}{4\left|\eta-h^{s}(\xi, \varepsilon)\right|}, \quad \tau(\xi, \eta, \varepsilon) \leq \frac{1}{\approx} \ln \frac{\delta_{4}}{\left|\eta-h^{s}(\xi, \varepsilon)\right|}, \\
\left|D_{(\xi, \eta)} \tau(\xi, \eta, \varepsilon)\right| \leq \frac{8}{\lambda \delta_{4}-8 C_{1} \delta_{4}^{2}} e^{\lambda_{\tau} \tau(\xi, \eta, \varepsilon)}
\end{gathered}
$$

Proof. If $(\xi, \eta)$ is in

$$
\Omega^{+}\left(\delta_{4}, \rho, \varepsilon\right) \cup \Omega^{-}\left(\delta_{4}, \rho, \varepsilon\right) \subset \Omega\left(\delta_{4}, \rho, \varepsilon\right)-W_{\mathrm{loc}}^{s}(\varepsilon),
$$


then $(x(t ; \xi, \eta, \varepsilon), y(t ; \xi, \eta, \varepsilon))$ is not in $W_{\text {loc }}^{s}(\varepsilon)$. Hence, the solution cannot stay in $B\left(\delta_{4}\right)$ for all $t>0$. If $|\xi| \leq \delta_{4} / 2 C_{1}$, then by Lemma 1.2

$$
|x(t ; \xi, \eta, \varepsilon)|_{\alpha}<C_{2} \delta_{4} / 2 C_{2}=\delta_{4} / 2 \text { for } 0 \leq t \leq T,
$$

where $T>0$ is such that the solution $(x(t ; \xi, \eta, \varepsilon), y(t ; \xi, \eta, \varepsilon))$ is in $B\left(\delta_{4}\right)$ for all $0 \leq t \leq T$. Hence, it has to leave $B\left(\delta_{4}\right)$ through either hyperplane $y=\delta_{4}$ or hyperplane $y=-\delta_{4}$. Therefore,

$$
\tilde{\tau}=\inf \left\{t>0:|y(t ; \xi, \eta, \varepsilon)|=\delta_{4}\right\}
$$

is well defined.

Let

$$
\Delta(t ; \xi, \eta, \varepsilon)=y(t ; \xi, \eta, \varepsilon)-y\left(t ; \xi, h^{s}(\xi, \varepsilon), \varepsilon\right), \quad 0 \leq t \leq \tilde{\tau} .
$$

Note that $\Delta$ is $C^{2}$. Since $(\xi, \eta) \in \Omega\left(\delta_{4}, \rho, \varepsilon\right)$,

$$
|\Delta(0, \xi, \eta, \varepsilon)|=\left|\eta-h^{s}(\xi, \varepsilon)\right|<\rho<\delta_{4} / 4-C_{1} \delta_{4}^{2}<\delta_{4} / 2
$$

Since $\left(x\left(t ; \xi, h^{s}(\xi, \varepsilon), \varepsilon\right), y\left(t ; \xi, h^{s}(\xi, \varepsilon), \varepsilon\right)\right)$ is in $W_{\text {loc }}^{s}(\varepsilon)$, by (1.11) we have

$$
|\Delta(\tilde{\tau} ; \xi, \eta, \varepsilon)| \geq|y(\tilde{\tau} ; \xi, \eta, \varepsilon)|-\left|y\left(\tilde{\tau} ; \xi, h^{s}(\xi, \varepsilon), \varepsilon\right)\right| \geq \delta_{4}-C_{1} \delta_{4}^{2}>\delta_{4} / 2 .
$$

Hence, by the intermediate value theorem,

$$
\tau(\xi, \eta, \varepsilon)=\inf \left\{t: 0<t<\tilde{\tau},|\Delta(t ; \xi, \eta, \varepsilon)|=\delta_{4} / 2\right\}
$$

is well defined for every $(\xi, \eta) \in \Omega^{+}\left(\delta_{4}, \rho, \varepsilon\right) \cup \Omega^{-}\left(\delta_{4}, \rho, \varepsilon\right)$.

Next, we will prove that

$$
\Delta(\tau(\xi, \eta, \varepsilon) ; \xi, \eta, \varepsilon)= \begin{cases}\delta_{4} / 2, & \text { if }(\xi, \eta) \in \Omega^{+}\left(\delta_{4}, \rho, \varepsilon\right), \\ -\delta_{4} / 2, & \text { if }(\xi, \eta) \in \Omega^{-}\left(\delta_{4}, \rho, \varepsilon\right) .\end{cases}
$$

We only prove $\Delta(\tau(\xi, \eta, \varepsilon) ; \xi, \eta, \varepsilon)=\delta_{2} / 2$ for $(\xi, \eta) \in \Omega^{+}\left(\delta_{4}, \rho, \varepsilon\right)$ since the other case follows the same argument.

Suppose the contrary,

$$
\Delta(\tau(\xi, \eta, \varepsilon) ; \xi, \eta, \varepsilon)=-\delta_{4} / 2 .
$$

Since $\left(x\left(t ; \xi, h^{s}(\xi, \varepsilon), \varepsilon\right), y\left(t ; \xi, h^{s}(\xi, \varepsilon), \varepsilon\right)\right)$ is in $W_{\text {loc }}^{s}(\varepsilon)$, thus, by $(1.11)$ and (1.28) we have

$$
y(\tau(\xi, \eta, \varepsilon) ; \xi, \eta, \varepsilon)=-\delta_{4} / 2+y\left(\tau(\xi, \eta, \varepsilon) ; \xi, h^{s}(\xi, \varepsilon), \varepsilon\right)<-\delta_{4} / 4
$$

Define

$$
d(t ; \xi, \eta, \varepsilon)=y(t ; \xi, \eta, \varepsilon)-h^{s}(x(t ; \xi, \eta, \varepsilon), \varepsilon) .
$$

Since $(\xi, \eta) \in \Omega^{+}\left(\delta_{4}, \rho, \varepsilon\right)$, we have

$$
d(0 ; \xi, \eta, \varepsilon)=\eta-h^{s}(\xi, \varepsilon)=\Delta(0 ; \xi, \eta, \varepsilon)>0 .
$$

But, by (1.40), (1.11) and (1.28) we have

$$
d(\tau(\xi, \eta, \varepsilon) ; \xi, \eta, \varepsilon)<-\delta_{4} / 4-h^{s}(x(\tau(\xi, \eta, \varepsilon), \varepsilon))<0
$$


Hence, by the intermediate value theorem there exists $\tau_{0}, 0<\tau_{0}<\tau(\xi, \eta, \varepsilon)$, such that

$$
d\left(\tau_{0} ; \xi, \eta, \varepsilon\right)=0 .
$$

This means $\left(x\left(\tau_{0} ; \xi, \eta, \varepsilon\right), y\left(\tau_{0} ; \xi, \eta, \varepsilon\right)\right) \in W_{\text {loc }}^{s}(\varepsilon)$ and, therefore,

$$
(x(t ; \xi, \eta, \varepsilon), y(t ; \xi, \eta, \varepsilon)) \subset W_{\mathrm{loc}}^{s}(\varepsilon) .
$$

This contradicts $(\xi, \eta) \in \Omega\left(\delta_{4}, \rho, \varepsilon\right)-W_{\text {loc }}^{s}(\varepsilon)$. So (1.39) holds.

Since $\Delta(t ; \xi, \eta, \varepsilon)$ is $C^{2}$ and

$$
\begin{aligned}
\frac{\partial \Delta}{\partial t}(t ; \xi, \eta, \varepsilon)= & \lambda \Delta(t ; \xi, \eta, \varepsilon)+g(x(t ; \xi, \eta, \varepsilon), y(t ; \xi, \eta, \varepsilon), \varepsilon) \\
& -g\left(x\left(t ; \xi, h^{s}(\xi, \varepsilon), \varepsilon\right), y\left(t ; \xi, h^{s}(\xi, \varepsilon), \varepsilon\right), \varepsilon\right),
\end{aligned}
$$

by $(1.15)$ and (1.28) we have

$$
\left.\frac{\partial \Delta}{\partial t}(t ; \xi, \eta, \varepsilon)\right|_{t=\tau(\xi, \eta, \varepsilon)} \geq \frac{\lambda \delta_{4}}{2}-4 C_{1} \delta_{4}^{2}>0
$$

for every $(\xi, \eta) \in \Omega^{+}\left(\delta_{4}, \rho, \varepsilon\right)$. By applying the implicit function theorem to the equation

$$
\Delta(\tau ; \xi, \eta, \varepsilon) \equiv \delta_{4} / 2, \quad(\xi, \eta) \in \Omega^{+}\left(\delta_{4}, \rho, \varepsilon\right), \varepsilon \in\left[-\varepsilon_{2}, \varepsilon_{2}\right],
$$

we have that $\tau$ is $C^{2}$. Moreover, by Lemma 1.3 we have

$$
\begin{aligned}
\delta_{4} / 2 & =\left|y(\tau(\xi, \eta, \varepsilon) ; \xi, \eta, \varepsilon)-y\left(\tau(\xi, \eta, \varepsilon) ; \xi, h^{s}(\xi, \varepsilon), \varepsilon\right)\right| \\
& \leq 2 e^{\dot{\lambda} \tau(\xi, \eta, \varepsilon)}\left|\eta-h^{s}(\xi, \eta)\right| \leq 2 e^{\dot{\lambda} \tau(\xi, \eta, \varepsilon)} \rho .
\end{aligned}
$$

Thus

$$
\tau(\xi, \eta, \varepsilon) \geq \frac{1}{\tilde{\lambda}} \ln \frac{\delta_{4}}{4\left|\eta-h^{s}(\xi, \varepsilon)\right|} .
$$

Similarly, by using Lemma 1.3 we can show

$$
\tau(\xi, \eta, \varepsilon) \leq \frac{1}{\approx} \ln \frac{\delta_{4}}{\left|\eta-h^{s}(\xi, \varepsilon)\right|}
$$

Finally, let $(\xi, \eta) \in \Omega^{+}(\delta, \rho, \varepsilon) \cup \Omega^{-}(\delta, \rho, \varepsilon)$. Using the chain rule and differentiating (1.39) with respect to $(\xi, \eta)$, we obtain

$$
0=\left.\frac{\partial \Delta}{\partial t}(t ; \xi, \eta, \varepsilon)\right|_{t=\tau(\xi, \eta, \varepsilon)} D_{(\xi, \eta)} \tau(\xi, \eta, \varepsilon)+\left.D_{(\xi, \eta)} \Delta(t ; \xi, \eta, \varepsilon)\right|_{t=\tau(\xi, \eta, \varepsilon)} .
$$

Thus, by (1.41) and Lemma 1.3 we obtain

$$
\left|D_{(\xi, \eta)} \tau(\xi, \eta, \varepsilon)\right| \leq \frac{8}{\lambda \delta_{4}-8 C_{1} \delta_{4}^{2}} e^{\tilde{\lambda} \tau(\xi, \eta, \varepsilon)} .
$$

The proof is complete.

The following may be found in Henry [8, p. 71]. 
Lemma 1.5. Let $(\xi, \eta) \in \Omega\left(\delta_{4}, \rho, \varepsilon\right), \varepsilon \in\left[-\varepsilon_{2}, \varepsilon_{2}\right], T>0$. For equation (1.1) there exists a constant $C_{3}$ independent of $\xi, \eta, \varepsilon_{2}, T$ and $\delta_{4}$ (see (1.28)) such that if the solution $(x(t ; \xi, \eta, \varepsilon), y(t ; \xi, \eta, \varepsilon))$ is in $B\left(\delta_{4}\right)$ for $0 \leq t \leq T$, then for every $0 \leq \beta<1, \frac{d}{d t} x(t ; \xi, \eta, \varepsilon) \in L\left(\mathbf{R}, X^{\beta}\right)$ and

$$
\left|\frac{d}{d t} x(t ; \xi, \eta, \varepsilon)\right|_{\beta} \leq C_{3} t^{\alpha-\beta-1} e^{-\mu t}, \quad 0<t \leq T, \varepsilon \in\left[-\varepsilon_{2}, \varepsilon_{2}\right] .
$$

Remark. In particular, choose $\beta=\alpha$. Then

$$
\left|\frac{d}{d t} x(t ; \xi, \eta, \varepsilon)\right|_{\alpha} \leq \frac{C_{3}}{t} e^{-\mu t} .
$$

Note that $x(\cdot ; \xi, \eta, \varepsilon) \in C^{1}[(0, T), X]$ but $x(\cdot ; \xi, \eta, \varepsilon) \notin C^{1}\left[(0, T), X^{\alpha}\right]$ in general if $\alpha \neq 0$. However, $\frac{d}{d t} x(t ; \xi, \eta, \varepsilon)$ is an element in $L\left(\mathbf{R}, X^{\alpha}\right)$ and bounded by $C_{3} e^{-\mu t} / t$ for $0<t \leq T$.

Lemma 1.6. Let

$$
x_{1}(\xi, \eta, \varepsilon)=x(\tau(\xi, \eta, \varepsilon) ; \xi, \eta, \varepsilon), \quad y_{1}(\xi, \eta, \varepsilon)=y(\tau(\xi, \eta, \varepsilon) ; \xi, \eta, \varepsilon),
$$

where $(\xi, \eta) \in \Omega^{+}\left(\delta_{4}, \rho, \varepsilon\right), \rho<\delta_{4} / 4-C_{1} \delta_{4}^{2}$ and $\varepsilon \in\left[-\varepsilon_{3}, \varepsilon_{3}\right]$. Then, there exists a constant $C_{4}\left(\delta_{4}\right)$ independent of $\xi, \eta, \varepsilon$ and $\varepsilon_{3}$, such that for $\left(\xi_{i}, \eta_{i}\right) \in$ $\Omega^{+}\left(\delta_{4}, \rho, \varepsilon\right), i=1,2$,

$$
\begin{gathered}
\left|x_{1}\left(\xi_{1}, \eta_{1}, \varepsilon\right)-x_{1}\left(\xi_{2}, \eta_{2}, \varepsilon\right)\right|_{\alpha}+\left|y_{1}\left(\xi_{1}, \eta_{1}, \varepsilon\right)-y_{1}\left(\xi_{2}, \eta_{2}, \varepsilon\right)\right| \\
\leq C_{4}\left(\delta_{4}\right) l\left(\xi_{1}, \eta_{1}, \xi_{2}, \eta_{2}\right)^{a}\left(\left|\xi_{1}-\xi_{2}\right|_{\alpha}+\left|\eta_{1}-\eta_{2}\right|\right),
\end{gathered}
$$

where $a=\min \{\tilde{\mu} / \tilde{\lambda},(\mu-\tilde{\lambda}) / \tilde{\lambda}\}$ and

$$
l\left(\xi_{1}, \eta_{1}, \xi_{2}, \eta_{2}\right)=\max \left\{\eta_{i}-h^{s}\left(\xi_{i}, \varepsilon\right): i=1,2\right\} .
$$

Proof. For $\left(\xi_{i}, \eta_{i}\right) \in \Omega^{+}\left(\delta_{4}, \rho, \varepsilon\right), i=1,2$, let

$$
\begin{gathered}
\tilde{\xi}(s, \varepsilon)=(1-s) \xi_{1}+s \xi_{2}, \quad 0 \leq s \leq 1, \\
\tilde{\eta}(s, \varepsilon)=\eta_{1}-h^{s}\left(\xi_{1}, \varepsilon\right)+h^{s}(\tilde{\xi}(s, \varepsilon), \varepsilon), \quad 0 \leq s \leq 1 .
\end{gathered}
$$

Then

$$
\begin{gathered}
(\tilde{\xi}(0, \varepsilon), \tilde{\eta}(0, \varepsilon))=\left(\xi_{1}, \eta_{1}\right), \\
(\tilde{\xi}(1, \varepsilon), \tilde{\eta}(1, \varepsilon))=\left(\xi_{2}, \eta_{1}-h^{s}\left(\xi_{1}, \varepsilon\right)+h^{s}\left(\xi_{2}, \varepsilon\right)\right), \\
\tilde{\eta}(s, \varepsilon)-h^{s}(\tilde{\xi}(s, \varepsilon), \varepsilon)=\eta_{1}-h^{s}\left(\xi_{1}, \varepsilon\right), \quad 0 \leq s \leq 1 .
\end{gathered}
$$

Thus, $(\tilde{\xi}(s, \varepsilon), \tilde{\eta}(s, \varepsilon)) \in \Omega^{+}\left(\delta_{4}, \rho, \varepsilon\right)$ for $0 \leq s \leq 1$. Let

$$
\tilde{\tilde{\xi}}(s, \varepsilon)=\xi_{2}, \quad \tilde{\tilde{\eta}}(s, \varepsilon)=(1-s) \tilde{\eta}(1, \varepsilon)+s \eta_{2} .
$$

Then

$$
\begin{gathered}
(\tilde{\tilde{\xi}}(0, \varepsilon), \tilde{\tilde{\eta}}(0, \varepsilon))=\left(\xi_{2}, \tilde{\eta}(1, \varepsilon)\right)=(\tilde{\xi}(1, \varepsilon), \tilde{\eta}(1, \varepsilon)), \\
(\tilde{\tilde{\xi}}(1, \varepsilon), \tilde{\tilde{\eta}}(1, \varepsilon))=\left(\xi_{2}, \eta_{2}\right), \\
\tilde{\tilde{\eta}}(s, \varepsilon)-h^{s}(\tilde{\xi}(s, \varepsilon), \varepsilon)=(1-s)\left(\eta_{1}-h^{s}\left(\xi_{1}, \varepsilon\right)\right) \\
+s\left(\eta_{2}-h^{s}\left(\xi_{2}, \varepsilon\right)\right), \quad 0 \leq s \leq 1 .
\end{gathered}
$$


Thus,

$$
0<\tilde{\tilde{\eta}}(s, \varepsilon)-h^{s}(\tilde{\tilde{\xi}}(s, \varepsilon), \varepsilon)<\rho, \quad 0 \leq s \leq 1,
$$

and

$$
(\tilde{\tilde{\xi}}(s, \varepsilon), \tilde{\tilde{\eta}}(s, \varepsilon)) \in \Omega^{+}\left(\delta_{4}, \rho, \varepsilon\right), \quad 0 \leq s \leq 1
$$

Next,

$$
\begin{aligned}
\left|x_{1}\left(\xi_{1}, \eta_{1}, \varepsilon\right)-x_{1}\left(\xi_{2}, \eta_{2}, \varepsilon\right)\right|_{\alpha} & =\left|x_{1}\left(\xi_{1}, \eta_{1}, \varepsilon\right)-x_{1}\left(\xi_{2}, \tilde{\eta}(1, \varepsilon), \varepsilon\right)+x_{1}\left(\xi_{2}, \tilde{\eta}(1, \varepsilon), \varepsilon\right)-x_{1}\left(\xi_{2}, \eta_{2}, \varepsilon\right)\right| \\
= & \left|\int_{0}^{1} \frac{d}{d s} x_{1}(\tilde{\xi}(s, \varepsilon), \tilde{\eta}(s, \varepsilon), \varepsilon) d s+\frac{d}{d s} x_{1}(\tilde{\xi}(s, \varepsilon), \tilde{\tilde{\eta}}(s, \varepsilon), \varepsilon) d s\right|_{\alpha} \\
\leq & \left|\int_{0}^{1} D_{(\xi, \eta)} x_{1}(\tilde{\xi}(s, \varepsilon), \tilde{\eta}(s, \varepsilon), \varepsilon) \frac{d}{d s}(\tilde{\xi}(s, \varepsilon), \tilde{\eta}(s, \varepsilon)) d s\right|_{\alpha} \\
& +\left|\int_{0}^{1} D_{(\xi, \eta)} x_{1}(\tilde{\xi}(s, \varepsilon), \tilde{\tilde{\eta}}(s, \varepsilon), \varepsilon) \frac{d}{d s}(\tilde{\tilde{\xi}}(s, \varepsilon), \tilde{\tilde{\eta}}(s, \varepsilon)) d s\right|_{\alpha} \\
= & I_{1}+I_{2} .
\end{aligned}
$$

By using the chain rule, Lemmas $1.3,1.4$ and 1.5 and $\rho<\delta_{4} / 4-C_{1} \delta_{4}^{2}$, we have

$$
\begin{aligned}
\mid D_{(\xi, \eta)} & \left.x_{1}(\xi, \eta, \varepsilon)\right|_{\alpha} \\
= & \mid \frac{d}{d t} x(\tau(\xi, \eta, \varepsilon) ; \xi, \eta, \varepsilon) D_{(\xi, \eta)} \tau(\xi, \eta, \varepsilon) \\
& +\left.\left.D_{(\xi, \eta)} x(t ; \xi, \eta, \varepsilon)\right|_{t=\tau(\xi, \eta, \varepsilon)}\right|_{\alpha} \\
\leq & \left|\frac{C_{3}}{\tau(\xi, \eta, \varepsilon)} e^{-\mu \tau(\xi, \eta, \varepsilon)} \frac{8}{\lambda \delta_{4}-8 C_{1} \delta_{4}^{2}} e^{\tilde{\lambda} \tau(\xi, \eta, \varepsilon)}\right|+\left|2 e^{-\tilde{\mu} \tau(\xi, \eta, \varepsilon)}\right| \\
\leq & \frac{8 \tilde{\lambda} C_{3}\left(\delta_{4} / 4\right)^{(-\mu+\tilde{\lambda}) / \tilde{\lambda}}}{-\ln \left(1-4 C_{1} \delta_{4}\right) \cdot\left(\lambda \delta_{4}-8 C_{1} \delta_{4}^{2}\right)}\left|\eta-h^{s}(\xi, \varepsilon)\right|^{(\mu-\tilde{\lambda}) / \tilde{\lambda}} \\
& +2\left(\frac{\delta_{4}}{4}\right)^{-\tilde{\mu} / \tilde{\lambda}}\left|\eta-h^{s}(\xi, \varepsilon)\right|^{\tilde{\mu} / \tilde{\lambda}} .
\end{aligned}
$$

Furthermore, by (1.11), (1.42) and (1.43) we have

$$
\begin{aligned}
\left|\frac{d}{d s}(\tilde{\xi}(s, \varepsilon), \tilde{\eta}(s, \varepsilon))\right|_{\alpha} & =\left|\xi_{1}-\xi_{2}\right|_{\alpha}+\left|D_{x} h^{s}(\tilde{\xi}(s, \varepsilon), \varepsilon)\right|\left|\frac{d}{d s} \tilde{\xi}(s, \varepsilon)\right|_{\alpha} \\
& \leq\left(1+C_{1} \delta_{4}\right)\left|\xi_{1}-\xi_{2}\right|_{\alpha}
\end{aligned}
$$

and

$$
\begin{aligned}
\left|\frac{d}{d s}(\tilde{\xi}(s, \varepsilon), \tilde{\eta}(s, \varepsilon))\right|_{\alpha} & =\left|\eta_{2}-\tilde{\eta}(1, \varepsilon)\right| \leq\left|\eta_{2}-\eta_{1}\right|+\left|h^{s}\left(\xi_{1}, \varepsilon\right)-h^{s}\left(\xi_{2}, \varepsilon\right)\right| \\
& \leq\left(1+C_{1} \delta_{4}\right)\left(\left|\xi_{1}-\xi_{2}\right|_{\alpha}+\left|\eta_{1}-\eta_{2}\right|\right) .
\end{aligned}
$$


Therefore, if we let

$$
\begin{gathered}
\tilde{C}_{4}\left(\delta_{4}\right)=\frac{8 \tilde{\lambda} C_{3}\left(1+C_{1} \delta_{4}\right) \delta_{4}^{(-\mu+\tilde{\lambda}) / \tilde{\lambda}}}{-\ln \left(1-4 C_{1} \delta_{4}\right) \cdot\left(\lambda \delta_{4}-8 C_{1} \delta_{4}^{2}\right)}+2\left(\frac{\delta_{4}}{4}\right)^{-\tilde{\mu} / \tilde{\lambda}} \\
l\left(\xi_{1}, \eta_{1}, \xi_{2}, \eta_{2}\right)=\max \left\{\eta_{i}-h^{s}\left(\xi_{i}, \varepsilon\right): i=1,2\right\} \\
a=\min \{(\mu-\tilde{\lambda}) / \tilde{\lambda}, \tilde{\mu} / \tilde{\lambda}\}
\end{gathered}
$$

then we have

$$
\begin{aligned}
I_{1} & \leq \widetilde{C}_{4}\left(\delta_{4}\right) \int_{0}^{1}\left|\tilde{\eta}(s, \varepsilon)-h^{s}(\tilde{\xi}(s, \varepsilon), \varepsilon)\right|^{a} d s \\
& =\widetilde{C}_{4}\left(\delta_{4}\right)\left|\eta_{1}-h^{s}\left(\xi_{1}, \varepsilon\right)\right|^{a} \\
& \leq \widetilde{C}_{4}\left(\delta_{4}\right) l\left(\xi_{1}, \eta_{1}, \xi_{2}, \eta_{2}\right)^{a}\left(\left|\xi_{1}-\xi_{2}\right|_{\alpha}+\left|\eta_{1}-\eta_{2}\right|\right)
\end{aligned}
$$

Similarly,

$$
\begin{aligned}
I_{2} & \leq \widetilde{C}_{4}\left(\delta_{4}\right) \int_{0}^{1}\left|\tilde{\tilde{\eta}}(s, \varepsilon)-h^{s}(\tilde{\tilde{\xi}}(s, \varepsilon), \varepsilon)\right|^{a} d s \\
& \leq \widetilde{C}_{4}\left(\delta_{4}\right) l\left(\xi_{1}, \eta_{1}, \xi_{2}, \eta_{2}\right)^{a}\left(\left|\xi_{1}-\xi_{2}\right|_{\alpha}+\left|\eta_{1}-\eta_{2}\right|\right) .
\end{aligned}
$$

Hence,

$$
\begin{aligned}
& \left|x_{1}\left(\xi_{1}, \eta_{1}, \varepsilon\right)-x_{1}\left(\xi_{2}, \eta_{2}, \varepsilon\right)\right|_{\alpha} \\
& \quad \leq 2 \widetilde{C}_{4}\left(\delta_{4}\right) l\left(\xi_{1}, \eta_{1}, \xi_{2}, \eta_{2}\right)^{a}\left(\left|\xi_{1}-\xi_{2}\right|_{\alpha}+\left|\eta_{1}-\eta_{2}\right|\right) .
\end{aligned}
$$

Next, by (1.11) and (1.39) we have

$$
\begin{aligned}
& \left|y_{1}\left(\xi_{1}, \eta_{1}, \varepsilon\right)-y_{1}\left(\xi_{2}, \eta_{2}, \varepsilon\right)\right| \\
& =\left|y\left(\tau\left(\xi_{1}, \eta_{1}, \varepsilon\right) ; \xi_{1}, \eta_{1}, \varepsilon\right)-y\left(\tau\left(\xi_{2}, \eta_{2}, \varepsilon\right) ; \xi_{2}, \eta_{2}, \varepsilon\right)\right| \\
& =\mid \delta_{4} / 2+y\left(\tau\left(\xi_{1}, \eta_{1}, \varepsilon\right) ; \xi_{1}, h^{s}\left(\xi_{1}, \varepsilon\right), \varepsilon\right) \\
& \quad-\delta_{4} / 2+y\left(\tau\left(\xi_{2}, \eta_{2}, \varepsilon\right) ; \xi_{2}, h^{s}\left(\xi_{2}, \varepsilon\right), \varepsilon\right) \mid \\
& =\mid h^{s}\left(x\left(\tau\left(\xi_{1}, \eta_{1}, \varepsilon\right) ; \xi_{1}, h^{s}\left(\xi_{1}, \varepsilon\right), \varepsilon\right), \varepsilon\right) \\
& \quad-h^{s}\left(x\left(\tau\left(\xi_{2}, \eta_{2}, \varepsilon\right) ; \xi_{2}, h^{s}\left(\xi_{2}, \varepsilon\right), \varepsilon\right), \varepsilon\right) \mid \\
& \leq C_{1} \delta_{4} \mid x\left(\tau\left(\xi_{1}, \eta_{1}, \varepsilon\right) ; \xi_{1}, h^{s}\left(\xi_{1}, \varepsilon\right), \varepsilon\right) \\
& \quad-\left.x\left(\tau\left(\xi_{2}, \eta_{2}, \varepsilon\right) ; \xi_{2}, h^{s}\left(\xi_{2}, \varepsilon\right), \varepsilon\right)\right|_{\alpha} .
\end{aligned}
$$

Similarly, we can show

$$
\begin{aligned}
& \left|y_{1}\left(\xi_{1}, \eta_{1}, \varepsilon\right)-y_{1}\left(\xi_{2}, \eta_{2}, \varepsilon\right)\right| \\
& \quad \leq 2 C_{1} \delta_{4} \widetilde{C}_{4}\left(\delta_{4}\right) l\left(\xi_{1}, \eta_{1}, \xi_{2}, \eta_{2}\right)^{a}\left(\left|\xi_{1}-\xi_{2}\right|_{\alpha}+\left|\eta_{1}-\eta_{2}\right|\right) .
\end{aligned}
$$

Finally, if we let $C_{4}\left(\delta_{4}\right)=2\left(1+C_{1} \delta_{4}\right) \widetilde{C}_{4}\left(\delta_{4}\right)$, then we have

$$
\begin{gathered}
\left|x_{1}\left(\xi_{1}, \eta_{1}, \varepsilon\right)-x_{1}\left(\xi_{2}, \eta_{2}, \varepsilon\right)\right|_{\alpha}+\left|y_{1}\left(\xi_{1}, \eta_{1}, \varepsilon\right)-y_{1}\left(\xi_{2}, \eta_{2}, \varepsilon\right)\right| \\
\leq C_{4}\left(\delta_{4}\right) l\left(\xi_{1}, \eta_{1}, \xi_{2}, \eta_{2}\right)^{a}\left(\left|\xi_{1}-\xi_{2}\right|_{\alpha}+\left|\eta_{1}-\eta_{2}\right|\right) .
\end{gathered}
$$


In the following, we let $\delta_{4}$ and $\rho<\delta_{4} / 4-C_{1} \delta_{4}^{2}$ be fixed and let

$$
\begin{gathered}
\rho_{0} \leq \min \left\{\left[2^{a+2}\left(1+C_{1} \delta_{4}\right) C_{4}\left(\delta_{4}\right)\right]^{-1 / a}, \rho / 2\right\}, \\
B\left(\rho_{0}\right)=\left\{(\xi, \eta):|\xi|_{\alpha}<\rho_{0},|\eta|<\rho_{0}\right\}, \\
S\left(\delta_{4}, \varepsilon\right)=\left\{(\xi, \eta): \eta=h^{s}(\xi, \varepsilon)+\delta_{4} / 2,|x|_{\alpha}<\delta_{4} / 2\right\} .
\end{gathered}
$$

Since $W_{\text {loc }}^{S}(\varepsilon)$ and $\Omega\left(\delta_{4}, \rho, \varepsilon\right)$ vary continuously in $\varepsilon \in\left[-\varepsilon_{3}, \varepsilon_{3}\right]$, there exists small $0<\varepsilon_{4}<\varepsilon_{3}$ such that for $\rho_{0}$ as in (1.44)

$$
\begin{array}{cc}
B\left(\rho_{0}\right) \cap W_{\mathrm{loc}}^{s}(\varepsilon) \neq \varnothing, & \varepsilon \in\left[-\varepsilon_{4}, \varepsilon_{4}\right], \\
B\left(\rho_{0}\right) \subset \Omega\left(\delta_{4}, \rho, \varepsilon\right), & \varepsilon \in\left[-\varepsilon_{4}, \varepsilon_{4}\right] .
\end{array}
$$

We define a map

$$
\tilde{\pi}^{1}: B\left(\rho_{0}\right) \times\left[-\varepsilon_{4}, \varepsilon_{4}\right] \rightarrow X^{\alpha} \times \mathbf{R}
$$

by

$$
\tilde{\pi}^{1}(\xi, \eta, \varepsilon)=\left\{\begin{array}{l}
\left(x_{1}(\xi, \eta, \varepsilon), y_{1}(\xi, \eta, \varepsilon)\right), \quad \text { if }(\xi, \eta) \in \Omega^{+}\left(\delta_{4}, \rho, \varepsilon\right) \cap B\left(\rho_{0}\right), \\
\left(0, \delta_{4} / 2\right), \quad \text { if }(\xi, \eta) \in\left\{\Omega\left(\delta_{4}, \rho, \varepsilon\right)-\Omega^{+}(\delta, \rho, \varepsilon)\right\} \cap B\left(\rho_{0}\right),
\end{array}\right.
$$

where $\left(x_{1}(\xi, \eta, \varepsilon), y_{1}(\xi, \eta, \varepsilon)\right)$ is as in Lemma 1.6. Note that for each $\varepsilon \in$ $\left[-\varepsilon_{4}, \varepsilon_{4}\right], \tilde{\pi}^{1}(\cdot, \cdot, \varepsilon)$ maps $B\left(\rho_{0}\right)$ into $S\left(\delta_{4}, \varepsilon\right)$ as in (1.45). This definition of $\tilde{\pi}^{1}$ is based on Sil' nikov's ideas in [9 and 10].

Let $Y$ be a Banach space, $f: Y \rightarrow Y$ be a map and $l: Y \times Y \rightarrow \mathbf{R}$ be a realvalued function. In the following lemma and sections, we say $f$ is Lipschitz continuous with Lipschitz constant $l\left(y_{1}, y_{2}\right)$ if

$$
\left|f\left(y_{1}\right)-f\left(y_{2}\right)\right| \leq l\left(y_{1}, y_{2}\right)\left|y_{1}-y_{2}\right|
$$

for every $\left(y_{1}, y_{2}\right) \in Y \times Y$. We note that the "constant" $l$ is dependent on $y_{1}$ and $y_{2}$. This definition will let us have more precise estimates later.

Lemma 1.7. $\tilde{\pi}^{1}(\xi, \eta, \varepsilon)$ is continuous in $(\xi, \eta, \varepsilon) \in B\left(\rho_{0}\right) \times\left[-\varepsilon_{4}, \varepsilon_{4}\right]$ and is Lipschitz continuous in $(\xi, \eta) \in B\left(\rho_{0}\right)$ with Lipschitz constant

$$
C_{5}\left(\delta_{4}\right) l\left(\xi_{1}, \eta_{1}, \xi_{2}, \eta_{2}\right)^{a}
$$

independent of $\varepsilon \in\left[-\varepsilon_{4}, \varepsilon_{4}\right]$, where

$$
\begin{gathered}
C_{5}\left(\delta_{4}\right)=2\left(1+C_{1} \delta_{4}\right) C_{4}\left(\delta_{4}\right), \\
l\left(\xi_{1}, \eta_{1}, \xi_{2}, \eta_{2}\right)=\max \left\{\left|\eta_{i}-h^{s}\left(\xi_{i}, \varepsilon\right)\right|: i=1,2\right\},
\end{gathered}
$$

and $C_{4}\left(\delta_{4}\right)$ and $a$ are as in Lemma 1.6.

Proof. The continuity of $\tilde{\pi}^{1}$ follows from Lemmas 1.3 and 1.4 and Definition (1.48). By (1.44), the definition of $\tilde{\pi}^{1}$ and Lemma 1.6, it suffices to show that $\left\|\tilde{\pi}^{1}\left(\xi_{1}, \eta_{1}, \varepsilon\right)-\tilde{\pi}^{1}\left(\xi_{2}, \eta_{2}, \varepsilon\right)\right\|_{\alpha} \leq C_{5}\left(\delta_{4}\right) l\left(\xi_{1}, \eta_{1}, \xi_{2}, \eta_{2}\right)^{a}\left(\left|\xi_{1}-\xi_{2}\right|_{\alpha}+\left|\eta_{1}-\eta_{2}\right|\right)$ 
holds for those $\left(\xi_{1}, \eta_{1}\right)$ and $\left(\xi_{2}, \eta_{2}\right)$ satisfying $\Delta_{1}=\eta_{1}-h^{s}\left(\xi_{1}, \varepsilon\right)>0$ and $\Delta_{2}=\eta_{2}-h^{s}\left(\xi_{2}, \varepsilon\right) \leq 0$, where $\|\cdot\|_{\alpha}$ is the norm $|\cdot|_{\alpha}+|\cdot|$ in $X^{\alpha} \times \mathbf{R}$.

Let

$$
\begin{aligned}
\tilde{\eta}_{2}(s)=s\left(h^{s}\left(\xi_{2}, \varepsilon\right)+\Delta_{1}\right) & +(1-s) \eta_{2}, \quad 0 \leq s \leq 1 . \\
\left\|\tilde{\pi}^{1}\left(\xi_{1}, \eta_{1}, \varepsilon\right)-\tilde{\pi}^{1}\left(\xi_{2}, \eta_{2}, \varepsilon\right)\right\|_{\alpha} \leq & \left\|\tilde{\pi}^{1}\left(\xi_{1}, \eta_{1}, \varepsilon\right)-\tilde{\pi}^{1}\left(\xi_{2}, \tilde{\eta}_{2}(1), \varepsilon\right)\right\|_{\alpha} \\
& +\left\|\tilde{\pi}^{1}\left(\xi_{2}, \tilde{\eta}_{2}(1), \varepsilon\right)-\tilde{\pi}^{1}\left(\xi_{2}, \eta_{2}, \varepsilon\right)\right\|_{\alpha} \\
= & I_{1}+I_{2} .
\end{aligned}
$$

Because $\tilde{\eta}_{2}(1)-h^{s}\left(\xi_{2}, \varepsilon\right)=\Delta_{1}>0,\left(\xi_{2}, \tilde{\eta}_{2}(1)\right) \in \Omega^{+}\left(\delta_{4}, \rho, \varepsilon\right)$. By Lemma 1.6,

$$
I_{1} \leq C_{4}\left(\delta_{4}\right) l\left(\xi_{1}, \eta_{1}, \xi_{2}, \eta_{2}\right)^{a}\left(\left|\xi_{1}-\xi_{2}\right|_{\alpha}+\left|\eta_{1}-\tilde{\eta}_{2}(1)\right|\right) \text {. }
$$

Since $\left|\eta_{1}-\tilde{\eta}_{2}(1)\right|=\left|h^{s}\left(\xi_{1}, \varepsilon\right)-h^{s}\left(\xi_{2}, \varepsilon\right)\right| \leq C_{1} \delta_{4}\left|\xi_{1}-\xi_{2}\right|_{\alpha}$ by $(1.11)$, so

$$
I_{1} \leq\left(1+C_{1} \delta_{4}\right) C_{4}\left(\delta_{4}\right) l\left(\xi_{1}, \eta_{1}, \xi_{2}, \eta_{2}\right)^{a}\left|\xi_{1}-\xi_{2}\right|_{\alpha}
$$

Note that $\tilde{\eta}_{2}(1)-h^{s}\left(\xi_{2}, \varepsilon\right)=\Delta_{1}>0$, but $\tilde{\eta}_{2}(0)-h^{s}\left(\xi_{2}, \varepsilon\right)=\eta_{2}-h^{s}\left(\xi_{2}, \varepsilon\right)=$ $\Delta_{2} \leq 0$. So there exists $0 \leq \tilde{s} \leq 1$ such that $\tilde{\eta}_{2}(\tilde{s})=h^{s}\left(\xi_{2}, \varepsilon\right)$ and $\tilde{\eta}_{2}(s)-$ $h^{s}\left(\xi_{2}, \varepsilon\right)>0$ for $\tilde{s}<s \leq 1$, implying $\left(\xi_{2}, \tilde{\eta}_{2}(s)\right) \in \Omega^{+}\left(\delta_{4}, \rho, \varepsilon\right)$ for $\tilde{s}<s \leq 1$. Hence, by Lemma 1.6,

$$
\begin{aligned}
& \left\|\tilde{\pi}^{1}\left(\xi_{2}, \tilde{\eta}_{2}(1), \varepsilon\right)-\tilde{\pi}^{1}\left(\xi_{2}, \tilde{\eta}_{2}(s), \varepsilon\right)\right\|_{\alpha} \\
& \quad \leq C_{4}\left(\delta_{4}\right) l\left(\xi_{1}, \eta_{1}, \xi_{2}, \eta_{2}\right)^{a}\left|\tilde{\eta}_{2}(1)-\tilde{\eta}_{2}(s)\right|
\end{aligned}
$$

for $\tilde{s}<s \leq 1$. By the continuity of $\pi^{1}(\xi, \eta, \varepsilon)$ in $(\xi, \eta)$ uniformly in $\varepsilon \in$ $\left[-\varepsilon_{4}, \varepsilon_{4}\right]$ and $(1.46)$, we have

$$
\begin{aligned}
\left\|\tilde{\pi}^{1}\left(\xi_{2}, \tilde{\eta}_{2}(1), \varepsilon\right)-\tilde{\pi}^{1}\left(\xi_{2}, \tilde{\eta}_{2}(\tilde{s}), \varepsilon\right)\right\|_{\alpha} \\
\quad \leq C_{4}\left(\delta_{4}\right) l\left(\xi_{1}, \eta_{1}, \xi_{2}, \eta_{2}\right)^{a}\left|\tilde{\eta}_{2}(1)-\tilde{\eta}_{2}(\tilde{s})\right| \\
\quad=C_{4}\left(\delta_{4}\right) l\left(\xi_{1}, \eta_{1}, \eta_{2}, \eta_{2}\right)^{a}\left|(1-\tilde{s})\left[\left(h^{s}\left(\xi_{2}, \varepsilon\right)-h^{s}\left(\xi_{1}, \varepsilon\right)\right)+\left(\eta_{1}-\eta_{2}\right)\right]\right| \\
\quad \leq\left(1+C_{1} \delta_{4}\right) C_{4}\left(\delta_{4}\right) l\left(\xi_{1}, \eta_{1}, \xi_{2}, \eta_{2}\right)^{a}\left(\left|\xi_{1}-\xi_{2}\right|_{\alpha}+\left|\eta_{1}-\eta_{2}\right|\right) .
\end{aligned}
$$

By the definition of $\tilde{\pi}^{1}$ in $(1.45), \tilde{\pi}^{1}\left(\xi_{2}, \tilde{\eta}_{2}(\tilde{s}), \varepsilon\right)=\tilde{\pi}^{1}\left(\xi_{2}, \eta_{2}, \varepsilon\right)=\left(0, \delta_{4} / 2\right)$. Hence, we have

$$
I_{2} \leq\left(1+C_{1} \delta_{4}\right) C_{4}\left(\delta_{4}\right) l\left(\xi_{1}, \eta_{1}, \xi_{2}, \eta_{2}\right)^{a}\left(\left|\xi_{1}-\xi_{2}\right|_{\alpha}+\left|\eta_{1}-\eta_{2}\right|\right) .
$$

Finally,

$$
\begin{aligned}
& \left\|\tilde{\pi}^{1}\left(\xi_{1}, \eta_{1}, \varepsilon\right)-\tilde{\pi}^{1}\left(\xi_{2}, \eta_{2}, \varepsilon\right)\right\|_{\alpha} \\
& \quad \leq 2\left(1+C_{1} \delta_{4}\right) C_{4}\left(\delta_{4}\right) l\left(\xi_{1}, \eta_{1}, \xi_{2}, \eta_{2}\right)^{\alpha}\left(\left|\xi_{1}-\xi_{2}\right|_{\alpha}+\left|\eta_{1}-\eta_{2}\right|\right) .
\end{aligned}
$$

This proves the lemma.

\section{HOMOCLINIC BIFURCATIONS}

Consider the evolution equation in $X^{\alpha} \times \mathbf{R}$ with a parameter $\varepsilon \in \mathbf{R}$,

$$
\dot{x}=-A x+f(x, y, \varepsilon), \quad \dot{y}=\lambda y+g(x, y, \varepsilon) .
$$


In this section, we will consider the behavior of solutions near a homoclinic orbit of $(2.1)$ which is asymptotic to $(x, y)=(0,0)$ at $\varepsilon=0$. We note that the $C^{3}$ local change of variables (1.6) is valid locally at the origin for equation (2.1). This says that the estimates obtained in $\S 2$ are true for the new variables and the map $\tilde{\pi}^{1}$ is well defined in the new variables. In terms of original variables, it is not clear that similar estimates in Lemma 1.3 will hold. However, the map $\tilde{\pi}^{1}$ is well defined in the original variables and is Lipschitz with a small Lipschitz constant since $\tilde{\pi}^{1}$ is just a map and the local change of variables is $C^{3}$ and is near the identity map. We also note that the domain of the map $\tilde{\pi}^{1}$ is also given by the original variables.

Consider the following hypothesis:

(H3) At $\varepsilon=0$, equation (2.1) has a homoclinic orbit $\Gamma_{0}$ asymptotic to the equilibrium $(0,0)$.

Remark. In fact, hypothesis (H3) implies that $\Gamma_{0}$ belongs to $X^{\alpha} \times \mathbf{R}$.

In this section, we consider the behavior of solutions of equation (2.1) near $\Gamma_{0}$ for $|\varepsilon| \ll 1$. To do this, we consider certain return maps near $\Gamma_{0}$.

(i) Construction of the map $\pi^{1}$. Recall from $\S 1$ that under hypotheses $(\mathrm{H} 1)$ and $(\mathrm{H} 2)$, the local stable and unstable manifolds of the origin $(0,0)$ exist and they are given by (1.2), (1.3) in $\S 1$ :

$$
\begin{aligned}
& W_{\text {loc }}^{s}(\varepsilon)=\left\{(x, y): y=h^{s}(x, \varepsilon),|x|_{\alpha}<\delta_{1}\right\}, \\
& W_{\text {loc }}^{u}(\varepsilon)=\left\{(x, y): x=h^{u}(y, \varepsilon),|y|<\delta_{1}\right\},
\end{aligned}
$$

where $\delta_{1}>0$ is as in $\S 1$ and $h^{s}$ and $h^{u}$ are $C^{3}$ and satisfy (1.4), (1.5). Note that the change of variables $H(\cdot, \cdot, \varepsilon)$ of $(1.6)$ is near the identity map. In the new coordinate system it has been shown in Lemma 1.7 that the map $\tilde{\pi}^{1}: B\left(\rho_{0}\right) \times\left[-\varepsilon_{4}, \varepsilon_{4}\right] \rightarrow X^{\alpha} \times \mathbf{R}$ is well defined by $(1.46)$ where $\rho_{0}, \varepsilon_{4}$ are as in Lemma 1.7. Define

$$
\begin{array}{ll}
G\left(\rho_{0}, \varepsilon\right)=H^{-1}\left(B\left(\rho_{0}\right), \varepsilon\right), & \varepsilon \in\left[-\varepsilon_{4}, \varepsilon_{4}\right], \\
\Sigma\left(\delta_{4}, \varepsilon\right)=H^{-1}\left(S\left(\delta_{4}, \varepsilon\right), \varepsilon\right), & \varepsilon \in\left[-\varepsilon_{4}, \varepsilon_{4}\right],
\end{array}
$$

where $S\left(\delta_{4}, \varepsilon\right)$ is as in (1.45). Since $G\left(\rho_{0}, \varepsilon\right)$ is open, there exists $\rho_{1}$ satisfying

$$
\begin{gathered}
\rho_{1}<\rho_{0} /\left(2+C_{1} \delta_{4}\right), \\
B\left(\rho_{1}\right) \subset \overline{B\left(\rho_{1}\right)} \subset G\left(\rho_{0}, 0\right),
\end{gathered}
$$

where $\overline{B\left(\rho_{1}\right)}$ is the closure of $B\left(\rho_{1}\right)$ in $X^{\alpha} \times \mathbf{R}$. Since both $W_{\text {loc }}^{s}(\varepsilon)$ and $G\left(\rho_{0}, \varepsilon\right)$ vary continuously in $\varepsilon \in\left[-\varepsilon_{4}, \varepsilon_{4}\right]$, there exists a small $0<\varepsilon_{5}<\varepsilon_{4}$ such that the corresponding properties (1.46) and (1.47) in $\S 1$ hold for $B\left(\rho_{1}\right)$, i.e.,

$$
\begin{aligned}
& B\left(\rho_{1}\right) \cap W_{\text {loc }}^{s}(\varepsilon) \neq \varnothing, \quad \varepsilon \in\left[-\varepsilon_{5}, \varepsilon_{5}\right], \\
& B\left(\rho_{1}\right) \subset G\left(\rho_{0}, \varepsilon\right), \quad \varepsilon \in\left[-\varepsilon_{5}, \varepsilon_{5}\right] .
\end{aligned}
$$


Note that by (1.6) and (1.11),

$$
\begin{aligned}
& \left\|D_{(\xi, \eta)} H(\xi, \eta, \varepsilon)\right\|_{\alpha} \leq 2+C_{1} \delta_{4}, \quad(\xi, \eta, \varepsilon) \in B\left(\rho_{1}\right) \times\left[-\varepsilon_{5}, \varepsilon_{5}\right], \\
& \left\|D_{(\xi, \eta)} H^{-1}(\xi, \eta, \varepsilon)\right\|_{\alpha} \leq 2+C_{1} \delta_{4}, \quad(\xi, \eta, \varepsilon) \in B\left(\rho_{1}\right) \times\left[-\varepsilon_{5}, \varepsilon_{5}\right] .
\end{aligned}
$$

Thus, $H(\cdot, \cdot, \varepsilon)$ maps $B\left(\rho_{1}\right)$ into $B\left(\left(2+C_{1} \delta_{4}\right) \rho_{1}\right)$ for $\varepsilon \in\left[-\varepsilon_{5}, \varepsilon_{5}\right]$. By (2.4) we have

$$
H\left(B\left(\rho_{1}\right), \varepsilon\right) \subset B\left(\left(2+C_{1} \delta_{4}\right) \rho_{1}\right) \subset B\left(\rho_{0}\right), \quad \varepsilon \in\left[-\varepsilon_{5}, \varepsilon_{5}\right] .
$$

Define $H \times I: B\left(\rho_{1}\right) \times\left[-\varepsilon_{5}, \varepsilon_{5}\right] \rightarrow X^{\alpha} \times \mathbf{R}$ by $(H \times I)(\xi, \eta, \varepsilon)=(H(\xi, \eta, \varepsilon), \varepsilon)$, and $\tilde{\pi}^{1} \times I: B\left(\rho_{0}\right) \times\left[-\varepsilon_{5}, \varepsilon_{5}\right] \rightarrow X^{\alpha} \times \mathbf{R}$ by $\left(\tilde{\pi}^{1} \times I\right)(\xi, \eta, \varepsilon)=\left(\tilde{\pi}^{1}(\xi, \eta, \varepsilon), \varepsilon\right)$. Then $\tilde{\tilde{\pi}}^{1}: B\left(\rho_{1}\right) \times\left[-\varepsilon_{5}, \varepsilon_{5}\right] \rightarrow X^{\alpha} \times \mathbf{R}$ is well defined, where

$$
\tilde{\tilde{\pi}}^{1}=H^{-1}\left(\left(\tilde{\pi}^{1} \times I\right) \circ(H \times I), \cdot\right) \text {. }
$$

By Lemma 1.7, (2.8) and (2.9), it is clear that $\tilde{\pi}^{1}$ is continuous and Lipschitz continuous in $(\xi, \eta)$ with a Lipschitz constant

$$
\left(2+C_{1} \delta_{4}\right)^{2} C_{5}\left(\delta_{4}\right) l\left(\bar{\xi}_{1}, \bar{\eta}_{1}, \bar{\xi}_{2}, \bar{\eta}_{2}\right)^{a},
$$

where $C_{5}\left(\delta_{4}\right), l$ and $a$ are as in Lemma 1.7 , and $\left(\bar{\xi}_{i}, \bar{\eta}_{i}\right)=H\left(\xi_{i}, \eta_{i}, \varepsilon\right)$ and $\left(\xi_{i}, \eta_{i}\right) \in B\left(\rho_{1}\right), i=1,2, \varepsilon \in\left[-\varepsilon_{5}, \varepsilon_{5}\right]$.

Next, let $p^{0}=\left(\xi^{0}, \eta^{0}\right) \in B\left(\rho_{1}\right) \cap \Gamma_{0}$ be fixed. For every

$$
0<\rho<\operatorname{dist}\left(p^{0}, \partial \overline{B\left(\rho_{1}\right)}\right),
$$

the distance from $p^{0}$ to the boundary of $\overline{B\left(\rho_{1}\right)}$, there exists $0<\varepsilon_{6}(\rho)<\varepsilon_{5}$ such that for $B\left(p^{0}, \rho\right)=\left\{(\xi, \eta):\left|\xi-\xi^{0}\right|_{\alpha}<\rho,\left|\eta-\eta^{0}\right|<\rho\right\}$ it satisfies

$$
B\left(p^{0}, \rho\right) \cap W_{\mathrm{loc}}^{s}(\varepsilon) \neq \varnothing, \quad \varepsilon \in\left[-\varepsilon_{6}(\rho), \varepsilon_{6}(\rho)\right] .
$$

Since $H(\cdot, \cdot, \varepsilon)$ maps $B\left(p^{0}, \rho\right)$ into $B\left(H\left(\xi^{0}, \eta^{0}, \varepsilon\right),\left(2+C_{1} \delta_{4}\right) \rho\right)$ for every $\varepsilon \in\left[-\varepsilon_{6}(\rho), \varepsilon_{6}(\rho)\right]$,

$$
\begin{aligned}
& \sup \left\{l\left(\bar{\xi}_{1}, \bar{\eta}_{1}, \bar{\xi}_{2}, \bar{\eta}_{2}\right):\left(\bar{\xi}_{i}, \bar{\eta}_{i}\right)=H\left(\xi_{i}, \eta_{i}, \varepsilon\right),\left(\xi_{i}, \eta_{i}, \varepsilon\right)\right. \\
& \left.\in B\left(\rho^{0}, \rho\right) \times\left[-\varepsilon_{6}(\rho),-\varepsilon_{6}(\rho)\right], i=1,2\right\} \\
& \leq 2 \sqrt{2}\left(2+C_{1} \delta_{4}\right) \rho,
\end{aligned}
$$

where function $l$ is defined in Lemma 1.7.

Finally, we define $\pi^{1}$ to be the restriction of $\tilde{\tilde{\pi}}^{1}$ to $B\left(p^{0}, \rho\right) \times\left[-\varepsilon_{6}(\rho), \varepsilon_{6}(\rho)\right]$ where $\tilde{\tilde{\pi}}^{1}$ is as in (2.10). In other words, $\pi^{1}$ is defined by

$$
\left(\xi^{1}, \eta^{1}\right)=\pi^{1}(\xi, \eta, \varepsilon)=\tilde{\tilde{\pi}}^{1}(\xi, \eta, \varepsilon), \quad(\xi, \eta, \varepsilon) \in B\left(p^{0}, \rho\right) \times\left[-\varepsilon_{6}(\rho), \varepsilon_{6}(\rho)\right] .
$$

Define

$$
p^{1}(\varepsilon)=\left(\xi^{1}(\varepsilon), \eta^{1}(\varepsilon)\right)=H^{-1}\left(\left(0, \delta_{4} / 2\right), \varepsilon\right), \quad \varepsilon \in\left[-\varepsilon_{6}(\rho), \varepsilon_{6}(\rho)\right] .
$$

It is clear that $p^{1}(\varepsilon)$ is the only intersection point of $W_{\text {loc }}^{u}(\varepsilon)$ with $\Sigma\left(\delta_{4} / 2, \varepsilon\right)$. 
We may summarize the properties of $\pi^{1}$ in the following:

Lemma 2.1. Let $\pi^{1}: B\left(p^{0}, \rho\right) \times\left[-\varepsilon_{6}(\rho), \varepsilon_{6}(\rho)\right] \rightarrow X^{\alpha} \times \mathbf{R}$ be defined by (2.14), where $0<\rho<\operatorname{dist}\left(p^{0}, \partial \overline{B\left(\rho_{1}\right)}\right)$ and $B\left(p^{0}, \rho\right), \varepsilon_{6}(\rho)$ satisfy (2.12). Then $\pi^{1}$ satisfies the following.

(a) Let $(\xi, \eta) \in B\left(p^{0}, \rho\right), \varepsilon \in\left[-\varepsilon_{6}(\rho), \varepsilon_{6}(\rho)\right]$. If $\eta>h^{s}(\xi, \varepsilon)$, then $\pi^{1}(\xi, \eta, \varepsilon)$ is the intersection point of $\Sigma\left(\delta_{4} / 2, \varepsilon\right)$ with the orbit of the solution of $(2.1)$ with initial value $(\xi, \eta)$ and parameter $\varepsilon$. On the other hand, if $\eta \leq h^{s}(\xi, \varepsilon)$, then $\pi^{1}(\xi, \eta, \varepsilon)=\left(\xi^{1}(\varepsilon), \eta^{1}(\varepsilon)\right)$, where $\left(\xi^{1}(\varepsilon), \eta^{1}(\varepsilon)\right)$ is defined by (2.13),

(b) $\pi^{1}(\xi, \eta, \varepsilon)$ is Lipschitzian in $(\xi, \eta) \in B\left(p^{0}, \rho\right)$ with Lipschitz constant $C_{6}\left(\delta_{4}\right) \rho^{a}$ uniformly in $\varepsilon \in\left[-\varepsilon_{6}(\rho), \varepsilon_{6}(\rho)\right]$, where

$$
C_{6}\left(\delta_{4}\right)=2^{3 a / 2}\left(2+C_{1} \delta_{4}\right)^{2+a} C_{5}\left(\delta_{4}\right),
$$

$C_{5}\left(\delta_{4}\right)$ and $a$ are as in Lemma 1.7.

Proof. (a) follows from the definitions of $\tilde{\pi}^{1}$ and $\pi^{1}$.

(b) follows from (2.11) and (2.13).

(ii) Construction of the map $\pi^{2}$. Let $p^{0}$ and $p^{1}(\varepsilon)$ be as in Lemma 2.1 and (2.15) respectively. Since $p^{0}, p^{1}(0)$ are on $\Gamma_{0}$ and $p^{1}(0) \in W_{\text {loc }}^{u}(0)$, there exists $T>0$ such that

$$
\left(x\left(T ; \xi^{1}(0), \eta^{1}(0), 0\right), y\left(T ; \xi^{1}(0), \eta^{1}(0), 0\right)\right)=\left(\xi^{0}, \eta^{0}\right)=p^{0} .
$$

By the continuous dependence property on the initial values and parameter for solutions of equation (2.1) (see Henry [8, pp . 62-70]), there exist constants $r_{1}>0$ and $\varepsilon_{7}>0$ such that the mapping

$$
(x(T ; \cdot, \cdot, \cdot), y(T ; \cdot, \cdot, \cdot)): B\left(p^{1}(0), r_{1}\right) \times\left[-\varepsilon_{7}, \varepsilon_{7}\right] \rightarrow B\left(\rho_{1}\right)
$$

is $C^{3}$, where $B\left(\rho_{1}\right)$ is as in (2.6) and (2.7). We define

$$
\pi^{2}: B\left(p^{1}(0), r_{1}\right) \times\left[-\varepsilon_{7}, \varepsilon_{7}\right] \rightarrow B\left(\rho_{1}\right)
$$

by $\pi^{2}=(x(T ; \cdot, \cdot, \cdot), y(T ; \cdot, \cdot, \cdot))$. Since $\pi^{2}$ is $C^{3}$ at $\left(p^{1}(0), 0\right)$, there exist $0<r_{2}<r_{1}$ and $0<\varepsilon_{8}<\varepsilon_{7}$ such that

$$
\left\|D_{(\xi, \eta, \varepsilon)} \pi^{2}\left(\xi^{1}, \eta^{1}, \varepsilon\right)\right\|_{\alpha} \leq\left\|D_{(\xi, \eta, \varepsilon)} \pi^{2}\left(p^{1}(0), 0\right)\right\|_{\alpha}+1
$$

for every $\left(\xi^{1}, \eta^{1}, \varepsilon\right) \in B\left(p^{1}(0), r_{2}\right) \times\left[-\varepsilon_{8}, \varepsilon_{8}\right]$. Let

$$
C_{7}=\left\|D_{(\xi, \eta, \varepsilon)} \pi^{2}\left(p^{1}(0), 0\right)\right\|_{\alpha}+1 \text {. }
$$

Note that for every $0<r \leq r_{2}$ and $0<\varepsilon \leq \varepsilon_{8}, \pi^{2}$ maps $B\left(p^{1}(0), r\right) \times[-\varepsilon, \varepsilon]$ into $B\left(p^{0}, C_{7} r\right)$ and $\pi^{2}$ is Lipschitzian with Lipschitz constant $C_{7}$.

(iii) Construction of the map $\pi$. Let $p^{1}(\varepsilon)=\left(\xi^{1}(\varepsilon), \eta^{1}(\varepsilon)\right)$ be as in (2.15). Since $p^{1}(\varepsilon)$ is continuous in $\varepsilon \in\left[-\varepsilon_{6}(\rho), \varepsilon_{6}(\rho)\right]$ for every

$$
0<\rho<\operatorname{dist}\left(p^{0}, \partial \overline{B\left(\rho_{1}\right)}\right),
$$


there exists $0<\varepsilon_{9}(\rho)<\varepsilon_{6}(\rho)$ such that

$$
\begin{aligned}
& \left|\xi^{1}(\varepsilon)-\xi^{1}(0)\right|_{\alpha}<C_{6}\left(\delta_{4}\right) \rho^{a} \rho, \quad \varepsilon \in\left[-\varepsilon_{9}(\rho), \varepsilon_{9}(\rho)\right], \\
& \left|\eta^{1}(\varepsilon)-\xi^{1}(0)\right|<C_{6}\left(\delta_{4}\right) \rho^{a} \rho, \quad \varepsilon \in\left[-\varepsilon_{9}(\rho), \varepsilon_{9}(\rho)\right],
\end{aligned}
$$

where $\varepsilon_{6}(\rho), C_{6}\left(\delta_{4}\right)$ and $a$ are as in Lemma 2.1. Let

$$
\begin{aligned}
& \rho_{2}=\min \left\{\left[\frac{1}{2 C_{7} C_{6}\left(\delta_{4}\right)}\right]^{-1 / a},\left[\frac{r_{2}}{2 C_{6}\left(\delta_{4}\right)}\right]^{-1 / a+1}, \operatorname{dist}\left(p^{0}, \partial B\left(\rho_{1}\right)\right)\right\}, \\
& \varepsilon_{10}=\min \left\{\varepsilon_{8}, \varepsilon_{9}\left(\rho_{2}\right)\right\},
\end{aligned}
$$

where $r_{2}, C_{7}$ and $\varepsilon_{8}$ are as in (2.16). By Lemma 2.1 and (2.17), $\pi^{1}$ maps $B\left(p^{0}, \rho_{2}\right) \times\left[-\varepsilon_{10}, \varepsilon_{10}\right]$ into $B\left(p^{1}(0), 2 C_{6}\left(\delta_{4}\right) \rho_{2}^{a} \rho_{2}\right)$. By $(1.19), 2 C_{6}\left(\delta_{4}\right) \rho_{2}^{a} \rho_{2} \leq$ $r_{2}$ and $\varepsilon_{10} \leq \varepsilon_{8}$. Hence, by the construction of $\pi^{2}, \pi^{2}$ maps

$$
B\left(p^{1}(0), 2 C_{6}\left(\delta_{4}\right) \rho_{2}^{a} \rho_{2}\right) \times\left[-\varepsilon_{10}, \varepsilon_{10}\right]
$$

into $B\left(p^{0}, 2 C_{7} C_{6}\left(\delta_{4}\right) \rho_{2}^{a} \rho_{2}\right)$. By $(2.18)$ we have $2 C_{7} C_{6}\left(\delta_{4}\right) \rho_{2}^{a}<1 / 2$, thus, the composition

$$
\pi(\cdot, \cdot, \varepsilon)=\pi^{2}\left(\pi^{1}(\cdot, \cdot, \varepsilon), \varepsilon\right)
$$

maps $B\left(p^{0}, \rho_{2}\right) \times\left[-\varepsilon_{10}, \varepsilon_{10}\right]$ into $B\left(p^{0}, \rho_{2} / 2\right)$. Furthermore, it is clear from Lemma 2.1 and (ii) that $\pi$ is continuous in $(\xi, \eta, \varepsilon)$ and $\pi(\cdot, \cdot, \varepsilon)$ is Lipschitz continuous for every given $\varepsilon \in\left[-\varepsilon_{10}, \varepsilon_{10}\right]$. The Lipschitz constant is $C_{6} C_{6}\left(\delta_{4}\right) \rho_{2}^{a}$ which is less than $1 / 2$ by $(2.18)$ and independent of $\varepsilon \in$ $\left[-\varepsilon_{10}, \varepsilon_{10}\right]$. Therefore $\pi(\cdot, \cdot, \varepsilon)$ is a contraction mapping for every $\varepsilon \in\left[-\varepsilon_{10}, \varepsilon_{10}\right]$.

We may summarize the properties of $\pi$ in the following:

Theorem 2.2. (a) $\pi$ is continuous and $\pi(\cdot, \cdot, \varepsilon)$ is a contraction mapping from $B\left(p^{0}, \rho_{2}\right)$ into $B\left(p^{0}, \rho_{2}\right)$ with contraction constant less than $1 / 2$ uniformly in $\varepsilon \in\left[-\varepsilon_{10}, \varepsilon_{10}\right]$.

(b) If $(\xi, \eta) \in B\left(p^{0}, \rho_{2}\right)$ and $\eta>h^{s}(\xi, \varepsilon)$, then $\pi(\xi, \eta, \varepsilon)$ is on the orbit of equation (2.1) containing $(\xi, \eta)$. If $(\xi, \eta)$ satisfies $\eta-h^{s}(\xi, \varepsilon) \leq 0$, then $\pi(\xi, \eta, \varepsilon)$ is a constant map with $\pi(\xi, \eta, \varepsilon)=\pi^{2}\left(p^{1}(\varepsilon), \varepsilon\right)$, where $\pi^{2}$ and $p^{1}(\varepsilon)$ are as in (ii) and (2.15).

(c)

$$
\begin{array}{ll}
B\left(p^{0}, \rho_{2}\right) \cap\left\{(\xi, \eta): \eta-h^{s}(\xi, \varepsilon)>0\right\} \neq \varnothing, & \varepsilon \in\left[-\varepsilon_{10}, \varepsilon_{10}\right], \\
B\left(p^{0}, \rho_{2}\right) \cap\left\{(\xi, \eta): \eta-h^{s}(\xi, \varepsilon) \leq 0\right\} \neq \varnothing, & \varepsilon \in\left[-\varepsilon_{10}, \varepsilon_{10}\right] .
\end{array}
$$

Proof. (a) follows from the construction of $\pi$ as in (2.19).

(b) follows from Lemma 2.1 .

(c) follows from condition (2.12). 
(iv) Homoclinic bifurcation theorem. Let $p^{1}(\varepsilon)=\left(\xi^{1}(\varepsilon), \eta^{1}(\varepsilon)\right)$ be as in (2.15), and $\varepsilon \in\left[-\varepsilon_{10}, \varepsilon_{10}\right]$. Let

$$
p^{2}(\varepsilon)=\left(\xi^{2}(\varepsilon), \eta^{2}(\varepsilon)\right)=\pi^{2}\left(p^{1}(\varepsilon), \varepsilon\right), \quad \varepsilon \in\left[-\varepsilon_{10}, \varepsilon_{10}\right],
$$

$$
\begin{aligned}
& W_{+}^{u}(\varepsilon)=\left\{(\xi, \eta):(\xi, \eta)=\left(x\left(t ; \xi_{0}, \eta_{0}, \varepsilon\right), y\left(t ; \xi_{0}, \eta_{0}, \varepsilon\right)\right)\right. \\
& \left.\quad \text { for some }\left(\xi_{0}, \eta_{0}\right) \in W_{\text {loc }}^{u}(\varepsilon), \eta_{0}-h^{s}\left(\xi_{0}, \varepsilon\right)>0, t \geq 0\right\}, \\
& \qquad \varepsilon \in\left[-\varepsilon_{10}, \varepsilon_{10}\right] .
\end{aligned}
$$

$W_{+}^{u}(\varepsilon)$ is part of the global unstable manifold of $(0,0)$. Note that $p^{2}(\varepsilon) \in$ $B\left(p^{0}, \rho_{2}\right) \cap W_{+}^{u}(\varepsilon)$. We will assume without loss of generality that $\Gamma_{0}=W_{+}^{u}(0)$.

In the following by a neighborhood $N\left(\Gamma_{0}\right)$ of $\Gamma_{0}$ we mean an open subset in $X^{\alpha} \times \mathbf{R}$ which contains $\Gamma_{0} \cup\{(0,0)\}$.

Theorem 2.3. Consider the following semilinear evolution equation with a real parameter $\varepsilon \in\left[-\varepsilon_{0}, \varepsilon_{0}\right], \varepsilon_{0}>0$ :

$$
\begin{aligned}
& \dot{x}=-A x+f(x, y, \varepsilon), \quad(x, y, \varepsilon) \in X^{\alpha} \times \mathbf{R} \times\left[-\varepsilon_{0}, \varepsilon_{0}\right], \\
& \dot{y}=\lambda y+g(x, y, \varepsilon), \quad(x, y, \varepsilon) \in X^{\alpha} \times \mathbf{R} \times\left[-\varepsilon_{0}, \varepsilon_{0}\right] .
\end{aligned}
$$

Suppose (2.22) satisfies:

(H1) $A$ is a sectorial operator with $\operatorname{Re} \sigma(A) \geq \mu>\lambda>0$;

(H2) For some fixed $0 \leq \alpha<1, f \in C^{3}\left[X^{\alpha} \times \mathbf{R} \times\left[-\varepsilon_{0}, \varepsilon_{0}\right], X\right], g \in$ $C^{3}\left[X^{\alpha} \times \mathbf{R} \times\left[-\varepsilon_{0}, \varepsilon_{0}\right], \mathbf{R}\right]$ and

$$
\begin{array}{lll}
f(0,0, \varepsilon)=0, & D_{(x, y)} f(0,0,0)=0, & \varepsilon \in\left[-\varepsilon_{0}, \varepsilon_{0}\right], \\
g(0,0, \varepsilon)=0, & D_{(x, y)} g(0,0,0)=0, & \varepsilon \in\left[-\varepsilon_{0}, \varepsilon_{0}\right] ;
\end{array}
$$

(H3) At $\varepsilon=0$, equation (2.22) has a homoclinic orbit $\Gamma_{0}$ asymptotic to the equilibrium $(0,0)$.

Then there exists a neighborhood $N\left(\Gamma_{0}\right)$ of $\Gamma_{0}$ and $0<\bar{\varepsilon}_{0}<\varepsilon_{0}$ such that $W_{+}^{u}(\varepsilon) \subset N\left(\Gamma_{0}\right)$ and $W_{+}^{u}(\varepsilon) \cap W_{\mathrm{loc}}^{s}(\varepsilon)=\varnothing$ if and only if there exists a periodic orbit in $N\left(\Gamma_{0}\right)$, where $W_{+}^{u}(\varepsilon)$ is the orbit of $(2.22)$ through $\left(\xi_{0}, \eta_{0}\right)$ satisfying $\left(\xi_{0}, \eta_{0}\right) \in W_{\mathrm{loc}}^{u}(\varepsilon)$ and $\Gamma_{0}=W_{+}^{u}(0)$ (see (2.21)). Furthermore, this periodic orbit is unique in $N\left(\Gamma_{0}\right)$ and is exponentially orbitally asymptotically stable.

To prove our main result above, we need the following lemmas.

Lemma 2.4. Let $p^{1}(\varepsilon)$ and $T>0$ be as in (2.15) and (2.16), respectively. Let $\widetilde{\Gamma}_{0}=\left\{(x, y): x=x\left(t ; p^{1}(0), 0\right), y=y\left(t ; p^{1}(0), 0\right), 0 \leq t \leq T\right\}$. Then there exist $\varepsilon_{11}>0$ and neighborhoods $N_{1}$ and $N_{2}$ of $(0,0) \in X^{\alpha} \times \mathbf{R}$ and $\widetilde{\Gamma}_{0}$, respectively, such that

(a) $N\left(\Gamma_{0}\right)=N_{1} \cup N_{2}$ is a neighborhood of the homoclinic orbit $\Gamma_{0}$;

(b) if $\gamma$ is an orbit of equation (2.22) at $\varepsilon \in\left[-\varepsilon_{11}, \varepsilon_{11}\right]$ satisfying $\gamma \cap N_{1} \neq \varnothing$, $\gamma \subseteq N\left(\Gamma_{0}\right)$, then

$$
\eta-h^{s}(\xi, \varepsilon) \geq 0 \text { for every }(\xi, \eta) \in \gamma \cap N_{1},
$$


where $h^{s}$ is the map whose graph is the local stable manifold (see (1.2));

(c) if $(\xi, \eta) \in N_{2}$ and $\varepsilon \in\left[-\varepsilon_{11}, \varepsilon_{11}\right]$, then $(x(t ; \xi, \eta, \varepsilon), y(t ; \xi, \eta, \varepsilon)) \in$ $B\left(p^{0}, \rho_{2}\right)$ for some $t>0$, where $B\left(p^{0}, \rho_{2}\right)$ is as in Theorem 2.2.

Proof. Let $\delta_{4}, \rho$ and $\Omega\left(\delta_{4}, \rho, \varepsilon\right)$ be as in Lemma 1.4, and $\varepsilon_{4}$ and $S\left(\delta_{4}, \varepsilon\right)$ as in Lemma 1.7. Define

$$
\begin{aligned}
& \tilde{N}(\varepsilon)=\left\{(\xi, \eta):|\xi|_{\alpha}<\delta_{4},-\delta_{4} / \zeta<\eta-h^{s}(\xi, \varepsilon)<\delta_{4}\right\}, \\
& \partial \widetilde{N}^{-}(\varepsilon)=\left\{(\xi, \eta):|\xi|_{\alpha} \leq \delta_{4},-\delta_{4} / 4=\eta-h^{s}(\xi, \varepsilon)\right\}
\end{aligned}
$$

for $\varepsilon \in\left[-\varepsilon_{4}, \varepsilon_{4}\right]$. Then

$$
\begin{aligned}
& \tilde{N}(\varepsilon) \supset \Omega\left(\delta_{4}, \rho, \varepsilon\right), \quad \varepsilon \in\left[-\varepsilon_{4}, \varepsilon_{4}\right], \\
& \tilde{N}(\varepsilon) \supset S\left(\delta_{4}, \varepsilon\right), \quad \varepsilon \in\left[-\varepsilon_{4}, \varepsilon_{4}\right] .
\end{aligned}
$$

By Lemma 1.7, for every $(\xi, \eta) \in \widetilde{N}(\varepsilon) \cap \Omega^{-}\left(\delta_{4}, \rho, \varepsilon\right)$ there exists $t>0$ such that $(x(t ; \xi, \eta, \varepsilon), y(t ; \xi, \eta, \varepsilon)) \in \partial \widetilde{N}^{-}(\varepsilon)$ for $\varepsilon \in\left[-\varepsilon_{4}, \varepsilon_{4}\right]$. Let $H$ be as in (1.6) and

$$
N_{1}=H^{-1}(\tilde{N}(0), 0), \quad \partial N_{1}^{-}=H^{-1}\left(\tilde{N}^{-}(0), 0\right) .
$$

Since $H$ is continuous in $\varepsilon$, there exists $0<\varepsilon_{11}<\varepsilon_{10}$ (see Theorem 2.2) such that

$$
\begin{cases}N_{1} \supset H^{-1}\left(\Omega\left(\delta_{4}, \rho, \varepsilon\right), \varepsilon\right) \supset H^{-1}\left(B\left(\rho_{0}\right), \varepsilon\right)=G\left(\rho_{0}, \varepsilon\right) \supset \begin{array}{l}
B\left(p^{0}, \rho_{2}\right), \\
\\
\varepsilon \in\left[-\varepsilon_{11}, \varepsilon_{11}\right], \\
N_{1} \supset H^{-1}\left(S\left(\delta_{4}, \varepsilon\right), \varepsilon\right)=\Sigma\left(\delta_{4}, \varepsilon\right), \quad \varepsilon \in\left[-\varepsilon_{11}, \varepsilon_{11}\right],
\end{array}\end{cases}
$$

where $B\left(\rho_{0}\right), G\left(\rho_{0}, \varepsilon\right), \Sigma\left(\delta_{4}, \varepsilon\right)$ and $B\left(p^{0}, \rho_{2}\right)$ are as in (2.2), (2.3) and Theorem 2.2. This implies the following properties:

$$
\text { if }(\xi, \eta) \in N_{1} \cap H^{-1}\left(\Omega^{-}\left(\delta_{4}, \rho, \varepsilon\right), \varepsilon\right)-W_{\text {loc }}^{s}(\varepsilon) \text { and } \varepsilon \in\left[-\varepsilon_{11}, \varepsilon_{11}\right] \text {, }
$$
then $(x(t ; \xi, \eta, \varepsilon), y(t ; \xi, \eta, \varepsilon))$ will leave $N_{2}$ through $N_{1}^{-}$;

By continuous dependence on initial values and parameters and property (2.28), for every $\tilde{p}=\left(x\left(t ; p^{1}(0), 0\right), y\left(t ; p^{1}(0), 0\right)\right) \in \widetilde{\Gamma}_{0}$ there exist $\tilde{r}=\tilde{r}(t)>$ 0 and $\tilde{\varepsilon}=\tilde{\varepsilon}(t)>0$ such that $B(\tilde{p}, \tilde{r}) \cap \partial N_{1}^{-}=\varnothing$ and if $(\xi, \eta) \in B(\tilde{p}, \tilde{r})$ and $\varepsilon \in[-\tilde{\varepsilon}, \tilde{\varepsilon}]$, then

$$
(x(T-t ; \xi, \eta, \varepsilon), y(T-t ; \xi, \eta, \varepsilon)) \in B\left(p^{0}, \rho_{2}\right) .
$$

Note that $\bigcup_{0 \leq t \leq T} B(\tilde{p}, \tilde{r})$ is an open cover of $\widetilde{\Gamma}_{0}$. Since $\widetilde{\Gamma}_{0}$ is compact, there exists a finite open subcover $N_{2}=\bigcup_{i=0}^{n} B\left(\tilde{p}_{i}, \tilde{r}_{i}\right)$, where

$$
\tilde{p}_{i}=\left(x\left(t_{i} ; p^{2}(0), 0\right), y\left(t_{i} ; p^{2}(0), 0\right)\right),
$$


$r_{i}=\tilde{r}\left(t_{i}\right)$ and $0 \leq t_{0}<t_{1}<\cdots<t_{n} \leq T$. Without loss of generality we may assume $t_{0}=0$ and $\tilde{p}(0)=p^{1}(0)$. Define $\varepsilon_{11}=\min _{0 \leq i \leq n}\left\{\tilde{\varepsilon}\left(t_{i}\right)\right\}$ and $N\left(\Gamma_{0}\right)=N_{1} \cup N_{2}$. We need to show that $N\left(\Gamma_{0}\right)$ and $\varepsilon_{11}$ satisfy the required properties (a), (b) and (c).

It is obvious that $N\left(\Gamma_{0}\right)$ is an open neighborhood of $\Gamma_{0} \cup\{0\}$. This yields (a). Since $N_{2}^{-} \cap \partial N_{1}=\varnothing$ and $\partial N_{1}^{-} \subset \partial N_{1} \subset \partial N\left(\Gamma_{0}\right)$, the boundary of $N\left(\Gamma_{0}\right)$. This and property (2.27) imply (b). (c) follows from the definition of $B(\tilde{p}, \tilde{r})$.

Lemma 2.5. Let $p^{1}(\varepsilon), N_{2}$ and $T$ be as in Lemma 2.4. There exist $0<\bar{\varepsilon}_{0}<$ $\varepsilon_{11}$ and $\bar{r}>0$ such that the following properties hold:

(a) if $(\xi, \eta, \varepsilon) \in B\left(p^{1}(0), \bar{r}\right) \times\left[-\bar{\varepsilon}_{0}, \bar{\varepsilon}_{0}\right]$, then $(x(t ; \xi, \eta, \varepsilon), y(t ; \xi, \eta, \varepsilon)) \in$ $N_{2}$ for all $0 \leq t \leq T$;

(b) if $\varepsilon \in\left[-\bar{\varepsilon}_{0}, \bar{\varepsilon}_{0}\right]$, then $\left\|p^{1}(\varepsilon)-p^{1}(0)\right\|_{\alpha}<\bar{r} / 4$;

(c) if $p^{*}(\varepsilon)=\left(\xi^{*}(\varepsilon), \eta^{*}(\varepsilon)\right)$ is the unique fixed point of the map $\pi$ in Theorem 2.2 and $\varepsilon \in\left[-\bar{\varepsilon}_{0}, \bar{\varepsilon}_{0}\right]$, then

$$
\left\|p^{*}(\varepsilon)-\left(\xi^{*}(\varepsilon), h^{s}\left(\xi^{*}(\varepsilon), \varepsilon\right)\right)\right\|_{\alpha}<\min \left\{C_{7} \bar{r} / 4, \rho_{2}\right\},
$$

where the constants $C_{7}$ and $\rho_{2}$ are given in (2.17) and (2.19), respectively.

Proof. Let $\tilde{r}(t)$ be as in the proof of Lemma 2.4. By Lemma 2.4, if $(\xi, \eta) \in$ $B\left(p^{1}(0), \tilde{r}(0)\right)$ and $\varepsilon \in\left[-\varepsilon_{11}, \varepsilon_{11}\right]$, then $(x(t ; \xi, \eta, \varepsilon), y(t ; \xi, \eta, \varepsilon))$ is defined for all $0 \leq t \leq T$. We claim that there exist $0<\varepsilon_{12}<\varepsilon_{11}$ and $0<\bar{r}<\tilde{r}(0)$ such that for every $(\xi, \eta, \varepsilon) \in B\left(p^{1}(0), \bar{r}\right) \times\left[-\varepsilon_{12}, \varepsilon_{12}\right]$,

$$
(x(t ; \xi, \eta, \varepsilon), y(t ; \xi, \eta, \varepsilon)) \in N_{2}
$$

for every $0 \leq t \leq T$. Suppose the contrary. Then, there exists a sequence $\left\{\left(\xi_{k}, \eta_{k}, \varepsilon_{k}, t_{k}\right)\right\}, k=1,2, \ldots$, such that $\left(x\left(t_{k} ; \xi_{k}, \eta_{k}, \varepsilon_{k}\right), y\left(t_{k} ; \xi_{k}, \eta_{k}, \varepsilon_{k}\right)\right)$ $\in \partial N_{2},\left(\xi_{k}, \eta_{k}, \varepsilon_{k}\right) \rightarrow\left(\xi^{1}(0), \eta^{2}(0), 0\right)$ as $k \rightarrow+\infty$ and $0 \leq t_{k} \leq T$ for every $k=1,2, \ldots$. Since $[0, T]$ is compact, we may assume $t_{k} \rightarrow t_{0} \in[0, T]$ as $k \rightarrow+\infty$. By passing to the limits, we have

$$
\begin{aligned}
\tilde{\Gamma}_{0} \ni & \left(x\left(t_{0} ; \xi^{1}(0), \eta^{1}(0), 0\right), y\left(t_{0} ; \xi^{1}(0), \eta^{1}(0), 0\right)\right) \\
& =\lim _{k \rightarrow \infty}\left(x\left(t_{k} ; \xi_{k}, \eta_{k}, \varepsilon_{k}\right), y\left(t_{k} ; \xi_{k}, \eta_{k}, \varepsilon_{k}\right)\right) \in \partial N_{2} .
\end{aligned}
$$

This contradicts $\widetilde{\Gamma}_{0} \subset N_{2}$. This yields (a). Next, since $p^{*}(\varepsilon)$ and $p^{1}(\varepsilon)$ are continuous in $\varepsilon$, there exists $0<\varepsilon_{13}<\varepsilon_{10}$ such that properties (b) and (c) hold true for $\varepsilon \in\left[-\varepsilon_{13}, \varepsilon_{13}\right]$. Define

$$
\bar{\varepsilon}_{0}=\min \left\{\varepsilon_{10}, \varepsilon_{11}, \varepsilon_{12}, \varepsilon_{13}\right\} .
$$

This yields the desired results.

Lemma 2.6. Let $\bar{\varepsilon}_{0}$ and $p^{*}(\varepsilon)$ be as in Lemma 2.5 and $N\left(\Gamma_{0}\right)$ be the neighborhood in Lemma 2.4. If $\gamma$ is a periodic or homoclinic orbit of equation (2.22) at $\varepsilon \in\left[-\bar{\varepsilon}_{0}, \bar{\varepsilon}_{0}\right]$ and $\gamma \subseteq N\left(\Gamma_{0}\right)$, then $p^{*}(\varepsilon) \in \gamma$. 
Proof. Let $\gamma$ be either a homoclinic, or periodic orbit of equation (2.22) at $\varepsilon \in$ $\left[-\bar{\varepsilon}_{0}, \bar{\varepsilon}_{0}\right]$. We will show that the inclusion $\gamma \subset N\left(\Gamma_{0}\right)$ implies $p^{*}(\varepsilon) \in \gamma$, where $p^{*}(\varepsilon)$ is the unique fixed point of the map $\pi(\cdot, \cdot, \varepsilon)$ defined in Theorem 2.2. If $\gamma$ is a homoclinic orbit, then $p^{1}(\varepsilon) \in \gamma$ and $\tilde{\tilde{p}}(\varepsilon)=\pi^{2}\left(p^{1}(\varepsilon), \varepsilon\right) \in B\left(p^{0}, \rho_{2}\right)$, where $\rho_{2}$ is as in (2.18) and $\varepsilon \in\left[-\bar{\varepsilon}_{0}, \bar{\varepsilon}_{0}\right]$. Since $\gamma \subset N\left(\Gamma_{0}\right)$, then $\gamma \cap N_{1} \cap$ $H^{-1}\left(\Omega^{-}\left(\delta_{4}, \rho, \varepsilon\right), \varepsilon\right)=\varnothing$ by Lemma $2.4(\mathrm{~b})$. By Theorem 2.2(b), $(\pi)^{k}(\tilde{\tilde{p}}(\varepsilon), \varepsilon)$ is on $\gamma$ for every $k=0,1,2, \ldots$, where $(\pi)^{k}(\tilde{\tilde{p}}(\varepsilon), \varepsilon)=\pi\left((\pi)^{k-1}(\tilde{\tilde{p}}(\varepsilon), \varepsilon), \varepsilon\right)$ is the $k$ th iterate of $\pi(\cdot, \cdot, \varepsilon)$. Since $\gamma \cap W_{\text {loc }}^{s}(\varepsilon) \neq \varnothing$, there exists $K \geq 0$ such that $(\pi)^{K}(\tilde{\tilde{p}}(\varepsilon), \varepsilon) \in W_{\mathrm{loc}}^{s}(\varepsilon)$. By Theorem 2.2, $\pi\left((\pi)^{K}(\tilde{\tilde{p}}(\varepsilon), \varepsilon), \varepsilon\right)=$ $\pi^{2}\left(p^{1}(\varepsilon), \varepsilon\right)=\tilde{\tilde{p}}(\varepsilon)$, i.e., $p(\pi)^{K+1}(\tilde{\tilde{p}}(\varepsilon), \varepsilon)=\tilde{\tilde{p}}(\varepsilon)$. Thus, $\tilde{\tilde{p}}(\varepsilon)$ is a fixed point of $(\pi)^{K+1}(\cdot, \cdot, \varepsilon)$. Since every fixed point of any given iteration of a contraction mapping is a fixed point of itself, we have $\tilde{\tilde{p}}(\varepsilon)=p^{*}(\varepsilon)$. Similarly, if $\gamma$ is a periodic orbit in $N\left(\Gamma_{0}\right)$, by Lemma $2.4(\mathrm{~b}), \gamma \cap H^{-1}\left(\Omega^{-}\left(\delta_{4}, \rho, \varepsilon\right), \varepsilon\right)=\varnothing$. By the hyperbolicity of $(0,0) \in N_{1}, \gamma \not \subset N_{1}$, i.e., $\gamma \cap N_{2} \neq \varnothing$. By Lemma 2.4(c), there must be a point $q^{0} \in \gamma \cap B\left(p^{0}, \rho_{0}\right)$. Since $\gamma \cap H^{-1}\left(\Omega^{-}\left(\delta_{4}, \rho, \varepsilon\right), \varepsilon\right)=\varnothing$, by Theorem 2.2(b), $q^{k}=(\pi)^{k+1}\left(q^{0}, \varepsilon\right)$ is on $\gamma$ for every $k=0,1,2, \ldots$. Since $\gamma$ is periodic, there exists $K \geq 0$ with $q^{K}=q^{1}$, i.e. $q^{1}=(\pi)^{K}\left(q^{1}, \varepsilon\right)$. This implies that $q^{1}=q^{*}(\varepsilon)$ by the uniqueness of the fixed point for $(\pi)^{k}(\cdot, \cdot, \varepsilon)$, $k=1,2, \ldots$.

Proof of Theorem 2.3. We show that the condition in the theorem is necessary. Suppose $W_{+}^{u}(\varepsilon) \subset N\left(\Gamma_{0}\right)$ for some $\varepsilon \in\left[-\bar{\varepsilon}_{0}, \bar{\varepsilon}_{0}\right]$, then by Lemma 2.4 (c) $W_{+}^{u}(\varepsilon) \cap H^{-1}\left(\Omega^{-}\left(\delta_{4}, \rho, \varepsilon\right), \varepsilon\right)=\varnothing$. We claim that

$$
p^{*}(\varepsilon) \notin H^{-1}\left(\Omega^{-}\left(\delta_{4}, \rho, \varepsilon\right), \varepsilon\right) .
$$

Suppose the contrary. By Theorem 2.2(b), $p^{*}(\varepsilon)=\pi\left(p^{*}(\varepsilon), \varepsilon\right)=\pi^{2}\left(p^{1}(\varepsilon), \varepsilon\right) \in$ $W_{+}^{u}(\varepsilon)$. This is a contradiction. Since $p^{*}(\varepsilon) \notin H^{-1}\left(\Omega^{-}\left(\delta_{4}, \rho, \varepsilon\right), \varepsilon\right)$, by Theorem $2.2(\mathrm{~b}), \pi\left(p^{*}(\varepsilon), \varepsilon\right)=p^{*}(\varepsilon)$ is on an orbit $\gamma$ of a solution of equation (2.22). It follows that $\gamma$ is a periodic orbit. Next, we need to show $\gamma \subset N\left(\Gamma_{0}\right)$. Let the real valued function $\tau$ be as in Lemma 1.4 and

$$
\tau^{*}(\varepsilon)=\tau\left(H\left(p^{*}(\varepsilon), \varepsilon\right), \varepsilon\right) .
$$

Then, by Lemma 1.4, $\left(x\left(t ; p^{*}(\varepsilon), \varepsilon\right), y\left(t ; p^{*}(\varepsilon), \varepsilon\right)\right) \in N_{1}$ for $0 \leq t \leq \tau^{*}(\varepsilon)$. By (2.18), Lemma 2.1 and Lemma 2.5(b), (c) we have

$$
\begin{aligned}
\left\|\pi^{1}\left(p^{*}(\varepsilon), \varepsilon\right)-p^{1}(0)\right\|_{\alpha} & \leq\left\|\pi^{1}\left(p^{*}(\varepsilon), \varepsilon\right)-p^{1}(\varepsilon)\right\|_{\alpha}+\left\|p^{1}(\varepsilon)-p^{1}(0)\right\|_{\alpha} \\
& \leq C_{6}\left(\delta_{4}\right) \rho_{2}^{a} \min \left\{C_{7} \bar{r} / 4, \rho_{2}\right\}+\bar{r} / 4 \\
& \leq \bar{r} / 4+\bar{r} / 4=\bar{r} / 2,
\end{aligned}
$$

where $C_{6}\left(\delta_{4}\right) \quad a, \rho_{2}$ and $C_{7}$ are as in Lemma 2.1 and Theorem $2.2 ; \bar{r}$ is as in Lemma 2.5. Thus, by Lemma $2.4(\mathrm{c})$,

$$
\left(x\left(t+\tau^{*}(\varepsilon) ; p^{*}(\varepsilon), \varepsilon\right), y\left(t+\tau^{*}(\varepsilon) ; p^{*}(\varepsilon), \varepsilon\right)\right) \in N_{2}
$$


for $0 \leq t \leq T$. Therefore $\gamma \subset N\left(\Gamma_{0}\right)$. We note that the period $\omega^{*}(\varepsilon)$ of $\gamma$ is $\omega^{*}(\varepsilon)=\tau^{*}(\varepsilon)+T$.

We now show that the condition in the theorem is also sufficient. Let $\gamma$ be a periodic orbit. By Lemma 2.6, $p^{*}(\varepsilon) \in \gamma$. Let $\tilde{\tilde{p}}(\varepsilon)=\pi^{2}\left(p^{1}(\varepsilon), \varepsilon\right) \in$ $W_{+}^{u}(\varepsilon) \cap B\left(p^{0}, \rho_{2}\right)$ be as in the proof of Lemma 2.6 and $\tau$ be as in Lemma 1.4. Define

$$
\begin{aligned}
\tilde{\tilde{p}}^{k}(\varepsilon) & =(\pi)^{k}(\tilde{\tilde{p}}(\varepsilon), \varepsilon), \quad \varepsilon \in\left[-\bar{\varepsilon}_{0}, \bar{\varepsilon}_{0}\right], \\
\tilde{\tilde{\tau}}^{k}(\varepsilon) & =\tau\left(H\left(\tilde{\tilde{p}}^{k}(\varepsilon), \varepsilon\right), \varepsilon\right), \quad \varepsilon \in\left[-\bar{\varepsilon}_{0}, \bar{\varepsilon}_{0}\right],
\end{aligned}
$$

for $k=1,2, \ldots$. We will show that

$$
\tilde{\tilde{p}}^{k}(\varepsilon) \in H^{-1}\left(\Omega^{+}\left(\delta_{4}, \rho_{2}, \varepsilon\right), \varepsilon\right), \quad \varepsilon \in\left[-\bar{\varepsilon}_{0}, \bar{\varepsilon}_{0}\right], k=1,2, \ldots
$$

and

$$
\begin{aligned}
& \bigcup_{0 \leq t \leq T}\left\{(x, y): x=x\left(t ; p^{1}(\varepsilon), \varepsilon\right), y=y\left(t ; p^{1}(\varepsilon), \varepsilon\right)\right\} \subset N\left(\Gamma_{0}\right), \\
& \bigcup_{0 \leq t \leq \tilde{\tilde{\tau}}^{k}(\varepsilon)+T}\left\{(x, y): x=x\left(t ; \tilde{\tilde{p}}^{k}(\varepsilon), \varepsilon\right), y=y\left(t ; \tilde{\tilde{p}}^{k}(\varepsilon), \varepsilon\right)\right\} \subset N\left(\Gamma_{0}\right), \\
& \\
& \varepsilon \in\left[-\bar{\varepsilon}_{0}, \bar{\varepsilon}_{0}\right], k=1,2 \ldots .
\end{aligned}
$$

Note that if (2.30) and (2.31) hold true, then, given a $p \in W_{+}^{u}(\varepsilon), p$ is in either

$$
\bigcup_{0 \leq t \leq T}\left\{(x, y): x=x\left(t ; p^{1}(\varepsilon), \varepsilon\right), y=y\left(t ; p^{1}(\varepsilon), \varepsilon\right)\right\} \subset N\left(\Gamma_{0}\right)
$$

or

$$
\bigcup_{0 \leq t \leq \tilde{\tau}^{k}(\varepsilon)+T}\left\{(x, y): x=x\left(t ; \tilde{\tilde{p}}^{k}(\varepsilon), \varepsilon\right), y=y\left(t ; \tilde{\tilde{p}}^{k}(\varepsilon), \varepsilon\right)\right\} \cup N\left(\Gamma_{0}\right)
$$

for some $k=1,2, \ldots$ Therefore, the conclusion $W_{+}^{u}(\varepsilon) \subset N\left(\Gamma_{0}\right)$ holds true. Thus it suffices to show (2.30) and (2.31). Suppose (2.30) is not true, then there exists $K$ such that $\tilde{\tilde{p}}^{K}(\varepsilon) \in H^{-1}\left(\Omega^{-}\left(\delta_{4}, \rho_{2}, \varepsilon\right), \varepsilon\right) \cup W_{\text {loc }}^{s}(\varepsilon)$ and $\tilde{\tilde{p}}^{k}(\varepsilon) \notin H^{-1}\left(\Omega^{-}\left(\delta_{4}, \rho_{2}, \varepsilon\right), \varepsilon\right) \cup W_{\mathrm{loc}}^{s}(\varepsilon), k=1,2, \ldots, K-1$. By Theorem 2.2(b), $\pi\left(\tilde{\tilde{p}}^{K}(\varepsilon), \varepsilon\right)=\pi\left((\pi)^{K}(\tilde{\tilde{p}}(\varepsilon), \varepsilon), \varepsilon\right)=\tilde{\tilde{p}}(\varepsilon)$. It follows that $\tilde{\tilde{p}}(\varepsilon)$ is the fixed point of $(\pi)^{K+1}$. Thus, it is the fixed point of $\pi$ and $\tilde{\tilde{p}}(\varepsilon)=p^{*}(\varepsilon) \in \gamma$. This is a contradiction and proves (2.30). By Theorem $2.2(\mathrm{~b}), \tilde{\tilde{p}}^{k}(\varepsilon)$ is on $W_{+}^{u}(\varepsilon)$ for every $k=1,2, \ldots$. By Lemma 2.5 , we have

$$
\bigcup_{0 \leq t \leq T}\left\{(x, y): x=x\left(t ; p^{1}(\varepsilon), \varepsilon\right), y=y\left(t ; p^{1}(\varepsilon), \varepsilon\right)\right\} \subset N\left(\Gamma_{0}\right) .
$$

By Theorem 2.2(a) and Lemma 2.5(b),(c) we have

$$
\begin{aligned}
\left\|\tilde{\tilde{p}}^{k}(\varepsilon)-p^{*}(\varepsilon)\right\|_{\alpha} & \leq\left(\frac{1}{2}\right)^{k}\left\|\tilde{p}^{*}(\varepsilon)-p^{*}(\varepsilon)\right\|_{\alpha} \\
& \leq\left(\frac{1}{2}\right)^{k} C_{7} \bar{r} / 4
\end{aligned}
$$


where $\tilde{p}^{*}(\varepsilon)=\left(\xi^{*}(\varepsilon), h^{s}\left(\xi^{*}(\varepsilon), \varepsilon\right)\right) \in W_{\text {loc }}^{s}(\varepsilon)$. By Lemma 2.1, (2.18) and (2.30) we have

$$
\begin{aligned}
& \left\|\pi^{1}\left(\tilde{\tilde{p}}^{k}(\varepsilon), \varepsilon\right)-\pi^{1}\left(p^{*}(\varepsilon), \varepsilon\right)\right\|_{\alpha} \\
& \quad \leq C_{6}\left(\delta_{4}\right) \rho_{2}^{2}\left\|\tilde{\tilde{p}}^{k}(\varepsilon)-p^{*}(\varepsilon)\right\|_{\alpha} \\
& \quad \leq C_{6}\left(\delta_{4}\right) C_{7} \rho_{2}^{2}(1 / 2)^{k} \bar{\gamma} / 4 \leq \bar{r} / 8 .
\end{aligned}
$$

By (2.29) and (2.33) we have

$$
\left\|\pi^{1}\left(\tilde{\tilde{p}}^{k}(\varepsilon), \varepsilon\right)-p^{1}(0)\right\|_{\alpha}<\bar{r} / 2+\bar{r} / 8<\bar{r} .
$$

It follows that, by Lemma $2.5(\mathrm{a})$,

$$
\bigcup_{\tilde{\tau}^{k}(\varepsilon) \leq t \leq \tilde{\tau}^{k}(\varepsilon)+T}\left\{(x, y): x=x\left(t ; \tilde{\tilde{p}}^{k}(\varepsilon), \varepsilon\right), y=y\left(t ; \tilde{\tilde{p}}^{k}(\varepsilon), \varepsilon\right)\right\} \subset N_{2} .
$$

By the definition of $N_{1}$ and Lemma 1.4 we have

$$
\bigcup_{0 \leq t \leq \bar{\tau}^{*}(\varepsilon)}\left\{(x, y): x=x\left(t ; \tilde{\tilde{p}}^{k}(\varepsilon), \varepsilon\right), y=y\left(t ; \tilde{\tilde{p}}^{k}(\varepsilon), \varepsilon\right)\right\} \subset N_{1} .
$$

Therefore (2.31) is proved. Finally, the exponentially asymptotic stability of $\gamma$ follows from Theorem 2.2(a).

Corollary 2.7. Suppose the same hypotheses of Theorem 2.3 hold for equation (2.22). Then there exists a neighborhood $N\left(\Gamma_{0}\right)$ of $\Gamma_{0} \cup\{(0,0)\}$ in $X^{\alpha} \times \mathbf{R}$ and $0<\bar{\varepsilon}_{0}<\varepsilon_{0}$ such that if there is a homoclinic orbit in $N\left(\Gamma_{0}\right)$ for equation (2.22) at $|\varepsilon| \leq \bar{\varepsilon}_{0}$, then there exist no periodic orbits in $N\left(\Gamma_{0}\right)$.

\section{HOMOCLINIC BIFURCATION FOR RETARDED FUNCTIONAL DIFFERENTIAL EQUATIONS}

In this section, we will derive analogous results of the previous sections for retarded functional differential equations. For the basis theory of retarded functional differential equations, we follow the usual treatment of Hale [7].

Let $\mathbf{R}^{n}$ be the $n$-dimensional Euclidean real space, let $r$ be a fixed positive constant and let $C$ be the space of continuous functions from $[-r, 0]$ into $\mathbf{R}^{n}$ with the usual sup-norm $|\phi|=\sup _{-r \leq \theta \leq 0}|\phi(\theta)|$.

Consider the linear autonomous delay equation

$$
\dot{x}(t)=L\left(x_{t}\right), \quad L(\phi)=\int_{-r}^{0}[d \eta(\theta)] \phi(\theta), \quad \phi \in C,
$$

where $\eta$ is an $n \times n$ matrix function of bounded variation and $x_{t} \in C$ with $x_{t}(\theta)=x(t+\theta)$ for $-r \leq \theta \leq 0$. Consider the perturbed autonomous equation with a real parameter $\varepsilon \in\left[-\varepsilon_{0}, \varepsilon_{0}\right]$ :

$$
\dot{x}(t)=L\left(x_{t}\right)+f\left(x_{t}, \varepsilon\right),
$$

where $f: C \times\left[-\varepsilon_{0}, \varepsilon_{0}\right] \rightarrow \mathbf{R}^{n}$ is continuous and takes bounded sets into bounded sets, $\varepsilon_{0}>0$ is a fixed constant. 
Let $x(\phi)$ and $x(\phi, \varepsilon)$ be the real-valued solutions of (3.1) and (3.2) respectively with initial value $\phi \in C$ at $t=0$. Let $T(t)$ be the semigroup generated by the solution $x_{t}(\phi)$ and let $A$ be its infinitesimal generator with domain $\mathscr{D}(A)=\left\{\phi: \phi \in C^{1}, \phi(0)=L(\phi)\right\}$. The spectrum $\sigma(A)$ of $A$ contains only point spectrum and $\lambda \in \sigma(A)$ if and only if $\lambda$ satisfies the characteristic equation $\operatorname{det} \Delta(\lambda)=0$, where

$$
\Delta(\lambda)=\lambda I-\int_{-r}^{0} e^{\lambda \theta} d \eta(\theta)
$$

Our discussion will be based on the following hypotheses:

(H4) The characteristic equation $\operatorname{det} \Delta(\lambda)=0$ has a unique root $\lambda>0$ which is simple and the real parts of all other solutions of $\operatorname{det} \Delta(\lambda)=0$ are strictly less than $-\lambda$;

(H5) $f(\phi, \varepsilon)$ is $C^{3}$ in $\phi$ and $\varepsilon$ and satisfies

$$
f(0, \varepsilon)=0, \quad \varepsilon \in\left[-\varepsilon_{0}, \varepsilon_{0}\right], \quad D_{\phi} f(0, \varepsilon)=0, \quad \varepsilon \in\left[-\varepsilon_{0}, \varepsilon_{0}\right] .
$$

By hypothesis (H4), $C$ is decomposed by the eigenvalue $\lambda$ of $A$ as $C=$ $S \oplus U$, where $U$ is the one-dimensional eigenspace of $\{\lambda\}$. Let $\varphi_{\lambda}=\varphi_{\lambda}(0) e^{\lambda \theta}$ be the eigenvector of $\lambda$ and $\psi_{\lambda}$ be a row eigenvector corresponding to the eigenvalue $\lambda$ of the formal adjoint operator $A^{*}$ of $A$ associated with the bilinear form

$$
\langle\alpha, \phi\rangle=\alpha(0) \phi(0)-\int_{-r}^{0} \int_{0}^{\theta} \alpha(\xi-\theta) d \eta(\theta) \phi(\xi) d \xi,
$$

where $\alpha$ is a $1 \times n$ continuous row function on $[0, r]$ and $\phi \in C$. It is known that

$$
\begin{aligned}
& U=\left\{\phi: \phi \in C, \phi=a \varphi_{\lambda} \text { for some } a \in \mathbf{R}\right\} \\
& S=\left\{\phi: \phi \in C,\left\langle\psi_{\lambda}, \phi\right\rangle=0\right\} .
\end{aligned}
$$

For every $\phi \in C, \phi=\phi^{s}+\phi^{u}$ with $\phi^{u}=\left\langle\psi_{\lambda}, \phi\right\rangle \varphi_{\lambda} \in U$ and $\phi^{s}=\phi-\phi^{u} \in S$. Note that $U$ and $S$ are closed subspaces of $C$.

Let $X_{0}(\theta)$ be the matrix function defined by

$$
X_{0}(\theta)= \begin{cases}0, & -r \leq \theta<0, \\ I, & \theta=0 .\end{cases}
$$

We may extend the definition of $T(t)$ to include its action on $X_{0}$ (see [7]). $X_{0}$ has the following decomposition:

$$
X_{0}^{u}=\varphi_{\lambda} \psi_{\lambda}(0), \quad X_{0}^{s}=X_{0}-X_{0}^{u} .
$$


Note that if $v \in \mathbf{R}^{n}$, then $T(t) X_{0}^{u} v \in U$ for all $t \in \mathbf{R}$ and $T(t) X_{0}^{s} v \in S$ for $t \geq r$. Furthermore, there exist constants $K_{1}$ and $\mu>\lambda>0$ such that

$$
\begin{aligned}
& \left|T(t) \phi^{s}\right| \leq K_{1} e^{-\mu t}\left|\phi^{s}\right|, \quad t \geq 0, \phi^{s} \in S, \\
& \left|T(t) X_{0}^{s}\right| \leq K_{1} e^{-\mu t}, \quad t \geq 0, \\
& T(t) \phi(0)=T(t+\theta) \phi(0), \quad t+\theta \geq 0, \\
& T(t) \phi(\theta)=\phi(t+\theta), \quad-r \leq t+\theta \leq 0, \\
& T(t) \phi^{u}=\phi^{u} e^{\lambda t}, \quad-\infty<t<+\infty, \phi^{u} \in U, \\
& \phi^{u}(\theta)=\phi^{u}(0) e^{\lambda \theta}, \quad \theta \in[-r, 0], \phi^{u} \in U .
\end{aligned}
$$

Let the solution $x(\phi, \varepsilon)$ of (3.2) be with initial value $\phi$. Then $x_{t}(\phi, \varepsilon)$ can be decomposed as $x_{t}(\phi, \varepsilon)=x_{t}^{s}(\phi, \varepsilon)+x_{t}^{u}(\phi, \varepsilon)$, with $x_{t}^{s}(\phi, \varepsilon) \in S$ and $x_{t}^{u}(\phi, \varepsilon) \in U$. Then we have the variation of constants formula (see [7, pp. 143-147, 185-188]):

$$
\begin{aligned}
& x_{t}^{s}(\phi, \varepsilon)=T(t) \phi^{s}+\int_{0}^{t} T(t-\alpha) X_{0}^{s} f\left(x_{\alpha}(\phi, \varepsilon), \varepsilon\right) d \alpha, \\
& x_{t}^{u}(\phi, \varepsilon)=e^{\lambda t} \phi^{u}+\int_{0}^{t} e^{\lambda(t-\alpha)} X_{0}^{u} f\left(x_{\alpha}(\phi, \varepsilon), \varepsilon\right) d \alpha .
\end{aligned}
$$

Let $P^{s}: C \rightarrow S$ and $P^{u}: C \rightarrow U$ be the projection operators, i.e. if $\phi=$ $\phi^{s}+\phi^{u} \in C$, then $P^{s} \phi=\phi^{s}$ and $P^{u} \phi=\phi^{u}$. Since $f$ is $C^{3}, x_{t}(\phi, \varepsilon)$ is $C^{3}$ in $(\phi, \varepsilon)$ for all $t \geq 0$ in the maximal interval of existence. Let $y_{t}^{s}(\phi, \varepsilon)=$ $D_{\phi} x_{t}^{s}(\phi, \varepsilon)$ and $y_{t}^{u}(\phi, \varepsilon)=D_{\phi} x_{t}^{u}(\phi, \varepsilon)$. It follows from (3.6) that

$$
\begin{aligned}
& y_{t}^{s}(\phi, \varepsilon)=T(t) P^{s}+\int_{0}^{t} T(t-\alpha) X_{0}^{s} D_{\phi} f\left(x_{\alpha}(\phi, \varepsilon), \varepsilon\right) \cdot y_{\alpha}(\phi, \varepsilon) d \alpha, \\
& y_{t}^{u}(\phi, \varepsilon)=T(t) P^{u}+\int_{0}^{t} T(t-\alpha) X_{0}^{u} D_{\phi} f\left(x_{\alpha}(\phi, \varepsilon), \varepsilon\right) \cdot y_{\alpha}(\phi, \varepsilon) d \alpha .
\end{aligned}
$$

Note that if $\phi \in C$, then

$$
T(t) P^{u} \phi=e^{\lambda t} \phi^{u}, \quad t \in \mathbf{R} .
$$

By hypotheses (H4) and (H5), there exist $\delta_{1}>0$ and $0<\varepsilon_{1}<\varepsilon_{0}$ such that the local stable and unstable manifolds $W_{\text {loc }}^{s}(\varepsilon), W_{\text {loc }}^{u}(\varepsilon)$ exist in $B\left(\delta_{1}\right)$ for $\varepsilon \in\left[-\varepsilon_{1}, \varepsilon_{1}\right]$, where $B\left(\delta_{1}\right)=\left\{\phi \in C:\left|\phi^{s}\right|<\delta_{1},\left|\phi^{u}\right|<\delta_{1}\right\}$ (see [7, pp. 210-214]). Moreover,

$$
\begin{aligned}
& W_{\text {loc }}^{s}(\varepsilon)=\left\{\phi=\phi^{s}+\phi^{u}: \phi^{u}=h_{s}\left(\phi^{s}, \varepsilon\right),\left|\phi^{s}\right| \leq \delta_{1}\right\}, \\
& W_{\text {loc }}^{u}(\varepsilon)=\left\{\phi=\phi^{s}+\phi^{u}: \phi^{s}=h_{u}\left(\phi^{u}, \varepsilon\right),\left|\phi^{u}\right| \leq \delta_{1}\right\},
\end{aligned}
$$

where $h_{s}$ and $h_{u}$ are $C^{3} . h_{u}$ is given by

$$
h_{u}\left(\phi^{u}, \varepsilon\right)=\int_{-\infty}^{0} T(-\alpha) X_{0}^{s} f\left(x_{\alpha}^{*}\left(\phi^{u}, \varepsilon\right), \varepsilon\right) d \alpha, \quad\left|\phi^{u}\right| \leq \delta_{1}, \varepsilon \in\left[-\varepsilon_{1}, \varepsilon_{1}\right]
$$


where $x_{t}^{*}\left(\phi^{u}, \varepsilon\right), t \leq 0$, is the unique bounded solution of (3.2) with

$$
\left|x_{t}^{*}\left(\phi^{u}, \varepsilon\right)\right|<K_{2}\left|\phi^{u}\right| e^{\mu t}, \quad t \leq 0,
$$

for some constant $K_{2}$ independent of $\left(\phi^{u}, \varepsilon\right)$, and $h_{u}\left(\phi^{u}, \varepsilon\right)=\left(x_{0}^{*}\left(\phi^{u}, \varepsilon\right)\right)^{s}$, $\phi^{u}=\left(x_{0}^{*}\left(\phi^{u}, \varepsilon\right)\right)^{u}$. Because of hypothesis (H5), we may assume for constant $K_{2}>0$ that the following estimates also hold for $\left|\phi^{s}\right| \leq \delta_{1},\left|\phi^{u}\right| \leq \delta_{1}$ and $\varepsilon \in\left[-\varepsilon_{1}, \varepsilon_{1}\right]$ :

$$
\begin{aligned}
& \left|h_{s}\left(\phi^{s}, \varepsilon\right)\right| \leq K_{2}\left|\phi^{s}\right|^{2}, \\
& \left|D_{\phi^{s}} h_{s}\left(\phi^{s}, \varepsilon\right) \cdot \psi^{s}\right| \leq K_{2}\left|\phi^{s}\right|\left|\psi^{s}\right|, \quad \psi^{s} \in S, \\
& \left|D_{\phi^{s}}^{2} h_{s}\left(\phi^{s}, \varepsilon\right) \cdot\left(\psi_{1}^{s}, \psi_{2}^{s}\right)\right| \leq K_{2}\left|\psi_{1}^{s}\right|\left|\psi_{2}^{s}\right|, \quad \psi_{i}^{s} \in S, \quad i=1,2, \\
& \left|h_{u}\left(\phi^{u}, \varepsilon\right)\right| \leq K_{2}\left|\phi^{u}\right|^{2}, \\
& \left|D_{\phi^{u}} h_{u}\left(\phi^{u}, \varepsilon\right) \cdot \psi^{u}\right| \leq K_{2}\left|\phi^{u}\right|\left|\psi^{u}\right|, \quad \psi^{u} \in U, \\
& \left|D_{\phi^{u}}^{2} h_{u}\left(\phi^{u}, \varepsilon\right) \cdot\left(\psi_{1}^{u}, \psi_{2}^{u}\right)\right| \leq K_{2}\left|\psi_{1}^{u}\right|\left|\psi_{2}^{u}\right|, \quad \psi_{i}^{u} \in U, \quad i=1,2,
\end{aligned}
$$

where $D_{\phi}^{k}$ denotes the $k$ th partial differentiation operator with respect to $\phi$. We will use $D_{\phi}$ for $D_{\phi}^{1}$.

We have the following:

Proposition 3.1. For every $\left|\phi^{u}\right|<\delta_{1}$ and $\varepsilon \in\left[-\varepsilon_{1}, \varepsilon_{1}\right], h_{u}\left(\phi^{u}, \varepsilon\right)(\theta)$ is $C^{4}$ in $\theta \in[-r, 0]$.

Proof. Since $\phi^{u}(\theta)=a \varphi_{\lambda}(0) e^{\lambda \theta}$ for some $a \in \mathbf{R}, \phi^{u}$ is $C^{\infty}$. Since $\phi^{u}+$ $h_{u}\left(\phi^{u}, \varepsilon\right)=\phi$ is an element in the local unstable manifold $W_{\text {loc }}^{u}(\varepsilon)$, for every $t>0$ there exists $\psi \in W_{\text {loc }}^{u}(\varepsilon)$ such that $x_{t}(\psi, \varepsilon)=\phi$. This implies by the smoothing property of the solution operator that $\phi(\theta)$ is $C^{4}$ in $\theta \in[-r, 0]$. Hence, $h_{u}\left(\phi^{u}, \varepsilon\right)(\theta)=\phi(\theta)-\phi^{u}(\theta)$ is $C^{4}$ in $\theta \in[-r, 0]$.

Let $\left|\phi^{u}\right|<\delta_{1}$ and $|\varepsilon| \leq \varepsilon_{1}$. To simplify the notation, we let $x_{t}^{*}=x_{t}^{*}\left(\phi^{u}, \varepsilon\right)$ and $h_{u}=h_{u}\left(\phi^{u}, \varepsilon\right)$. Note that $x_{t}^{*}, h_{u} \in C$. By differentiating (3.9) with respect to $\theta \in[-r, 0]$, we have

$$
\begin{aligned}
\frac{d}{d \theta} h_{u}(\theta)= & X_{0}(0) f\left(x_{\theta}^{*}\right)+L\left(\int_{-\infty}^{0} T(-\alpha) X_{0}^{s} f\left(x_{\alpha+\theta}^{*}, \varepsilon\right) d \alpha\right) \\
& -\int_{\theta}^{0} \frac{d}{d \theta} \varphi_{\lambda}(\theta-\alpha) \psi_{\lambda}(0) f\left(x_{\alpha}^{*}, \varepsilon\right) d \alpha .
\end{aligned}
$$

Since $\frac{d}{d \theta} h_{u}(\theta)$ is a function in $\left(\phi^{u}, \varepsilon\right)$ with values in $\mathbf{R}^{n}, D_{\phi^{u}} \frac{d}{d \theta} h_{u}(\theta)$ is a continuous linear map from $U$ to $\mathbf{R}^{n}$. On the other hand, $\frac{d}{d \theta} h_{u}$ is a function in $\left(\phi^{u}, \varepsilon\right)$ with values in $C$ and $D_{\phi^{u}} \frac{d}{d \theta} h_{u}$ is a continuous map from $U$ into $C$. The following proposition shows that we may interchange the order of differentiations. 
Proposition 3.2. Let $\phi^{u} \in U,\left|\phi^{u}\right|<\delta_{1}, \theta \in[-r, 0]$ and $-\varepsilon_{1} \leq \varepsilon \leq \varepsilon_{1}$. Then

(a)

$$
\frac{d}{d \theta}\left(D_{\phi^{u}} h_{u} \cdot \phi_{1}^{u}\right)(\theta)=\left(D_{\phi^{u}} \frac{d}{d \theta} h_{u}(\theta)\right) \cdot \phi_{1}^{u}, \quad \phi_{1}^{u} \in U
$$

(b)

$$
\frac{d}{d \theta}\left(D_{\phi^{u}}^{2} h_{u} \cdot\left(\phi_{1}^{u}, \phi_{2}^{u}\right)\right)(\theta)=\left(D_{\phi^{u}}^{2} \frac{d}{d \theta} h_{u}(\theta)\right) \cdot\left(\phi_{1}^{u}, \phi_{2}^{u}\right), \quad \phi_{1}^{u}, \phi_{2}^{u} \in U ;
$$

(c)

$$
\frac{d}{d \theta}\left(D_{\phi^{u}} \frac{d}{d \theta} h_{u} \cdot \phi_{1}^{u}\right)(\theta)=\left(D_{\phi^{u}} \frac{d^{2}}{d \theta^{2}} h_{u}(\theta)\right) \cdot \phi_{1}^{u}, \quad \phi_{1}^{u} \in U .
$$

Proof. Let $f_{1}(t)=D_{\phi^{u}}\left[f\left(x_{t}^{*}, \varepsilon\right)\right]$ and $f_{2}(t)=D_{\phi^{u}}^{2}\left[f\left(x_{t}^{*}, \varepsilon\right)\right]$. Then $f_{1}(t): U \rightarrow$ $\mathbf{R}^{n}$ is continuous and linear and $f_{2}(t): U \times U \rightarrow \mathbf{R}^{n}$ is continuous and bilinear.

By (3.9) we have

$$
\begin{gathered}
D_{\phi^{u}} h_{u} \cdot \phi_{1}^{u}=\int_{-\infty}^{0} T(-\alpha) X_{0}^{s} f_{1}(\alpha) \cdot \phi_{1}^{u} d \alpha, \quad \phi_{1}^{u} \in U, \\
D_{\phi^{u}}^{2} h_{u} \cdot\left(\phi_{1}^{u}, \phi_{2}^{u}\right)=\int_{-\infty}^{0} T(-\alpha) X_{0}^{s} f_{2}(\alpha) \cdot\left(\phi_{1}^{u}, \phi_{2}^{u}\right) d \alpha, \quad \phi_{1}^{u}, \phi_{2}^{u} \in U .
\end{gathered}
$$

By direct computation, we have

$$
\begin{aligned}
\frac{d}{d \theta}\left(D_{\phi^{u}} h_{u} \cdot \phi_{1}^{u}\right)(\theta)= & X_{0}(0) f_{1}(\theta) \cdot \phi_{1}^{u}+L\left(\int_{-\infty}^{0} T(-\alpha) X_{0}^{s} f_{2}(\alpha+\theta) \cdot \phi_{1}^{u} d \alpha\right) \\
& -\int_{-\theta}^{0} \frac{d}{d \theta} \varphi_{\lambda}(\theta-\alpha) \psi_{\lambda}(0) f_{1}(\alpha) \cdot \phi_{1}^{u} d \alpha \\
= & \left(D_{\phi^{u}} \frac{d}{d \theta} h_{u}(\theta)\right) \cdot \phi_{1}^{u}, \quad \phi_{1}^{u} \in U,
\end{aligned}
$$

and

$$
\begin{aligned}
\frac{d}{d \theta}\left(D_{\phi^{u}}^{2} h_{u} \cdot\left(\phi_{1}^{u}, \phi_{2}^{u}\right)\right)(\theta)= & X_{0}(0) f_{2}(\theta) \cdot\left(\phi_{1}^{u}, \phi_{2}^{u}\right) \\
& +L\left(\int_{-\infty}^{0} T(-\alpha) X_{0}^{s} f_{2}(\alpha+\theta) \cdot\left(\phi_{1}^{u}, \phi_{2}^{u}\right) d \alpha\right) \\
& -\int_{\theta}^{0} \frac{d}{d \theta} \varphi_{\lambda}(\theta-\alpha) \psi_{\lambda}(0) f_{2}(\alpha) \cdot\left(\phi_{1}^{u}, \phi_{2}^{u}\right) d \alpha \\
= & \left(D_{\phi^{u}}^{2} \frac{d}{d \theta} h_{u}(\theta)\right) \cdot\left(\phi_{1}^{u}, \phi_{2}^{u}\right), \quad \phi_{1}^{u}, \phi_{2}^{u} \in U .
\end{aligned}
$$

This proves (a) and (b). Note that by (3.12) we also have

$$
\left(D_{\phi^{u}} \frac{d}{d \theta} h_{u} \cdot \phi_{1}^{u}\right)(\theta)=\left(D_{\phi^{u}} \frac{d}{d \theta} h_{u}(\theta)\right) \cdot \phi_{1}^{u} .
$$


To show that $\left(D_{\phi^{u}} \frac{d}{d \theta} h_{u} \cdot \phi_{1}^{u}\right)(\theta)$ is $C^{1}$ in $\theta \in[-r, 0]$, it suffices to show by (3.12) that $f_{1}(t)$ is differentiable in $t \leq 0$. Recall

$$
f_{1}(t)=D_{\phi} f\left(x_{t}^{*}, \varepsilon\right) \cdot D_{\phi^{u}} x_{t}^{*}
$$

Since $f$ is $C^{3}$, we only need to show $x_{t}^{*}$ and $D_{\phi^{u}} x_{t}^{*}$ are at least $C^{1}$ in $t$. Since $x^{*}\left(\phi^{u}, \varepsilon\right)$ is a solution of $(3.2)$ and $D_{\phi^{u}} x^{*}\left(\phi^{u}, \varepsilon\right)$ is the solution of the variational equation along $x_{t}^{*}$,

$$
\dot{y}(t)=L\left(y_{t}\right)+D_{\phi} f\left(x_{t}^{*}, \varepsilon\right) \cdot y_{t},
$$

which is defined for $t \leq 0$, we have

$$
\begin{aligned}
\frac{\partial}{\partial t} x_{t}^{*}\left(\phi^{u}, \varepsilon\right) & =\left(\dot{x}^{*}\left(\phi^{u}, \varepsilon\right)\right)_{t}, \\
\frac{\partial}{\partial t} D_{\phi^{u}} x_{t}^{*}\left(\phi^{u}, \varepsilon\right) & =\left(D_{\phi^{u}} \dot{x}^{*}\left(\phi^{u}, \varepsilon\right)\right)_{t},
\end{aligned}
$$

where $\left(\dot{x}^{*}\left(\phi^{u}, \varepsilon\right)\right)_{t}(\theta)=\dot{x}^{*}\left(\phi^{u}, \varepsilon\right)(t+\theta)$. Hence $f_{1}(t)$ is $C^{1}$ in $t \leq 0$. By differentiating (3.11) with respect to $\theta$ and $\phi^{u}$ and then differentiating (3.12) with respect to $\theta$, we obtain (c).

Remark. The smoothness properties of $D_{\phi^{u}} h(\theta), D_{\phi^{u}}^{2} h(\theta)$ and $D_{\phi^{u}} \frac{d}{d \theta} h(\theta)$ in $\theta \in[-r, 0]$ are not optimal in the above proposition.

Corollary 3.3. Under the hypotheses of Proposition 3.2

$$
\frac{d}{d \theta}\left[\left(D_{\phi^{u}}^{2} h_{u}\right) \cdot\left(\phi_{1}^{u}, \phi_{2}^{u}\right)\right] \text { and } \frac{d}{d \theta}\left[\left(D_{\phi^{u}} \frac{d}{d \theta} h^{u}\right) \cdot \phi_{1}^{u}\right]
$$

are elements in $C$ for all $\phi_{1}^{u}, \phi_{2}^{u} \in U$. Furthermore, there exists a constant $K_{3}$ depending on $\delta_{1}, \varepsilon_{1}, K_{1}$ and $K_{2}$ such that

$$
\begin{gathered}
\left|\frac{d}{d \theta}\left[\left(D_{\phi^{u}}^{2} h_{u}\right) \cdot\left(\phi_{1}^{u}, \phi_{2}^{u}\right)\right]\right| \leq K_{3}\left|\phi_{1}^{u}\right|\left|\phi_{2}^{u}\right|, \quad \phi_{1}^{u}, \phi_{2}^{u} \in U ; \\
\left|\frac{d}{d \theta}\left[\left(D_{\phi^{u}} \frac{d}{d \theta} h_{u}\right) \cdot \phi_{1}^{u}\right]\right| \leq K_{3}\left|\phi^{u}\right|\left|\phi_{1}^{u}\right|, \quad \phi_{1}^{u} \in U .
\end{gathered}
$$

Proof. This follows from (b) and (c) of Proposition 3.2, (3.5) and (3.10).

Note that $\left|\phi^{u}\right|$ appears on the right-hand side of the second inequality above. This is because the unstable manifold is tangent to the linear space $U$ at the origin.

Note that by (3.9) we have

$$
\frac{d}{d \theta} h_{u}(0)=L\left(h_{u}\right)+X_{0}(0) f\left(h_{u}+\phi^{u}, \varepsilon\right) .
$$


This says that $h_{u}\left(\phi^{u}, \varepsilon\right) \notin \mathscr{D}(A)$ if $\phi^{u} \neq 0$. However, we have

Proposition 3.4. Let $\phi^{u} \in U,\left|\phi^{u}\right|<\delta_{1}, \theta \in[-r, 0]$ and $|\varepsilon| \leq \varepsilon_{1}$. Then there exists a constant $K_{4}$ depending on $\delta_{1}, \varepsilon_{1}$ and $K_{1}-K_{3}$ such that

(a) $\left.\frac{d}{d t} T(t) h_{u}\right|_{t=0^{+}}=\frac{d}{d \theta} h_{u}-X_{0} f\left(h_{u}+\phi^{u}, \varepsilon\right)$;

(b) $\left.\left|T(t) \frac{d}{d \tau} T(\tau) h_{u}\right|_{\tau=0^{+}}\left|\leq K_{4}\right| \phi^{u}\right|^{2} e^{-\mu t}, t \geq 0$,

where the derivative $\frac{d}{d t}$ is taken in $L^{\infty}$.

Proof. (a) By (3.5) and (3.9) we have

$$
\begin{aligned}
T(t) h_{u}(\theta)= & \int_{-\infty}^{t+\theta}\left[X_{0}^{s}(0)+\int_{0}^{t+\theta-\alpha} L\left(T(\beta) X_{0}^{s}\right) d \beta\right] f\left(x_{\alpha}^{*}, \varepsilon\right) d \alpha \\
& -\int_{t+\theta}^{0} \varphi_{\lambda}(t+\theta-\alpha) \psi_{\lambda}(0) f\left(x_{\alpha}^{*}, \varepsilon\right) d \alpha, \quad t+\theta<0, t>0, \\
T(t) h_{u}(0)= & \int_{-\infty}^{0}\left[X_{o}^{2}(0)+\int_{0}^{t-\alpha} L\left(T(\beta) X_{0}^{s}\right) d \beta\right] f\left(x_{\alpha}^{*}, \varepsilon\right) d \alpha, \quad t>0 .
\end{aligned}
$$

If $\theta<0$, then by (3.11) and by differentiating (3.13) we have

$$
\left.\frac{d}{d t} T(t) h_{u}(\theta)\right|_{t=0^{+}}=\frac{d}{d \theta} h_{u}(\theta)=\frac{d}{d \theta} h_{u}(\theta)-X_{0}(\theta) f\left(h_{u}+\phi^{u}, \varepsilon\right) \text {. }
$$

If $\theta=0$, then by (3.19), (3.11) and (3.13) we have

$$
\begin{aligned}
\left.\frac{d}{d t} T(t) h_{u}(0)\right|_{t=0^{+}} & =\lim _{t \rightarrow 0^{+}} \frac{1}{t}\left[T(t) h_{u}(0)-h_{u}(0)\right] \\
& =\lim _{t+0^{+}} \frac{1}{t} \int_{-\infty}^{0}\left[\int_{-\alpha}^{t-\alpha} L\left(T(\beta) X_{0}^{s}\right) d \beta\right] f\left(x_{\alpha}^{*}, \varepsilon\right) d \alpha \\
& =L\left(\int_{-\infty}^{0} T(-\alpha) X_{0}^{s} f\left(x_{\alpha}^{*}, \varepsilon\right) d \alpha\right) \\
& =\frac{d}{d \theta} h_{u}(0)-X_{0}(0) f\left(h_{u}+\phi^{u}, \varepsilon\right) .
\end{aligned}
$$

This proves (a).

(b) By Proposition 3.1 and (a) above,

$$
\left.T(t) \frac{d}{d \tau} T(\tau) h_{u}\right|_{\tau=0^{+}}
$$

is well defined. Moreover, since $T(t) h_{u} \in S$ is continuous for $t \geq 0$ and differentiable for $t>r$ and $S$ is a closed subspace,

$$
\left.T(t) \frac{d}{d \tau} h_{u}\right|_{\tau=0^{+}}=\frac{d}{d t} T(t) h_{u} \in S, \quad t>r .
$$

It follows from (a) of Proposition 3.2 that

$$
\left.T(t) \frac{d}{d \tau} T(\tau) h_{u}\right|_{\tau=0^{+}}, \quad t \geq 0,
$$


is $C^{3}$ in $\left(\phi^{u}, \varepsilon\right)$. Moreover, since

$$
D_{\phi^{u}} \frac{d}{d \theta} h_{u}(0, \varepsilon)=0, \quad \varepsilon \in\left[-\varepsilon_{1}, \varepsilon_{1}\right]
$$

we have

$$
\left.D_{\phi^{u}} T(t) \frac{d}{d \tau} T(\tau) h_{u}(0, \varepsilon)\right|_{\tau=0^{+}}=0, \quad t \geq 0, \varepsilon \in\left[-\varepsilon_{1}, \varepsilon_{1}\right] .
$$

Therefore, by (3.10) we have

$$
\widetilde{K}=\sup _{\substack{\left|\phi^{u}\right| \leq \delta_{1} \\|\varepsilon| \leq \varepsilon_{1}}} e^{2 \mu r}\left|D_{\phi^{u}}^{2} T(2 r) \frac{d}{d \tau} T(\tau) h_{u}\right|_{\tau=0^{+}} \mid<+\infty,
$$

and $\widetilde{K}$ depends on $\delta_{1}, \varepsilon_{1}, K_{1}, K_{2}$ and $K_{3}$. Thus, by (3.5) we have

$$
\left.\left|T(t) \frac{d}{d \tau} T(\tau) h_{u}\right|_{\tau=0^{+}}\left|\leq K_{1} \tilde{K} e^{-\mu t}\right| \phi^{u}\right|^{2}, \quad t \geq 2 r .
$$

Again by $(3.10)$ we have

$$
\widetilde{\widetilde{K}}=\sup _{\substack{\left|\phi^{u}\right| \leq \delta_{1} \\|\varepsilon| \leq \varepsilon_{1} \\ 0 \leq t \leq 2 r}} e^{2 \mu r}\left|D_{\phi^{u}}^{2} T(t) \frac{d}{d \tau} T(\tau) h_{u}\right|_{\tau=0^{+}} \mid<+\infty,
$$

and $\widetilde{\widetilde{K}}$ depends on $\delta_{1}, \varepsilon_{1}, K_{1}, K_{2}$ and $K_{3}$. Thus,

$$
\left.\left|T(t) \frac{d}{d \tau} T(\tau) h_{u}\right|_{\tau=0^{+}}\left|\leq \widetilde{\widetilde{K}} e^{-\mu t}\right| \phi^{u}\right|^{2}, \quad 0 \leq t \leq 2 r .
$$

Define $K_{4}=\max \left\{\widetilde{K} K_{1}, \widetilde{\widetilde{K}}\right\}$. This proves (b).

Define a change of variables $\bar{\phi}=H(\phi, \varepsilon), \phi \in B\left(\delta_{1}\right),|\varepsilon| \leq \varepsilon_{1}$ by

$$
\bar{\phi}^{s}=\phi^{s}-h_{u}\left(\phi^{u}, \varepsilon\right), \quad \bar{\phi}^{u}=\phi^{u} .
$$

It is clear from (3.10) that $H$ is near the identity and in the new variable $W_{\text {loc }}^{u}(\varepsilon)=\left\{\bar{\phi}: \bar{\phi} \in H\left(B\left(\delta_{1}\right), \varepsilon\right), \bar{\phi}^{s}=0\right\}$. The inverse $H^{-1}$ of $H$ is given by

$$
\phi^{s}=\bar{\phi}^{s}+h_{u}\left(\bar{\phi}^{u}, \varepsilon\right), \quad \phi^{u}=\bar{\phi}^{u} .
$$

Note that since $x_{t}^{u}(\phi, \varepsilon)$ is $C^{3}$ in $(t, \phi, \varepsilon), t \geq 0,|\phi| \leq \delta,|\varepsilon| \leq \varepsilon_{1}$, the composition map $h_{u}\left(x_{t}^{u}(\phi, \varepsilon), \varepsilon\right)$ is $C^{3}$ in $t$ and $(\phi, \varepsilon)$. By this change of variables, (3.6) becomes

$$
\begin{aligned}
\bar{x}_{t}^{s}(\bar{\phi}, \varepsilon)= & T(t) \bar{\phi}^{s}+\int_{0}^{t} T(t-\alpha) X_{0}^{s} \bar{f}\left(\bar{x}_{\alpha}, \varepsilon\right) d \alpha \\
& +T(t) h_{u}\left(\bar{\phi}^{u}, \varepsilon\right)-T(0) h_{u}\left(\bar{x}_{t}^{u}, \varepsilon\right) \\
\bar{x}_{t}^{u}(\bar{\phi}, \varepsilon)= & e^{\lambda t} \bar{\phi}^{u}+\int_{0}^{t} e^{\lambda(t-\alpha)} X_{0}^{u} \bar{f}\left(\bar{x}_{\alpha}, \varepsilon\right) d \alpha
\end{aligned}
$$


where $\bar{x}_{t}=\bar{x}_{t}(\bar{\phi}, \varepsilon)=H\left(x_{t}(\phi, \varepsilon), \varepsilon\right)$ and $\bar{f}$ is defined by

$$
\bar{f}\left(\bar{x}_{\alpha}, \varepsilon\right)=f\left(H^{-1}\left(\bar{x}_{\alpha}, \varepsilon\right), \varepsilon\right) .
$$

Note that in the new variables, $\bar{x}_{t}(\phi, \varepsilon)$ may not satisfy a functional differential equation. However, we can make such a transformation, discuss the behavior of $\bar{x}_{t}(\phi, \varepsilon)$ as a solution of the integral equation and then return to $x_{t}$ to obtain information about the original equation.

By using Propositions 3.2-3.4, (3.15) can be written as

$$
\begin{aligned}
& \bar{x}_{t}^{s}(\bar{\phi}, \varepsilon)=T(t) \bar{\phi}^{s}+\int_{0}^{t} T(t-\alpha) \bar{g}\left(\bar{x}_{\alpha}, \varepsilon\right) d \alpha, \\
& \bar{x}_{t}^{u}(\bar{\phi}, \varepsilon)=e^{\lambda t} \bar{\phi}^{u}+\int_{0}^{t} e^{\lambda(t-\alpha)} X_{0}^{u} \bar{f}\left(\bar{x}_{\alpha}, \varepsilon\right) d \alpha,
\end{aligned}
$$

where $\bar{g}$ is given by

$$
\begin{aligned}
\bar{g}(\bar{\phi}, \varepsilon)= & X_{0}^{s} \bar{f}(\bar{\phi}, \varepsilon)-\left.\frac{d}{d t} T(t) h_{u}\left(\bar{\phi}^{u}, \varepsilon\right)\right|_{t=0^{+}} \\
& -D_{\bar{\phi}^{u}} h_{u}\left(\bar{\phi}^{u}, \varepsilon\right) \cdot\left[\lambda \bar{\phi}^{u}+X_{0}^{u} \bar{f}(\bar{\phi}, \varepsilon)\right] \\
= & X_{0}^{u} \bar{f}(\bar{\phi}, \varepsilon)-\frac{d}{d \theta} h_{u}\left(\bar{\phi}^{u}, \varepsilon\right)-D_{\bar{\phi}^{u}} h_{u}\left(\bar{\phi}^{u}, \varepsilon\right) \cdot\left[\lambda \bar{\phi}^{u}+X_{0}^{u} \bar{f}(\bar{\phi}, \varepsilon)\right] \\
& +X_{0}\left[\bar{f}(\bar{\phi}, \varepsilon)+\bar{f}\left(h_{u}\left(\bar{\phi}^{u}, \varepsilon\right)+\bar{\phi}^{u}, \varepsilon\right)\right] .
\end{aligned}
$$

Note that $X_{0}$ appears in (3.18). This says that $\bar{g}(\bar{\phi}, \varepsilon)$ has a discontinuity at $\theta=0$, hence $\bar{g}(\bar{\phi}, \varepsilon) \in L^{\infty}=L^{\infty}\left([-r, 0], \mathbf{R}^{n}\right)$. Since $H$ is near the identity map, by (3.10) there exist $\delta_{2}>0$ and $0<\varepsilon_{2}<\varepsilon_{1}$ such that

$$
B\left(\delta_{2}\right) \subset H\left(B\left(\delta_{1}\right), \varepsilon\right), \quad|\varepsilon| \leq \varepsilon_{2} .
$$

Therefore, $\bar{g}: B\left(\delta_{2}\right) \times\left[-\varepsilon_{2}, \varepsilon_{2}\right] \rightarrow L^{\infty}$ is $C^{2}$. By (H5), (3.10) and (3.11), $D_{(\bar{\phi}, \varepsilon)} \bar{g}(0, \varepsilon)=0$ for $|\bar{\phi}| \leq \delta_{2}$ and $|\varepsilon| \leq \varepsilon_{2}$. By Proposition 3.2, $\bar{g}(\bar{\phi}, \varepsilon)(\theta)$ and $D_{\bar{\phi}} \bar{g}(\bar{\phi}, \varepsilon)(\theta)$ are actually differentiable in $\theta \in[-r, 0)$ and have a discontinuity at $\theta=0$ in general. However, the following lemma says that if $\bar{\phi} \in W_{\text {loc }}^{u}(\varepsilon)$, $|\bar{\phi}| \leq \delta_{2}$, and $|\varepsilon| \leq \varepsilon_{2}$ then $\bar{g}(\bar{\phi}, \varepsilon)=0$, that is, the discontinuity will disappear on the unstable manifold.

Proposition 3.5. There exist constants $0<\delta_{3}<\delta_{2}$ and $0<\varepsilon_{3}<\varepsilon_{2}$ and a map $F: B\left(\delta_{3}\right) \times\left[-\varepsilon_{3}, \varepsilon_{3}\right] \rightarrow L\left(S, L^{\infty}\right)$ such that

$$
\bar{g}(\bar{\phi}, \varepsilon)=F(\bar{\phi}, \varepsilon) \cdot \bar{\phi}^{s}, \quad \bar{\phi} \in B\left(\delta_{3}\right),|\varepsilon| \leq \varepsilon_{3},
$$

where $\bar{\phi}^{s}=P^{s} \bar{\phi}$. Furthermore, we have

(i) $F$ is $C^{1}$;

(ii) if $\bar{\psi}^{s} \in S$, then $\left(F(\bar{\phi}, \varepsilon) \cdot \bar{\psi}^{s}\right)(\theta)$ is $C^{1}$ in $\theta \in[-r, 0)$;

(iii) there exists a constant $K_{5}$ depending on $\delta_{3}, \varepsilon_{3}$ and $K_{1}-K_{4}$ such that for every $\phi \in B\left(\delta_{3}\right),|\varepsilon| \leq \varepsilon_{3}$ and $\bar{\psi}^{s} \in S$, the following estimates hold: 


$$
\begin{gathered}
\left|F(\bar{\phi}, \varepsilon) \cdot \bar{\psi}^{s}\right|_{L^{\infty}} \leq K_{5}|\bar{\phi}|\left|\bar{\psi}^{s}\right|, \\
\sup _{-r \leq \theta<0}\left|\frac{d}{d \theta}\left(F(\bar{\phi}, \varepsilon) \cdot \bar{\psi}^{s}\right)(\theta)\right| \leq K_{5}\left|\bar{\psi}^{s}\right| .
\end{gathered}
$$

Proof. We will show first that there exist $0<\delta_{3}<\delta_{2}$ and $0<\varepsilon_{3}<\varepsilon_{2}$ such that if $|\bar{\phi}|<\delta_{3}, \bar{\phi} \in U$ and $|\varepsilon| \leq \varepsilon_{3}$, then

$$
\bar{g}(\bar{\phi}, \varepsilon)(\theta)=0, \quad \theta \in[-r, 0] \text {. }
$$

Recall that $\bar{\phi}=H(\phi, \varepsilon)$ is defined by

$$
\bar{\phi}^{s}=\phi^{s}-h_{u}\left(\phi^{u}, \varepsilon\right), \quad \bar{\phi}^{u}=\phi^{u} .
$$

Assume that $|\bar{\phi}|<\delta_{2}$ and $|\varepsilon| \leq \varepsilon_{2}$. Let

$$
t_{0}=t_{0}(\phi, \varepsilon)=\sup \left\{t \geq 0: x_{t}(\phi, \varepsilon) \in H^{-1}\left(B\left(\delta_{2}\right), \varepsilon\right)\right\} \text {. }
$$

It is not hard to see from the saddle point property that if $\bar{\delta}_{2}>0$ is sufficiently small, then $t_{0} \geq 2 r$ for all $|\phi| \leq \delta_{2}$ and $|\varepsilon| \leq \varepsilon_{2}$. Since $H$ is near the identity, there exist $0<\varepsilon_{3}<\varepsilon_{2}$ and $0<\delta_{3}<\delta_{2}$ such that

$$
B\left(\delta_{3}\right) \subset H\left(B\left(\bar{\delta}_{2}\right), \varepsilon\right), \quad|\varepsilon| \leq \varepsilon_{3} .
$$

Now, we will show (3.20) holds true for all $|\bar{\phi}| \leq \delta_{3}, \bar{\phi} \in U$ and $|\varepsilon| \leq \varepsilon_{3}$. Suppose the contrary; then there exist $\bar{\phi}_{0}^{u} \in U,\left|\bar{\phi}_{0}^{u}\right| \leq \delta_{3},\left|\varepsilon_{0}\right| \leq \varepsilon_{3}$ and $\theta_{0} \in[-r, 0]$ such that

$$
\bar{g}\left(\bar{\phi}_{0}^{u}, \varepsilon_{0}\right)\left(\theta_{0}\right) \neq 0 \text {. }
$$

Let $\phi_{0} \in H^{-1}\left(\bar{\phi}_{0}^{u}, \varepsilon_{0}\right)$. Then $\phi_{0} \in W_{\text {loc }}^{u}\left(\varepsilon_{0}\right)$ and $\left|\phi_{0}\right| \leq \bar{\delta}_{2}$. By the choice of $\delta_{2}, t_{0}=t_{0}\left(\phi_{0}, \varepsilon_{0}\right) \geq 2 r$. Hence, $\left[-\theta_{0}, t_{0}-\theta_{0}\right]$ is nonempty. Let $\bar{x}_{t}=$ $H\left(x_{t}\left(\phi_{0}, \varepsilon_{0}\right), \varepsilon_{0}\right),-\theta_{0} \leq t \leq t_{0}-\theta_{0}$. Since $W_{\mathrm{loc}}^{u}\left(\varepsilon_{0}\right) \subset\left\{\bar{\phi}: \bar{\phi}^{s}=P^{s} \bar{\phi}=0\right\}$ and $\bar{x}_{t+\theta_{0}}^{s}=0$ for $-\theta_{0} \leq t \leq t_{0}-\theta_{0}, \bar{x}_{0}=H\left(\phi_{0}, \varepsilon_{0}\right)=\bar{\phi}_{0}^{u}$. By (3.17), we have

$$
0=\bar{x}_{t+\theta_{0}}^{s}=\int_{-\theta_{0}}^{t} T(t-\alpha) \bar{g}\left(\bar{x}_{a+\theta_{0}}, \varepsilon_{0}\right) d \alpha .
$$

Let $t=-\theta_{0}+\sigma$ and $0<\sigma \leq t_{0}$ with $\sigma<-\theta_{0}$ if $\theta_{0} \neq 0$. Since the above integral is an element in $C$, by (3.5) we have

$$
\begin{aligned}
0 & =\int_{-\theta_{0}}^{-\theta_{0}+\sigma}\left[T\left(-\theta_{0}+\sigma-\alpha\right) \bar{g}\left(\bar{x}_{\alpha+\theta_{0}}, \varepsilon_{0}\right)\right]\left(\theta_{0}\right) d \alpha \\
& = \begin{cases}\int_{\theta_{0}}^{-\theta_{0}+\sigma} \bar{g}\left(\bar{x}_{\alpha+\theta_{0}}, \varepsilon_{0}\right)(\sigma-\alpha) d \alpha, & -\theta_{0}>\sigma>0, \\
\int_{0}^{\sigma} T(\sigma-\alpha)\left[\bar{g}\left(\bar{x}_{\alpha}, \varepsilon_{0}\right)(0)\right] d \alpha, & \theta_{0}=0 .\end{cases}
\end{aligned}
$$

Dividing (3.23) by $\sigma$ and letting $\sigma \rightarrow 0^{+}$, we have

$$
\begin{aligned}
0 & =\lim _{\sigma \rightarrow 0^{+}} \frac{1}{\sigma} \int_{-\theta_{0}}^{-\theta_{0}+\sigma}\left[T\left(-\theta_{0}+\sigma-\alpha\right) \bar{g}\left(\bar{x}_{\alpha+\theta_{0}}, \varepsilon_{0}\right)\right]\left(\theta_{0}\right) d \alpha \\
& =\bar{g}\left(\bar{\phi}_{0}^{u}, \varepsilon_{0}\right)\left(\theta_{0}\right)
\end{aligned}
$$


for any $-r \leq \theta_{0} \leq 0$. This contradicts (3.22) and proves (3.20). Therefore,

$$
\begin{aligned}
\bar{g}(\bar{\phi}, \varepsilon) & =\bar{g}\left(\bar{\phi}^{s}+\bar{\phi}^{u}, \varepsilon\right)-\bar{g}\left(\bar{\phi}^{u}, \varepsilon\right)=\int_{0}^{1} \frac{d}{d \alpha} \bar{g}\left(\alpha \bar{\phi}^{s}+\bar{\phi}^{u}, \varepsilon\right) d \alpha \\
& =\left(\int_{0}^{1} D_{\bar{\phi}^{s}} \bar{g}\left(\alpha \bar{\phi}^{s}+\bar{\phi}^{u}, \varepsilon\right) d \alpha\right) \cdot \bar{\phi}^{s} .
\end{aligned}
$$

Define

$$
F(\bar{\phi}, \varepsilon)=\int_{0}^{1} D_{\bar{\phi}^{s}} \bar{g}\left(\alpha \bar{\phi}^{s}+\bar{\phi}^{u}, \varepsilon\right) d \alpha
$$

Since

$$
D_{\bar{\phi}^{s}} \bar{g}\left(\alpha \bar{\phi}^{s}+\bar{\phi}^{u}, \varepsilon\right)=X_{0}^{u} \bar{f}_{1}-\left(D_{\bar{\phi}^{u}} h_{u}\right) \cdot\left(X_{0}^{u} \bar{f}_{1}\right)+X_{0} \bar{f}_{1},
$$

where $h_{u}=h_{u}\left(\bar{\phi}^{u}, \varepsilon\right)$ and $\bar{f}_{1}(\bar{\phi}, \varepsilon, \alpha)=D_{\bar{\phi}^{s}} \bar{f}\left(\alpha \bar{\phi}^{s}+\bar{\phi}^{u}, \varepsilon\right), F(\bar{\phi}, \varepsilon): B\left(\delta_{3}\right) \times$ $\left[-\varepsilon_{3}, \varepsilon_{3}\right] \rightarrow L\left[S, L^{\infty}\right]$ is $C^{1}$ and $\left[F(\bar{\phi}, \varepsilon) \cdot \bar{\psi}^{s}\right](\theta)$ is $C^{1}$ in $\theta \in[-r, 0)$ for all $\bar{\psi}^{s} \in S$. This proves (i) and (ii). Furthermore, it is not hard to see from (3.25) that $F(0, \varepsilon)=0$ for all $|\varepsilon| \leq \varepsilon_{3}$. Thus, there exists $\widetilde{K}_{5}$ depending on $\delta_{2}, \varepsilon_{3}$ and $K_{1}-K_{4}$ such that

$$
\left|F(\bar{\phi}, \varepsilon) \cdot \bar{\psi}^{s}\right| \leq \widetilde{K}_{5}|\bar{\phi}|\left|\bar{\psi}^{s}\right|, \quad|\bar{\phi}|<\delta_{3},|\varepsilon| \leq \varepsilon_{3}, \bar{\psi}^{s} \in S .
$$

Moreover, by Corollary 3.3 there exists $\widetilde{\widetilde{K}}_{5}$ depending on $\delta_{3}, \varepsilon_{3}$ and $K_{1}-K_{4}$ such that

$$
\left|\frac{d}{d \theta}\left(F(\bar{\phi}, \varepsilon) \cdot \psi^{s}\right)(\theta)\right| \leq \widetilde{\widetilde{K}}_{5}\left|\bar{\psi}^{s}\right|, \quad-r \leq \theta<0,
$$

for all $|\bar{\phi}|<\delta_{3},|\varepsilon| \leq \varepsilon_{3}$ and $\bar{\psi}^{s} \in S$. Choose $K_{5}=\max \left\{\widetilde{K}_{5}, \widetilde{\widetilde{K}}_{5}\right\}$. This proves (iii).

By (3.18), we have

$$
\begin{aligned}
T(t) \bar{g}(\bar{\phi}, \varepsilon)= & T(t) X_{0}^{s} f-\left.T(t) \frac{d}{d \tau} T(\tau) h_{u}\right|_{\tau=0^{+}} \\
& -T(t) D_{\bar{\phi}^{u}} h_{u} \cdot\left(\lambda \bar{\phi}^{u}+X_{0}^{u} \bar{f}\right),
\end{aligned}
$$

where $\bar{f}=\bar{f}(\bar{\phi}, \varepsilon)$ and $h_{u}=h_{u}\left(\bar{\phi}^{u}, \varepsilon\right)$. Since $S$ is a closed subspace, $\left(D_{\bar{\phi}^{u}} h_{u}\right)$. $\left(\lambda \bar{\phi}^{u}+X_{0}^{u} \bar{f}\right) \in S$. We also have that $T(t) X_{0}^{s} \bar{f} \in S$ for $t>r$. Therefore, by Proposition 3.4(b), (3.5) and (3.26) there exists a constant $K_{6}$ depending on $\delta_{3}, \varepsilon_{3}$ and $K_{1}-K_{5}$ such that

$$
|T(t) \bar{g}(\bar{\phi}, \varepsilon)| \leq K_{6} e^{-\mu t}|\bar{\phi}|\left|\bar{\phi}^{s}\right|, \quad t \geq 0,
$$

for all $\bar{\phi} \in B\left(\delta_{3}\right)$ and $|\varepsilon| \leq \varepsilon_{3}$.

Now, we rewrite $(3.17)$ as

$$
\begin{aligned}
& \bar{x}_{t}^{s}(\bar{\phi}, \varepsilon)=T(t) \bar{\phi}^{s}+\int_{0}^{t} T(t-\alpha) F\left(\bar{x}_{\alpha}(\bar{\phi}, \varepsilon), \varepsilon\right) \cdot \bar{x}_{\alpha}^{s}(\bar{\phi}, \varepsilon) d \alpha, \\
& \bar{x}_{t}^{u}(\bar{\phi}, \varepsilon)=e^{\lambda t} \bar{\phi}^{u}+\int_{0}^{t} e^{\lambda(t-\alpha)} X_{0}^{u} \bar{f}\left(\bar{x}_{\alpha}(\bar{\phi}, \varepsilon), \varepsilon\right) d \alpha .
\end{aligned}
$$


It follows by differentiating (3.28) with respect to $\bar{\phi}$ in $L^{\infty}$ that the derivatives $D_{\bar{\phi}} \bar{x}_{t}^{s}(\bar{\phi}, \varepsilon)$ and $D_{\bar{\phi}} \bar{x}_{t}^{u}(\bar{\phi}, \varepsilon)$ satisfy the variational equations along $\bar{x}_{t}(\bar{\phi}, \varepsilon)$ :

$$
\begin{array}{rr}
u_{t}^{s}=T(t) P^{s}+\int_{0}^{t} T(t-\alpha)\left[D_{\bar{\phi}} F\left(\bar{x}_{\alpha}(\bar{\phi}, \varepsilon), \varepsilon\right) \cdot\left(u_{\alpha}, \bar{x}_{\alpha}^{s}(\bar{\phi}, \varepsilon)\right)\right. & \left.+F\left(\bar{x}_{\alpha}(\bar{\phi}, \varepsilon), \varepsilon\right) \cdot u_{\alpha}^{s}\right] d \alpha \\
0 \leq t \leq t_{0} & 0 \leq t \leq t_{0}
\end{array}
$$

where $u_{t}=D_{\bar{\phi}} \bar{x}_{t}(\bar{\phi}, \varepsilon)$ and $\left[0, t_{0}\right]$ is a definition interval for $x(\phi, \varepsilon)$.

Note that even though $\bar{x}_{t}, \bar{x}_{t}^{u}$ and $\bar{x}_{t}^{s}$ are $C^{3}$, we cannot differentiate (3.28) more than once because $F$ is only $C^{1}$. This says that by using the change of variables (3.13) we have lost some differentiability properties. However, the special forms in (3.28) and (3.29) will be sufficient to derive similar local results as in $§ 1$.

By using (3.28), (3.29), (3.5), (3.10), (3.27), and Propositions 3.1-3.5, the proofs of the following lemmas can be adapted from those of Lemmas 1.21.6. We will therefore omit the proofs of Lemmas 3.6-3.8 and 3.10. We will present the proof of Lemma 3.9 since it is different from the case of semilinear parabolic equations in $\S 1$.

Lemma 3.6. For equation (3.2) there exists a constant $K_{7}$ depending on $\delta_{3}, \varepsilon_{3}$ and $K_{1}-K_{6}$ such that if $\bar{x}_{t}(\bar{\phi}, \varepsilon) \in B\left(\delta_{3}\right)$ for all $0 \leq t \leq t_{0}$ where $t_{0}>0$ is any constant, then

$$
\left|\bar{x}_{t}^{s}(\bar{\phi}, \varepsilon)\right| \leq K_{7}\left|\bar{\phi}^{s}\right| e^{-\mu t}, \quad 0 \leq t \leq t_{0} .
$$

Lemma 3.7. For equation (3.2) there exist $0<\delta_{4}<\delta_{3}$ and constants $\tilde{\mu}, \tilde{\lambda}, \tilde{\lambda}>0$ such that if $\bar{x}_{t}(\bar{\phi}, \varepsilon) \in B\left(\delta_{4}\right)$ for $0 \leq t \leq t_{0}$ where $t_{0}$ is any constant, then

$$
\begin{array}{lc}
\left|D_{\bar{\phi}} \bar{x}_{t}^{s}(\bar{\phi}, \varepsilon)\right| \leq 2 e^{-\tilde{\mu} t}, \quad 0 \leq t \leq t_{0}, \varepsilon \in\left[-\varepsilon_{3}, \varepsilon_{3}\right], \\
\left|D_{\bar{\phi}} \bar{x}_{t}^{u}(\bar{\phi}, \varepsilon)\right| \leq 2 e^{\bar{\lambda} t}, \quad 0 \leq t \leq t_{0}, \varepsilon \in\left[-\varepsilon_{3}, \varepsilon_{3}\right], \\
\left|D_{\bar{\phi}^{u}} \bar{x}_{t}^{u}(\bar{\phi}, \varepsilon)\right| \leq \frac{1}{2} e^{\tilde{\lambda} t}, \quad 0 \leq t \leq t_{0}, \varepsilon \in\left[-\varepsilon_{3}, \varepsilon_{3}\right] .
\end{array}
$$

Note that by the change of variables $H$ in (3.13), the local stable and unstable manifolds $W_{\text {loc }}^{s}(\varepsilon)$ and $W_{\text {loc }}^{u}(\varepsilon)$ are given by

$$
\begin{aligned}
& W_{\text {loc }}^{s}(\varepsilon)=\left\{\bar{\phi}: \bar{\phi}^{u}=\bar{h}_{s}\left(\bar{\phi}^{s}, \varepsilon\right),\left|\bar{\phi}^{s}\right|<\delta_{4}\right\}, \\
& W_{\text {loc }}^{u}(\varepsilon)=\left\{\bar{\phi}: \bar{\phi}^{s}=0,\left|\bar{\phi}^{u}\right|<\delta_{4}\right\},
\end{aligned}
$$


where $\bar{h}_{s}$ is $C^{3}, \bar{h}_{s}(0, \varepsilon)=0, \varepsilon \in\left[-\varepsilon_{3}, \varepsilon_{3}\right]$ and $D_{\bar{\phi}^{s}} \bar{h}_{s}(0,0)=0$. As in $\S 1$, for every $\varepsilon \in\left[-\varepsilon_{3}, \varepsilon_{3}\right]$ we define

$$
\begin{aligned}
& \Omega\left(\delta_{4}, \rho, \varepsilon\right)=\left\{\phi^{-}:\left|\phi^{-s}\right|<\delta_{4} / K_{7},\left|\left\langle\psi_{\lambda}, \bar{\phi}^{u}-\bar{h}_{s}\left(\bar{\phi}^{s}, \varepsilon\right)\right\rangle\right|<\rho\right\} \subset B\left(\delta_{4}\right), \\
& \Omega^{+}\left(\delta_{4}, \rho, \varepsilon\right)=\left\{\bar{\phi}: \bar{\phi} \in \Omega\left(\delta_{4}, \rho, \varepsilon\right), 0<\left\langle\psi_{\lambda}, \bar{\phi}^{u}-\bar{h}_{s}\left(\bar{\phi}^{s}, \varepsilon\right)\right\rangle<\rho\right\} \\
& \Omega^{-}\left(\delta_{4}, \rho, \varepsilon\right)=\left\{\bar{\phi}: \bar{\phi} \in \Omega\left(\delta_{4}, \rho, \varepsilon\right),-\rho<\left\langle\psi_{\lambda}, \bar{\phi}^{u}-\bar{h}_{s}\left(\bar{\phi}^{s}, \varepsilon\right)\right\rangle<0\right\},
\end{aligned}
$$

where $\langle\cdot, \cdot\rangle$ is the bilinear form given by (3.3). Note that since $W_{\text {loc }}^{s}(\varepsilon)$ has codimension one and $\Omega^{+} \cap \Omega^{-}=\varnothing, \Omega=\Omega^{+} \cup \Omega^{-} \cup W_{\text {loc }}^{s}(\varepsilon)$, where $\Omega=$ $\Omega\left(\delta_{4}, \rho, \varepsilon\right)$ and $\Omega^{ \pm}=\Omega^{ \pm}\left(\delta_{4}, \rho, \varepsilon\right)$.

Lemma 3.8. Let $\left(\bar{x}_{t}^{s}(\bar{\phi}, \varepsilon), \bar{x}_{t}^{u}(\bar{\phi}, \varepsilon)\right)$ satisfy $(3.28)$ in $B\left(\delta_{4}\right)$ and $0<\rho<\delta_{4} / 4$. If $\bar{\phi} \in \Omega^{+}\left(\delta_{4}, \rho, \varepsilon\right) \cup \Omega^{-}\left(\delta_{4}, \rho, \varepsilon\right)$, then there exists $\tau=\tau(\bar{\phi}, \varepsilon)>0$ such that

$$
\begin{aligned}
& \bar{x}_{\tau}^{u}(\bar{\phi}, \varepsilon)-\bar{x}_{\tau}^{u}\left(\bar{\phi}^{s}+\bar{h}_{s}\left(\bar{\phi}^{s}, \varepsilon\right), \varepsilon\right) \\
& \quad= \begin{cases}\delta_{4} / 2, \quad \text { if } \bar{\phi} \in \Omega^{+}\left(\delta_{4}, \rho, \varepsilon\right), & |\varepsilon| \leq \varepsilon_{3}, \\
-\delta_{4} / 2, & \text { if } \bar{\phi} \in \Omega^{-}\left(\delta_{4}, \rho, \varepsilon\right),\end{cases}
\end{aligned}
$$

Furthermore, if $\bar{\phi} \in \Omega^{+}\left(\delta_{4}, \rho, \varepsilon\right) \cup \Omega^{-}\left(\delta_{4}, \rho, \varepsilon\right)$ and $|\varepsilon| \leq \varepsilon_{3}$, then

$$
\begin{gathered}
\frac{1}{\tilde{\lambda}} \ln \frac{\delta_{4}}{4\left|\left\langle\psi_{\lambda}, \bar{\phi}^{u}-\bar{h}_{s}\left(\bar{\phi}^{s}, \varepsilon\right)\right\rangle\right|} \leq \tau \leq \frac{1}{\tilde{\lambda}} \ln \frac{\delta_{4}}{\left|\left\langle\psi_{\lambda}, \bar{\phi}^{u}-\bar{h}_{s}\left(\bar{\phi}^{s}, \varepsilon\right)\right\rangle\right|}, \\
\left|D_{\bar{\phi}} \tau(\bar{\phi}, \varepsilon)\right| \leq \frac{8}{\lambda \delta_{4}-8 K_{2} \delta_{4}^{2}} e^{\tilde{\lambda} \tau(\bar{\phi}, \varepsilon)},
\end{gathered}
$$

where $K_{2}$ is given in (3.10).

Lemma 3.9. Let $\bar{\phi} \in \Omega\left(\delta_{4}, \rho, \varepsilon\right),|\varepsilon| \leq \varepsilon_{3}$ and $t_{0}>2 r$ be as in (3.21). There exists a constant $K_{8}$ depending on $\delta_{3}, \varepsilon_{3}$ and $K_{1}-K_{7}$ such that if a solution $\left(\bar{x}_{t}^{s}(\bar{\phi}, \varepsilon), \bar{x}_{t}^{u}(\bar{\phi}, \varepsilon)\right)$ of equation (3.28) is in $B\left(\delta_{4}\right)$ for $0 \leq t \leq t_{0}$, then $\bar{x}_{t}^{s}(\bar{\phi}, \varepsilon)$ is differentiable in $t \in\left(r, t_{0}\right)$ and satisfies

$$
\left|\frac{d}{d t} \bar{x}_{t}^{s}(\bar{\phi}, \varepsilon)\right| \leq K_{8}|\bar{\phi}| e^{-\mu t}, \quad r<t<t_{0},
$$

where the derivative $\frac{d}{d t}$ is taken in $L^{\infty}$.

Proof. Let $t>r, \bar{x}_{t}=\bar{x}_{t}(\bar{\phi}, \varepsilon)$ and $\bar{x}_{t}^{s}=\bar{x}_{t}^{s}(\bar{\phi}, \varepsilon)$. By (3.28) we have

$$
\begin{aligned}
\bar{x}_{t}^{s}(\theta)= & T(t+\theta) \bar{\phi}^{s}(0)+\int_{0}^{t+\theta} T(t-\alpha+\theta)\left[F\left(\bar{x}_{\alpha}, \varepsilon\right) \cdot \bar{x}_{\alpha}^{s}\right](0) d \alpha \\
& +\int_{t+\theta}^{t}\left[F\left(\bar{x}_{\alpha}, \varepsilon\right) \cdot \bar{x}_{\alpha}^{s}\right](\theta+t-\alpha) d \alpha, \quad \theta \in[-r, 0] .
\end{aligned}
$$

Since $\left[F(\bar{\phi}, \varepsilon) \cdot \bar{\psi}^{s}\right](\theta)$ is discontinuous only at $\theta=0$ for all $\bar{\psi}^{s} \in S$, by (3.24) and (3.25) we have

$$
\int_{t+\theta}^{t}\left[F\left(\bar{x}_{\alpha}, \varepsilon\right) \cdot \bar{x}_{\alpha}^{s}\right](\theta+t-\alpha) d \alpha=\int_{t+\theta}^{t} \widetilde{F}(\alpha, \theta+t-\alpha) \cdot \bar{x}_{\alpha}^{s} d \alpha,
$$


where

$$
\tilde{F}(\alpha, \theta) \cdot \bar{\psi}^{s}=\left[F\left(\bar{x}_{\alpha}, \varepsilon\right) \cdot \bar{\psi}^{s}\right](\theta)-X_{0}(\theta) \int_{0}^{1} \bar{f}_{1}\left(\bar{x}_{\alpha}, \varepsilon, \beta\right) d \beta \cdot \bar{\psi}^{s},
$$

and $\bar{f}_{1}(\bar{\phi}, \varepsilon, \beta)$ is as in (3.25). Note that by (3.24) and (3.25), $\widetilde{F}(\alpha, \theta)$ is $C^{1}$ in $\theta \in[-r, 0]$ and by Proposition 3.5(d) we have

$$
\begin{aligned}
& \left|\frac{\partial}{\partial \theta} \widetilde{F}(\alpha, \theta) \cdot \bar{\psi}^{s}\right| \leq K_{5}\left|\bar{\psi}^{s}\right|, \quad 0 \leq \alpha \leq t_{0}, \theta \in[-r, 0], \\
& \left|\widetilde{F}(\alpha, \theta) \cdot \bar{\psi}^{s}\right| \leq K_{5}\left|\bar{\psi}^{s}\right|, \quad 0 \leq \alpha \leq t_{0}, \theta \in[-r, 0],
\end{aligned}
$$

for all $\bar{\psi}^{s} \in S$. Hence

$$
\begin{aligned}
\frac{d}{d t} \bar{x}_{t}^{s}(\theta)= & L\left(T(t+\theta) \bar{\phi}^{s}\right)+\left[F\left(\bar{x}_{t+\theta}, \varepsilon\right) \cdot \bar{x}_{t+\theta}^{s}\right](0) \\
& +\int_{0}^{t+\theta} L\left[T(t-\alpha+\theta) F\left(\bar{x}_{\alpha}, \varepsilon\right) \cdot \bar{x}_{\alpha}^{s}\right] d \alpha+\widetilde{F}(t, \theta) \cdot \bar{x}_{t}^{s} \\
& -\widetilde{F}(t+\theta, 0) \cdot \bar{x}_{t+\theta}^{s}-\int_{t+\theta}^{t} \frac{\partial}{\partial \theta} \widetilde{F}(\alpha, \theta+t-\alpha) \cdot \bar{x}_{\alpha}^{s} d \alpha .
\end{aligned}
$$

Therefore, the desired estimate follows simply from (3.5), (3.31), Proposition 3.2 and Lemma 3.6.

Define $l: B\left(\delta_{4}\right) \times\left[-\varepsilon_{3}, \varepsilon_{3}\right] \rightarrow \mathbf{R}$ by

$$
l\left(\bar{\phi}_{1}, \bar{\phi}_{2}, \varepsilon\right)=\max _{i=1,2}\left\{\left\langle\psi_{\lambda}, \bar{\phi}_{i}^{u}-\bar{h}_{s}\left(\bar{\phi}_{i}^{s}, \varepsilon\right)\right\rangle\right\},
$$

where $\langle\cdot, \cdot\rangle$ is as in (3.3). The following lemma is analogous to Lemma 1.5.

Lemma 3.10. Let $\bar{x}^{1}(\bar{\phi}, \varepsilon)=\bar{x}_{\tau(\bar{\phi}, \varepsilon)}(\bar{\phi}, \varepsilon)$, where $\bar{\phi} \in \Omega^{+}\left(\delta_{4}, \rho, \varepsilon\right), \quad \rho<\delta_{4} / 2$ and $\varepsilon \in\left[-\varepsilon_{3}, \varepsilon_{3}\right]$. There exist constants $K_{9}$ and $a>0$ depending on $\delta_{4}, \varepsilon_{3}$ and $K_{1}-K_{8}$ such that if $\bar{\phi}_{1}, \bar{\phi}_{2} \in \Omega^{+}\left(\delta_{4}, \rho, \varepsilon\right)$ and $\varepsilon \in\left[-\varepsilon_{3}, \varepsilon_{3}\right]$, then

$$
\left|\bar{x}^{1}\left(\bar{\phi}_{1}, \varepsilon\right)-\bar{x}^{1}\left(\bar{\phi}_{2}, \varepsilon\right)\right| \leq K_{9}\left[l\left(\bar{\phi}_{1}, \bar{\phi}_{2}, \varepsilon\right)\right]^{a}\left|\bar{\phi}_{1}-\bar{\phi}_{2}\right| \text {, }
$$

where $l$ is given by (3.32).

Define

$$
\begin{aligned}
& W_{+}^{u}(\varepsilon)=\left\{\phi: \text { there exist } t>0 \text { and } \psi \in W_{\text {loc }}^{u}(\varepsilon)\right. \\
& \left.\quad \text { with }\left\langle\psi_{\lambda}, \psi^{u}-h_{s}\left(\psi^{s}, \varepsilon\right)\right\rangle>0 \text { and } \phi=x_{t}(\psi, \varepsilon)\right\}, \quad|\varepsilon| \leq \varepsilon_{3}, \\
& \phi_{1}(\varepsilon)=W_{+}^{u}(\varepsilon) \cap \Sigma\left(\delta_{4} / 2, \varepsilon\right), \quad|\varepsilon| \leq \varepsilon_{3},
\end{aligned}
$$

where

$$
\Sigma(\delta, \varepsilon)=\left\{\phi:\left\langle\psi_{\lambda}, \bar{\phi}^{u}-\bar{h}_{s}\left(\bar{\phi}^{s}, \varepsilon\right)\right\rangle=\delta, \bar{\phi}=H(\phi, \varepsilon)\right\} .
$$

Let $0<\rho_{0}<\delta_{4} / 2$ be fixed. Since $H$ is near the identity map, there exist $0<\rho_{1}<\rho_{0}$ and $0<\varepsilon_{4}<\varepsilon_{3}$ such that

$$
B\left(\rho_{1}\right) \subset H^{-1}\left(\Omega\left(\delta_{4}, \rho_{0}, \varepsilon\right), \varepsilon\right), \quad|\varepsilon| \leq \varepsilon_{4} .
$$


Let $\phi_{0} \in W_{\text {loc }}^{s}(0)$ with $\left|\phi_{0}\right|<\rho_{1}$ be fixed. By the continuity property of $W_{\text {loc }}^{s}(\varepsilon)$ in $\varepsilon$, for every $0<\rho<\operatorname{dist}\left(\partial B\left(\rho_{1}\right), \phi_{0}\right)$, there exists $0<\varepsilon_{5}(\rho)<\varepsilon_{4}$ such that

$$
B\left(\phi_{0}, \rho\right) \cap W_{\text {loc }}^{s}(\varepsilon) \neq \varnothing, \quad|\varepsilon| \leq \varepsilon_{5}(\rho) .
$$

By using the above lemmas, it is not hard to see that the proofs of the following results are exactly the same as Lemma 2.1, Theorem 2.3, and Corollary 2.4, respectively. Lemma 3.11 will be needed in the next section.

Lemma 3.11. There exists a continuous map $\pi^{1}: B\left(\phi_{0}, \rho\right) \times\left[-\varepsilon_{5}(\rho), \varepsilon_{5}(\rho)\right] \rightarrow C$ satisfying

(a) If $|\varepsilon| \leq \varepsilon_{5}(\rho),\left|\phi-\phi_{0}\right|<\rho$ and $\left\langle\psi_{\lambda}, \phi^{u}-h_{s}\left(\phi^{s}, \varepsilon\right)\right\rangle>0$, then $\pi^{1}(\phi, \varepsilon)$ is the intersection point of $\Sigma\left(\delta_{4} / 2, \varepsilon\right)$ and the orbit of the solution of (3.2) with initial value $\phi$ and parameter $\varepsilon$. On the other hand, if $\left\langle\psi_{\lambda}, \phi^{s}-h_{s}\left(\phi^{s}, \varepsilon\right)\right\rangle \leq 0$, then $\pi^{1}(\phi, \varepsilon)=\phi_{1}(\varepsilon)$;

(b) $\pi^{1}(\phi, \varepsilon)$ is continuous in $(\phi, \varepsilon)$ and is Lipschitzian in $\phi$ for each fixed $\varepsilon$. Furthermore, there exist constants $a$ and $K_{10}$ depending on $\delta_{4}, \varepsilon_{4}$ and $K_{1}-K_{9}$ such that

$$
\left|\pi^{1}\left(\phi_{1}, \varepsilon\right)-\pi^{1}\left(\phi_{2}, \varepsilon\right)\right| \leq K_{10} \rho^{a}\left|\phi_{1}-\phi_{2}\right| .
$$

In the following theorem, we may assume without loss of generality that the homoclinic orbit $\Gamma_{0}=W_{+}^{u}(0)$.

Theorem 3.12. Consider the nonlinear autonomous delay equation with a real parameter $\varepsilon \in\left[-\varepsilon_{0}, \varepsilon_{0}\right], \varepsilon_{0}>0$ :

$$
\dot{x}(t)=L\left(x_{t}\right)+f\left(x_{t}, \varepsilon\right),
$$

where $L: C \rightarrow \mathbf{R}^{n}$ is a bounded linear operator and $f: C \times\left[-\varepsilon_{0}, \varepsilon_{0}\right] \rightarrow \mathbf{R}^{n}$ in $C^{3}$. Suppose (3.34) satisfies the following:

(H4) the characteristic equation $\operatorname{det} \Delta(\lambda)=0$, where $\Delta(\lambda)=\lambda I-\int_{-r}^{0} e^{\lambda \theta}[d \eta(\theta)]$, has a unique positive solution $\lambda>0$ which is simple and the real parts of the other solutions of $\Delta(\lambda)=0$ are smaller than $-\lambda$;

(H5) $f(\phi, \varepsilon)$ satisfies $f(0, \varepsilon)=0$ and $D_{\phi} f(0, \varepsilon)=0$, where $\varepsilon \in\left[-\varepsilon_{0}, \varepsilon_{0}\right]$;

(H6) at $\varepsilon=0$, equation (3.34) has a homoclinic orbit $\Gamma_{0}$ asymptotic to the equilibrium 0 .

Then there exist a neighborhood $N\left(\Gamma_{0}\right)$ of $\Gamma_{0} \cup\{0\}$ in $C$ and $0<\bar{\varepsilon}_{0}<\varepsilon_{0}$ such that $W_{+}^{u}(\varepsilon) \subset N\left(\Gamma_{0}\right)$ and $W_{+}^{u}(\varepsilon) \cap W_{\mathrm{loc}}^{s}(\varepsilon)=\varnothing$ if and only if there exists a periodic orbit in $N\left(\Gamma_{0}\right)$, where $W_{+}^{u}(\varepsilon)$ is the orbit of (3.34) through $\phi_{0}$ satisfying $\phi_{0} \in W_{\mathrm{loc}}^{u}(\varepsilon)$ and $\left\langle\psi_{\lambda}, \phi_{0}^{u}-h_{s}\left(\phi_{0}^{s}, \varepsilon\right)\right\rangle>0$. Furthermore, this periodic orbit is unique and exponentially asymptotically stable.

We will only outline the proof of this theorem because the proof is exactly the same as that of Theorem 2.3 after we have established Lemma 3.11. Let $t_{0}>0$ be the time such that $x_{t_{0}}\left(\phi_{1}(0), 0\right)=\phi_{0}$ and $\widetilde{\Gamma}_{0}=\left\{\phi: \phi=x_{t}\left(\phi_{1}(0), 0\right), 0 \leq\right.$ $\left.t \leq t_{0}\right\} \subset \Gamma_{0}$. We define a family of maps $\pi^{2}(\cdot, \varepsilon)$ from $B\left(\phi_{1}(0), \rho_{1}\right)$ into 
a neighborhood of $\phi_{0}$ by $\pi^{2}(\phi, \varepsilon)=x_{t_{0}}(\phi, \varepsilon)$. Then for sufficiently small $\varepsilon$ and $\rho_{0}$, the composition maps $\pi(\cdot, \varepsilon)=\pi^{2}\left(\pi^{1}(\cdot, \varepsilon), \varepsilon\right)$ are contractive with a uniform contractive constant less than $1 / 2$. Then, we construct a neighborhood $N\left(\Gamma_{0}\right) \subset C$ of $\Gamma_{0} \cup\{0\}$ and $0<\bar{\varepsilon}_{0}<\varepsilon_{0}$ satisfying the following properties (see Lemmas 2.4 and 2.5):

(1) $N\left(\Gamma_{0}\right)=N_{1} \cup N_{2}$, where $N_{1}$ is a neighborhood of $\{0\}$ containing $B\left(\phi_{0}, \rho_{0}\right)$ and $B\left(\phi_{1}(0), \rho_{1}\right)$, and $N_{2}$ is a neighborhood of $\widetilde{\Gamma}_{0}$;

(2) if $\gamma$ is an orbit in $C$ of equation (3.34) at $\varepsilon \in\left[-\bar{\varepsilon}_{0}, \bar{\varepsilon}_{0}\right]$ satisfying $\gamma \cap N_{1} \neq \phi$ and $\gamma \subset N\left(\Gamma_{0}\right)$, then $\left\langle\psi_{\lambda}, \phi^{u}-h_{s}\left(\phi^{u}, \varepsilon\right)\right\rangle \geq 0$ for every $\phi \in \gamma \cap N_{1}$;

(3) if $\phi \in N_{2}$ and $|\varepsilon| \leq \bar{\varepsilon}_{0}$, then there exists $t>0$ such that $x_{t}(\phi, \varepsilon) \in$ $B\left(\phi_{0}, \rho_{0}\right)$;

(4) if $\phi \in B\left(\phi_{1}(0), \rho_{1}\right)$ and $|\varepsilon| \leq \bar{\varepsilon}_{0}$, then $x_{t}(\phi, \varepsilon) \in N_{2}$ for all $0 \leq t \leq t_{0}$;

(5) $\phi_{1}(\varepsilon)$ and the fixed point $\phi_{*}(\varepsilon)$ of $\pi(\cdot, \varepsilon)$ are sufficiently close to $\phi_{1}(0)$ and $\phi_{0}$, respectively (see Lemma $2.5(\mathrm{~b}),(\mathrm{c})$ ).

After having defined $N\left(\Gamma_{0}\right)$ and $\bar{\varepsilon}_{0}$, we can show that the condition that $W_{+}^{u}(\varepsilon) \subset N\left(\Gamma_{0}\right)$ and $W_{+}^{u}(\varepsilon) \cap W_{\text {loc }}^{s}(\varepsilon)=\varnothing$ is equivalent to $\left\langle\psi_{\lambda}, \phi_{*}^{u}-h_{s}\left(\phi_{*}^{s}, \varepsilon\right)\right\rangle>$ 0 , where $\phi_{*}=\phi_{*}(\varepsilon)$ is the fixed point of $\pi(\cdot, \varepsilon)$. However, the last condition is equivalent to the existence of a unique periodic orbit of equation (3.34) in $N\left(\Gamma_{0}\right)$. The stability property of the periodic orbit follows from the contraction property of the map $\pi$.

Corollary 3.13. Suppose the same hypotheses of Theorem 3.12 hold for equation (3.34). Then there exists a neighborhood $N\left(\Gamma_{0}\right)$ of $\Gamma_{0} \cup\{0\}$ in $C$ and $0<$ $\bar{\varepsilon}_{0}<\varepsilon_{0}$ such that if there is a homoclinic orbit in $N\left(\Gamma_{0}\right)$ for equation (3.34) at $|\varepsilon| \leq \bar{\varepsilon}_{0}$, then there exist no periodic orbits in $N\left(\Gamma_{0}\right)$.

\section{Applications}

As an application, we consider the following functional differential delay equation (see [6 and 11]):

$$
\dot{x}(t)=a f(x(t-1)), \quad x \in \mathbf{R},
$$

where $a$ is a real parameter. We assume that $f: \mathbf{R} \rightarrow \mathbf{R}$ is periodic with minimal period $-A+B, A<0<B, f(A)=f(B)=0$ and $f^{\prime}(A)=1$. Note that if $x$ is a solution of (4.1), then $x+n(-A+B)$ is a solution of (4.1) for every integer $n . x \equiv A$ and $x \equiv B$ are equilibria of (4.1). A solution $x$ of (4.1) is called a heteroclinic solution from $A$ to $B$ if

$$
\lim _{t \rightarrow-\infty} x(t)=A \text { and } \lim _{t \rightarrow+\infty} x(t)=B .
$$

A solution $x$ of (4.1) is called a periodic solution of the second kind if there exists $p>0$ such that

$$
\begin{gathered}
x(t)=x(t+p)-(-A+B) \text { for all } t \in \mathbf{R}, \\
x(t) \neq x(t+q)-(-A+B) \text { for all } 0<q<p \text { and some } t \in \mathbf{R} .
\end{gathered}
$$


Note that a periodic solution of the second kind corresponds to a periodic rotation of the state variable on the circle and a heteroclinic solution from $A$ to $B$ corresponds to a homoclinic curve of the state variable on the circle (by a homoclinic curve $\Gamma(t)$ we mean $\left.\lim _{t \rightarrow-\infty} \Gamma(t)=\lim _{t \rightarrow+\infty} \Gamma(t)\right)$. We are interested in the problem of bifurcation of periodic solutions of the second kind from a heteroclinic solution from $A$ to $B$. It is obvious that our main result cannot be applied directly to this problem. However, a slight modification will be sufficient.

Theorem 4.1. Consider the functional differential delay equation with a real parameter $a \in \mathbf{R}$ :

$$
\dot{x}(t)=a f(x(t-1)) .
$$

Suppose (4.2) satisfies:

(H7) $f$ is $C^{3}$, periodic with minimal period $-A+B, A<0<B$, $B$;

(H8) at $a=a_{0}>0$ equation (4.1) has a heteroclinic solution $x^{a_{0}}$ from $A$ to

(H9) the characteristic equation $0=\lambda-a_{0} e^{-\lambda}$ has only one positive solution $\lambda_{0}$ and all other solutions $\lambda$ satisfying $\operatorname{Re} \lambda<-\lambda_{0}$.

Then there exists a neighborhood $N\left(x^{a_{0}}\right)$ in $C$ of

$$
\bigcup_{-\infty<t<\infty}\left\{x_{t}^{a_{0}}\right\} \cup\{A\} \cup\{B\}
$$

and $a_{0}^{+}>a_{0}$ such that if equation (4.1) has a periodic solution of the second kind $x$ at $a \in\left(a_{0}, a_{0}^{+}\right)$satisfying

$$
x_{t} \in \bigcup_{n \in \mathbf{Z}}\left\{N\left(x^{a_{0}}\right)+n(-A+B)\right\}, \quad t \in \mathbf{R},
$$

then it is unique and exponentially asymptotically stable.

Proof. Since solutions of $0=\lambda-a_{0} e^{-\lambda}$ are the eigenvalues of the infinitesimal generator of the strongly continuous semigroup defined by the linearized equation $\dot{x}(t)=a_{0} x(t-1)$, by (H8) and (H9), local results derived in $\S 3$ are applicable to equation (4.2) near the equilibria $A$ and $B$. Let $B(\delta)$ be a sufficiently small neighborhood of $A$ such that the local stable and unstable manifolds $W_{\mathrm{loc}}^{s}(a)$ and $W_{\mathrm{loc}}^{u}(a)$ of $A$ are defined for $a>a_{0}$ close to $a_{0}$. Since $W_{\text {loc }}^{s}(a)$ has codimension one, we may assume $B(\delta)$ is divided by $W_{\text {loc }}^{s}(a)$ into two disjoint parts $\Omega^{+}(a)$ and $\Omega^{-}(a)$ (see (3.30)). Without loss of generality, we assume $x_{t}^{a_{0}} \subset \Omega^{+}$as $t \rightarrow-\infty$. Since

$$
\lim _{t \rightarrow+\infty} x_{t}^{a_{0}}-(-A+B)=A
$$

we can choose $\phi_{0} \in x_{t}^{a_{0}}-(-A+B)$ with $\phi_{0} \in B(\delta)$. By (3.33) we can define $\phi_{1}(a) \in W_{\text {loc }}^{u}(A)$ for $a>a_{0}$ close to $a_{0}$ such that $\phi_{1}(a) \in \Omega^{+}(a)$ is continuous in $a$ with $\phi_{1}\left(a_{0}\right)=x_{t}^{a_{0}}$ for some $t<0$. By Lemma 3.11, for every small $\rho>0$, there exist $a(\rho)>0$ and a continuous map $\pi^{1}: B\left(\phi_{0}, \rho\right) \times\left[a_{0}, a(\rho)\right]$ 
satisfying (a) if $a \in\left[a_{0}, a(\rho)\right],\left|\phi-\phi_{0}\right|>\rho$ and $\phi \in \Omega^{+}(a)$, then $\pi^{1}(\phi, a)$ is on the orbit of the solution $x(\phi, a)$ of (4.2). On the other hand, if $\phi \in$ $\Omega^{-}(a)$, then $\pi^{1}(\phi, a)=\phi_{1}(a) ;(b) \pi^{1}(\phi, a)$ is Lipschitzian in $\phi$ for each fixed $a \in\left[a_{0}, a(\rho)\right]$ with a Lipschitz constant $K \rho^{6}$, where constants $K$ and $\rho$ are independent of $a \in\left[a_{0}, a(\rho)\right]$. Let $t_{0}>0$ be the time such that $x_{t_{0}}\left(\phi_{1}\left(a_{0}\right), a_{0}\right)-(-A+B)=\phi_{0}$. Then, we define a map $\pi^{2}: B\left(\phi_{1}\left(a_{0}\right), \rho_{1}\right) \times$ $\left[a_{0}, a_{1}\right] \rightarrow B(\delta)$, where $\rho_{1}$ and $a_{1}$ are some sufficiently small number, by $\pi^{2}(\phi, a)=x_{t}(\phi, a)-(-A+B)$. Therefore, if $\rho_{0}$ is sufficiently small, then the composition $\pi=\pi^{2}\left(\pi^{1}(\cdot, \cdot), \cdot\right): B\left(\phi_{0}, \rho_{0}\right) \times\left[a_{0}, a\left(\rho_{0}\right)\right] \rightarrow B\left(\phi_{0}, \rho_{0}\right)$ satisfies that for each fixed $a \in\left[a_{0}, a\left(\rho_{0}\right)\right], \pi(\cdot, a)$ is a contraction map with a uniform contractive constant less than $1 / 2$. Next, we construct a neighborhood $N\left(x_{t}^{a_{0}}\right) \subset C$ of $\left\{x_{t}^{a_{0}}: t \in \mathbf{R}\right\} \cup\{A, B\}$ and $a_{0}^{+}$satisfying

(1) $N\left(x^{a_{0}}\right)=N_{1} \cup \tilde{N}_{1} \cup N_{2}$, where $N_{1}$ is a neighborhood of $A$ containing $B\left(\phi_{0}, \rho_{0}\right)$ and $B\left(\phi_{1}\left(a_{0}\right), \rho_{1}\right), \tilde{N}_{1}=N_{1}+(-A+B)=\left\{\phi: \phi-(-A+B) \in N_{1}\right\}$ which is a neighborhood of $B$ and $N_{2}$ is a neighborhood of $\left\{x_{t}\left(\phi_{1}\left(a_{0}\right), a_{0}\right)\right.$ : $\left.0 \leq t \leq t_{0}\right\}$;

(2) if $\gamma$ is an orbit in $C$ satisfying

$$
\gamma \subset \bigcup_{u \in \mathbf{Z}}\left\{N\left(\Gamma_{0}\right)+n(-A-B)\right\}
$$

and $\gamma \cap\left\{N_{1}+n(-A+B)\right\}$ for some $n$, then $\phi-n(-A+B) \in \Omega^{+}(a) \cup W_{\mathrm{loc}}^{\mathrm{s}}(a)$ for every $\phi \in \gamma \cap\left\{N_{1}+n(-A+B)\right\}$;

(3) if $\phi \in N_{2}$ and $a \in\left[a_{0}, a_{0}^{+}\right]$, then there exists $t>0$ such that $x_{t}(\phi, a)-$ $(-A+B) \in B\left(\phi_{0}, \rho_{0}\right)$

(4) if $\phi \in B\left(\phi_{1}(0), \rho_{1}\right)$ and $a \in\left[a_{0}, a_{0}^{+}\right]$, then $x_{t}(\phi, a) \in N_{2}$ for $0 \leq t \leq t_{0}$;

(5) $\phi_{1}(a)$ and the fixed point $\phi_{*}(a)$ of $\pi(\cdot, a)$ are sufficiently close to $\phi_{1}\left(a_{0}\right)$ and $\phi_{0}$, respectively (see Lemma $2.5(\mathrm{~b}),(\mathrm{c})$ ).

By (a) and (2) and (3) of the above properties, any orbit in $C$ of a periodic solution of the second kind $x$ satisfying (4.2) must contain the fixed point $\phi_{*}(a)$ of $\pi(\cdot, a)$ and $\phi_{*}(a) \in \Omega^{+}(a)$. This proves the uniqueness. By (b) and (4) and (5) of the above properties, the periodic solution of the second kind is exponentially asymptotically stable.

The following is taken from Walther [11].

Suppose $r>0$ and $\xi_{1}, \xi_{2} \in \mathbf{R}$ satisfy

$$
A+2 r<\xi_{1}<\xi_{2}<-r<r<B-r .
$$

Let $g: \mathbf{R} \rightarrow \mathbf{R}$ be $C^{1}$ and satisfy

(i) $g$ is periodic with minimal period $-A+B$,

(ii) $g(A)=0,0<g$ in $(A, 0), g(0)=0, g<0$ in $(0, B)$,

(iii) $|g|<r / 2$ in $(A-r, A+r) \cup(-r, B-r)$,

(iv) there exists $q \in(0,1)$ with $|g(\xi)| \leq q|\xi-A|$ for all $\xi \in(A-r, A+r)$, 
(v) $a^{+}=g^{\prime}(A)$ is positive with $\log a^{+}<-\lambda\left(a^{+}\right)$, where $\lambda\left(a^{+}\right)$satisfies $\lambda\left(a^{+}\right)=a^{+} e^{-\lambda\left(a^{+}\right)}$,

(vi) for $\phi \in C, A \leq \phi \leq \xi_{1}$ and $\phi(0)=\xi_{1}$ imply $\xi_{1}+\int_{-1}^{0} g \cdot \phi(s) d s \leq \xi_{2}$, and for $\psi \in C, \xi_{1} \leq \psi \leq \xi_{2}$ and $\psi(0)=\xi_{2}$ imply $B+r<\xi_{2}+\int_{-1}^{0} g \cdot \psi(s) d s$.

Consider

$$
f(\xi)=g(\xi) / g^{\prime}(A), \quad \xi \in \mathbf{R} .
$$

Theorem 4.2. Let $f: \mathbf{R} \rightarrow \mathbf{R}$ be defined by (4.4). Suppose $f$ satisfies conditions (i)-(vi). Then

(a) there exists a nonempty interval $\left[a_{0}, a_{1}\right)$ with $a_{1}>a_{0}>0$ such that if $a=a_{0}$, then equation (4.1) has a heteroclinic solution $x^{a_{0}}$ from $A$ to $B$, and if $a \in\left(a_{0}, a_{1}\right)$, then equation (4.1) has a periodic solution of the second kind $x^{a}$. Furthermore, for every $a \in\left[a_{0}, a_{1}\right]$ there exists $\phi_{a} \in \gamma^{a}$ (the orbit in $C$ of $x^{a}$ ) such that $\phi_{a} \rightarrow \phi_{a_{0}}$ as $a \rightarrow a_{0}$;

(b) the characteristic equation $0=\lambda-a_{0} e^{-\lambda}$ has exactly one positive solution $\lambda_{0}$ and all other solutions $\lambda$ satisfy the inequality $\operatorname{Re} \lambda<-\lambda_{0}$.

By Theorems 4.1 and 4.2 above, we have the following:

Corollary 4.3. Suppose $f: \mathbf{R} \rightarrow \mathbf{R}$ is $C^{3}$ and satisfies the conditions in Lemma 4.2. Then there exists $0<a_{0}<a_{0}^{+}<a_{1}$ and a neighborhood $N\left(x^{a_{0}}\right) \subset C$ of $\left\{x_{t}^{a_{0}}: t \in \mathbf{R}\right\} \cup\{A\} \cup\{B\}$ such that if $a \in\left(a_{0}, a_{0}^{+}\right)$, then equation (4.1) has a unique and exponentially asymptotically stable periodic solution of the second kind in

$$
G=\bigcup_{n \in \mathbf{Z}}\left\{N\left(x^{a_{0}}\right)+n(-A+B)\right\} .
$$

Proof. Let $N\left(x^{a_{0}}\right)$ and $a_{0}^{+}$be defined as in Theorem 4.1. Without loss of generality, we may assume $\phi_{a}-(-A+B)=\phi_{*}(a)$, the unique fixed point of the map $\pi(\cdot, a)$ defined in Theorem 4.1 for every $a \in\left[a_{0}, a^{+}\right)$. Therefore, the orbit $\gamma^{a}$ of a periodic solution of the second kind $x^{a}$ must be in $G$ for all $a \in\left(a_{0}, a_{0}^{+}\right)$. Hence, by Theorem $4.1 x^{a}$ is unique and exponentially asymptotically stable.

Example. Let $\rho \in(\pi / 2, \pi)$ be given. Let $g_{w}(\xi)=\rho(\sin w-\sin (\xi+w))$ for $\xi \in \mathbf{R}$ and $w \in(0, \pi / 2)$. Then there exists $w_{0} \in(0, \pi / 2)$ such that for every $w \in\left(w_{0}, \pi / 2\right)$ the real numbers $A=-\pi-2 w, B=\pi-2 w, r=B / 3$, $\xi_{1}=A+w, \xi_{2}=-w$ and $g_{w}(\xi)$ satisfy hypotheses (i)-(vi) (see [6]). The corresponding equation $\dot{x}(t)=a f(x(t-1))$ with $a>0$ and $f=g_{w} / g_{w}^{\prime}(A)$ models phase-locked loops for the control of high frequency generators (see [6 and 11]).

\section{REFERENCES}

1. A. A. Andronov, E. A. Leontovich, J. I. Gordon and A. G. Maier, Theory of bifurcations of dynamical systems on a plane, Wiley, New York, 1973.

2. C. M. J. Blazqueg, Bifurcation from homoclinic orbits in parabolic equations, preprint. 
3. S.-N. Chow and J. K. Hale, Methods of bifurcation theory, Springer-Verlag, New York, 1982.

4. J. W. Evans, N. Fenichel and J. A. Feroe, Double impulse solutions in nerve axon equations, SIAM J. Appl. Math. 42 (1982), 219-234.

5. J. A. Feroe, Temporal stability of solitary impulse solutions of a nerve equation, Biophys. J. 21 (1978), 102-110.

6. T. Furomochi, Existence of periodic solutions of dimensional-delay equations, Tôhoku Math. J. 30 (1978), 13-35.

7. J. K. Hale, Functional differential equations, Springer-Verlag, New York, 1977.

8. D. Henry, Geometric theory of semilinear parabolic equations, Springer-Verlag, New York, 1981.

9. Ju. I. Neimark and L. P. Šil'nikov, A case of generation of periodic motions, Soviet Math. Dokl. 6 (1965), 1261-1264.

10. L. P. Šil' nikov, On the generation of periodic motion from trajectories doubly asymptotic to an equilibrium state of saddle type, Mat. Sb. 77 (1968), 427-438.

11. H.-O. Walther, Bifurcation from a heteroclinic solution in delay differential equations, Trans. Amer. Math. Soc. 290 (1985), 213-233.

12. __ Bifurcation from saddle connection in functional differential equations: an approach with inclination lemmas, preprint. 48824

Current address (Shui-Nee Chow): Center for Dynamical Systems, Georgia Institute of Technology, Atlanta, Georgia 30332

Current address (Bo Deng): Department of Mathematics and Statistics, University of NebraskaLincoln, Lincoln, Nebraska 68588 